Universidad Nacional de La Plata

Facultad de Ciencias Médicas

Maestría en Educación Terapéutica de personas con diabetes y otros factores de riesgo cardiovascular.

\title{
"Actividades recreativo-educativas: cambios inducidos en un grupo de niños con diabetes tipo 1 y sus familiares"
}

\section{AUTOR:}

Nora Mercuri

PADRINO DE TESIS:

Dr. Juan José Gagliardino

LUGAR DE TRABAJO:

Centro de Endocrinología Experimental y Aplicada (CENEXA)

Facultad de Ciencias Medicas (UNLP-CONICET)

Centro colaborador de la OPS-OMS

\section{FECHA:}

Noviembre 2006 


\begin{tabular}{ll} 
Resumen & 2 \\
\hline Introducción & 4 \\
\hline Objetivos & 11 \\
\hline Material y Métodos & 12 \\
\hline Área y periodo de estudio & 13 \\
\hline Población & 15 \\
\hline Programa de Actividades & 16 \\
\hline Modelo educativo & 19 \\
\hline Fuentes y Tipo de Información & 33 \\
\hline Análisis Estadistico & 38 \\
Resultados & 39 \\
\hline Discusión y Conclusiones & 52 \\
\hline Bibliografia & 61 \\
\hline Testimonios & 73 \\
\hline Anexos & 76 \\
\hline Numero 1 & 77 \\
\hline Carta de consentimiento informado & \\
\hline Numero 2 & 78 \\
\hline Programa de actividades (Dia Tipo) del Campamento 2004 & \\
\hline Numero 3 & \\
\hline Programa de actividades del Encuentro Familiar 2005 & 79 \\
\hline $\begin{array}{l}\text { Numero 4 } \\
\text { Formularios de inscripción al Campamento 2004 }\end{array}$ \\
$\begin{array}{l}\text { Numero 5 } \\
\text { Formulario de inscripción al Encuentro Familiar 2005 }\end{array}$ \\
$\begin{array}{l}\text { Numero 6 } \\
\text { Formulario de seguimiento individual }\end{array}$ \\
\hline $\begin{array}{l}\text { Numero 7 } \\
\text { Registro de evaluación de habilidades }\end{array}$ \\
\hline $\begin{array}{l}\text { Numero 8 } \\
\text { Planilla de registro }\end{array}$ \\
$\begin{array}{l}\text { Numero 9 } \\
\text { Test de conocimientos }\end{array}$ \\
$\begin{array}{l}\text { Numero 10 } \\
\text { Afiche y triptico del campamento }\end{array}$ \\
\hline
\end{tabular}




\section{RESUMEN}

Introducción: La educación terapéutica es un proceso continuo e indispensable para optimizar los resultados del tratamiento y de esta forma prevenir posibles complicaciones asociadas a la diabetes. Los campamentos para niños con diabetes representan una experiencia de vida en donde las posibilidades de enseñanza-aprendizaje son favorecidas ya que se desarrollan en un ambiente distendido, seguro y en compañía de otros niños y adultos que comparten la misma afección. Las vivencias del campamento ayudan al niño a desarrollar independencia, autoconfianza y una actitud responsable y participativa dentro de su tratamiento.

El programa de educación terapéutica debe incluir a otras personas del entorno del niño, especialmente al adulto familiar, ya que la responsabilidad del autocuidado diario debe ser compartida hasta la adolescencia.

El éxito de la educación puede verse limitado por factores psicosociales que juegan un importante rol en el control de la diabetes.

Objetivo: Evaluar el efecto de un programa educativo desarrollado en un Campamento para niños con diabetes (CND) y en un Encuentro familiar (EF) sobre la incorporación de conocimientos, desarrollo de habilidades e indicadores clínico- metabólicos y terapéuticos. Metodología: El programa del CND de 7 días y del EF de 2 días, incluyó actividades deportivas y recreativas; los contenidos educativos se desarrollaron empleando estrategias individuales y grupales tales como juegos de dramatización y resolución de problemas. La preparación y autoaplicación de insulina y el automonitoreo glucémico/glucosúrico se realizaron bajo supervisión. Asistieron 37 niños con diabetes tipo 1 (de 7 a 13 años), de los cuales 19 niños y 19 familiares adultos 
participaron del EF, relevándose información las semanas anterior, durante y posterior al campamento, el primer día (pre campamento) y último día (campamento) y a los 4 y 7 meses después (post campamento). El análisis estadístico de los datos se realizó empleando el test de t, el de diferencia de proporciones, test de Tukey, y para más de 2 promedios ANOVA (IC, 95\%). Resultados: Conocimientos sobre DM (\% respuestas correctas): pre campamento $80 \pm 14 \%$; campamento $91 \pm 11 \%$ y post campamento (4 meses) $90 \pm 10 \%(p<0.001) . N^{\circ}$ de Habilidades (total 4): pre campamento $3 \pm 1$; campamento $4 \pm 1$ y post campamento (4 meses) $4 \pm 1$ ( $p<0.001)$. Control metabólico: en el período pre campamento no hubo diferencias significativas en los valores de $\mathrm{HbA} 1 \mathrm{c}$ entre niños con y sin cobertura; sus valores disminuyeron significativamente a los 4 y 7 meses: $10.3 \pm 2.3 \%$ vs. $8.8 \pm 1.8 \%$ y $9.5 \pm 2.5 \%, p<0.001)$. Los mayores descensos de $\mathrm{HbA} 1 \mathrm{c}$ se registraron en los niños con cobertura de salud que participaron del CND y del EF a los 4 y 7 meses post campamento: $9.1 \pm 1.9 \%$ vs. $7.4 \pm 0.9 \% \quad(p<0.003)$ y $7.2 \pm 1 \%$ ( $p$ $<0.002)$. En los niños sin cobertura no se registraron cambios.

Conclusión: Estos resultados muestran que la estrategia educativa implementada en el ámbito recreativo del campamento, aumentó conocimientos y promovió el desarrollo de habilidades para el autocontrol de la enfermedad. La falta 0 deficiente cobertura de salud y el status socioeconómico condicionan el beneficio de la educación terapéutica sobre el control y tratamiento de la enfermedad. 
INTRRODUCCIÓN 
La diabetes mellitus es una enfermedad metabólica caracterizada por la presencia de hiperglucemia resultante de un defecto en la secreción de insulina, en la acción de esta hormona o de la combinación de ambas (1). En la diabetes la hiperglucemia crónica está asociada a disfunción y lesiones tardías de diversos órganos, especialmente ojos, riñones, nervios, corazón y vasos sanguíneos.

La gran mayoría de los casos de diabetes se encuentran comprendidas en dos categorías: diabetes tipo 1 y diabetes tipo 2. La primera suele comenzar de manera súbita en la primera infancia o en la adolescencia y es causada por la destrucción autoinmune de las células $\beta$ del páncreas que genera un déficit absoluto de insulina; por esta razón estas personas requieren insulina para sobrevivir. La diabetes tipo 2 suele comenzar en personas mayores de 40 años y su causa es la combinación de una menor sensibilidad de los tejidos a la acción de la insulina (insulinorresistencia) y una inadecuada respuesta compensatoria de su secreción. Esta última forma es el tipo más frecuente de diabetes ( 90 a $95 \%$ del total de personas con diabetes), mientras que la diabetes tipo 1 representa el 5-10\% restante. En nuestro país la prevalencia de ésta última está alrededor del 0.7 al $1.0 \%$ de personas menores de 20 años. Debe recordarse sin embargo que tanto la diabetes tipo 1 como la 2 pueden manifestarse a cualquier edad (1).

Si bien la fisiopatología y el tratamiento de las dos formas principales de diabetes es diferente, el desarrollo de complicaciones micro y macrovasculares es común a ambos tipos. El desarrollo y progresión de estas complicaciones aumenta el costo de atención de la diabetes y disminuyen la calidad de vida de quienes la padecen (2-5). La gravedad de estas 
complicaciones tardías guarda una relación directa con el grado de control glucémico y los años de evolución de la enfermedad $(6,7)$. Sin embargo, en función de la evidencia disponible se puede afirmar que el control estricto de la glucemia y de otros factores de riesgo cardiovascular asociados a la diabetes como la hipertensión arterial, reducen la frecuencia y gravedad de este tipo de complicaciones (8-10).

Por lo tanto, la calidad de vida de las personas con diabetes depende, al menos en parte, de la implementación de un programa terapéutico que apunte a optimizar el control metabólico del paciente y a prevenir el desarrollo y progresión de complicaciones agudas y crónicas de la enfermedad $(5,8-10)$.

Si bien el diagnóstico precoz es parte importante en la prevención de las complicaciones crónicas de la diabetes, la efectividad del tratamiento preventivo depende en gran medida del conocimiento, las habilidades, las actitudes y la motivación del paciente (11-14). En consecuencia el éxito de un programa terapéutico se relaciona directamente con el grado de participación activa del paciente en el control y tratamiento de su enfermedad, la que es facilitada por un proceso continuo de enseñanza-aprendizaje (15-24).

La persona con diabetes debe prestar atención a múltiples tareas relacionadas con el manejo y control de su enfermedad. Estas incluyen el monitoreo de la glucosa plasmática, la interpretación de sus resultados, los ajustes en las dosis de insulina y la medicación vía oral, el cumplimiento de un plan de alimentación adecuado, la práctica regular de actividad física y la introducción de cambios apropiados cuando no se obtienen las metas terapéuticas esperadas. Este conjunto de actividades es marcadamente 
afectado por factores psicosociales que juegan un papel importante en el control de la diabetes (25-27).

Al igual que en todas las formas clínicas de la enfermedad, la educación terapéutica de los niños con diabetes debe comenzar al momento del diagnóstico, incluyendo en ella a su entorno, principalmente su grupo familiar. Esto último es importante ya que en este grupo etáreo las decisiones y responsabilidades del autocuidado son necesariamente compartidas hasta que el niño alcanza la maduración necesaria para involucrarse con los diferentes elementos de su autocuidado (28-31). También es importante incorporar en el proceso educativo, a quienes comparten algunas actividades diarias del niño, como docentes y amigos, especialmente en lo referido a la aparición, prevención y control de eventos tales como una hipoglucemia, para que actúen adecuadamente en cada situación (32).

El logro de los objetivos de la educación terapéutica puede verse limitado por factores psicológicos (depresión, ansiedad y baja autoestima), el entorno familiar y social (estructura familiar, nivel de educación y socioeconómico, hábitos alimentarios y culturales, grado de cuidado, afecto y comprensión que la familia ofrece al paciente) y el acceso a una cobertura de salud apropiada (33-39). Por ello se considera actualmente que la identificación de problemas psicosociales y la implementación de intervenciones para solucionarlos, pueden mejorar la calidad de los resultados de un programa terapéutico y por ende la calidad de vida de la persona con diabetes $(25,27,39)$.

El objetivo común entre los familiares y el equipo de salud, debería orientarse de tal modo que los niños y jóvenes con diabetes, asuman gradualmente la responsabilidad del control y tratamiento de su enfermedad. Si 
bien la edad cronológica es un condicionante importante, no es suficiente para determinar si un niño o un joven están preparados para asumir dicha responsabilidad. Su habilidad para participar en su autocuidado varía de acuerdo a su desarrollo cognitivo, motor y a su madurez emocional (40-42). Igualmente diversos estudios han demostrado que para lograr la adherencia a las rigurosas y sistemáticas demandas del tratamiento se requiere la participación del familiar adulto aún durante la adolescencia $(43,44)$. Por lo tanto, la información provista, los métodos de enseñanza-aprendizaje y los materiales utilizados deben adaptarse a la edad y madurez del niño y a las características de su entorno familiar y social $(28,29,45)$.

Dentro de las estrategias empleadas para educar niños y adolescentes con diabetes se encuentran los campamentos recreativo/educativos. Su utilización data del año 1925, sólo 4 años después del descubrimiento de la insulina, en que se implementó en Michigan (USA), el primer campamento para niños con diabetes. Los campamentos de este tipo, con diferentes modalidades, han adquirido una importancia creciente en todo el mundo (46-60), estimándose que actualmente asisten a ellos más de 20.000 participantes con diabetes.

Los objetivos de los campamentos especializados para niños y jóvenes con diabetes apuntan a:

- Ayudar al niño a desarrollar una independencia progresiva y autoconfianza necesarias para asumir un papel activo en el control y tratamiento de su enfermedad.

- Familiarizarlo con los componentes de su control y tratamiento específico en un ambiente seguro en el que se promueve la 
incorporación de hábitos saludables (plan de alimentación y práctica regular de actividad física).

Los campamentos para niños con diabetes constituyen una alternativa educativa muy particular, ya que durante su desarrollo el proceso de enseñanza-aprendizaje es facilitado por un ambiente lúdico, la presencia de otros niños y de personal con diabetes, y una atmósfera motivadora y segura de colaboración mutua $(45,61)$.

Para los niños y jóvenes con diabetes, el campamento representa asimismo, la rara oportunidad de ser mayoría y la norma, mientras que el resto del año él es la excepción ya que convive entre pares que no comparten su misma experiencia de vivir con diabetes.

Los campamentos también constituyen una alternativa de capacitación importante para los integrantes del equipo de salud. Al compartir días enteros con sus pacientes en una atmósfera amigable y cooperativa, cada miembro del equipo, puede apreciar más claramente el efecto de sus indicaciones y consejos terapéuticos y favorecer el establecimiento de la empatía necesaria para relacionarse con ellos y su entorno. También brindan a los niños y a sus familiares una oportunidad de integración e intercambio de la experiencia de convivir con una enfermedad crónica.

Si bien durante el campamento las sesiones de educación diabetológica forman parte de los programas de actividades diarias, los campamentos están lejos de ser "clínicas en el bosque". El proceso educativo se desarrolla en forma permanente como resultado de la observación, la práctica y el intercambio de opiniones y experiencias. Asimismo, el empleo de métodos didácticos creativos e innovadores tales como la utilización de juegos 
grupales para transmitir y afianzar conceptos y habilidades relacionadas con el control de la enfermedad, estimulan al niño y favorecen su concentración mucho más que la pasividad del modelo educativo unidireccional $(13,62-65)$.

Diversos estudios demostraron el efecto positivo de los campamentos para niños y adolescentes con diabetes sobre sus conocimientos de la enfermedad, sus habilidades para el manejo de técnicas de autoevaluación y de autocuidado, su control metabólico y su autoestima $(50,51,54,57,58)$. Sin embargo, son escasos los estudios que han verificado la persistencia de los logros adquiridos durante el campamento una vez finalizado el mismo $(46,48,52,56,59,60)$. En consecuencia surgen interrogantes tales como:

¿En qué medida es posible sostener en la vida cotidiana los logros adquiridos por los niños durante el campamento?

¿Qué impacto tendría sobre dichos logros desarrollar una experiencia recreativo-educativa similar pero con la participación de sus familiares?

¿Cómo identificar quienes requieren estrategias educativas individuales además de las grupales?

El presente estudio pretende responder algunos de estos interrogantes. 
El objetivo general del estudio fue evaluar:

- en los niños asistentes al campamento: el efecto de las actividades intra y post campamento sobre sus conocimientos y habilidades y sobre indicadores clínicos, metabólicos y terapéuticos.

- en los familiares de los niños asistentes: el efecto de la aplicación de la actividad post campamento sobre sus conocimientos referidos al control y tratamiento de la diabetes.

El objetivo específico fue verificar en los niños antes, durante y después del campamento los cambios registrados en:

- peso, talla e índice de masa corporal

- sitios de inyección de insulina, número de inyecciones, tipo y dosis/día de insulina

- glucemia de automonitoreo (frecuencia y valores)

- hemoglobina glicosilada 
El modelo educativo empleado implicó el desarrollo de un Campamento para niños con diabetes tipo 1 (CND) y un Encuentro Familiar $(E F)$, con la asistencia de familiares y niños, ambos de carácter recreativoeducativo, en los cuales:

- Se emplearon estrategias educativas grupales e individuales para incrementar en los asistentes sus conocimientos, habilidades y motivación, y su utilización en la resolución de situaciones particulares relacionadas con el control de su enfermedad.

- Se brindaron conocimientos sobre la diabetes y su tratamiento en las siguientes áreas: definición, valores de referencia, prevención y desarrollo de complicaciones agudas y crónicas, automonitoreo glucémico y glucosúrico, insulinoterapia, actividad física y plan de alimentación.

- Se promovió la incorporación de hábitos saludables (plan de alimentación y práctica regular de actividad física), realización correcta de las técnicas de automonitoreo glucémico y glucosúrico, preparación y autoinyección de insulina.

\section{Área y período de estudio}

El CND, denominado "17\% Campamento Anual para niños con diabetes", se desarrolló durante 7 días en la localidad de Aguas Verdes, Municipio Urbano de la Costa, Provincia de Buenos Aires, entre el 12 y el 18 de Diciembre de 2004. El predio donde se desarrollaron las actividades cuenta con instalaciones tales como: vivienda con capacidad para albergar 80 personas, 
habitaciones para 8 personas, enfermería, comedor, cocina, lugares de esparcimiento cubiertos y al aire libre, instalaciones deportivas y un centro médico cercano para la derivación de eventuales emergencias. Se contó asimismo, con un equipo de salud y medicación para primeros auxilios y tratamiento de intercurrencias tales como alergia, asma, vómitos, diarrea, traumas menores, infecciones habituales y complicaciones agudas relacionadas con la diabetes.

El EF, denominado "III Encuentro Recreativo y Educativo para niños con diabetes y sus familiares", se desarrolló durante un fin de semana (16 y 17 de Abril de 2005), en el Camping de la Unión del Personal Civil de la Nación (UPCN), calles 89 y 143 de la Ciudad de La Plata, Provincia de Buenos Aires.

Ambas actividades se llevaron a cabo en el marco del programa de educación terapéutica para personas con diabetes y sus familiares que desarrolla el CENEXA -Centro de Endocrinología Experimental y Aplicada(UNLP-CONICET), Centro colaborador de la OPS-OMS, a través de sus convenios con la Dirección General de Cultura y Educación de la Provincia de Buenos Aires y con la Comisión de Investigaciones Científicas de la Provincia de Buenos Aires. EI CENEXA organiza en forma ininterrumpida campamentos de este tipo desde el año 1988 y encuentros familiares desde 2002, con la colaboración del Servicio de Endocrinología del Hospital de Niños "Sor María Ludovica" (La Plata), perteneciente al Ministerio de Salud de la Provincia de Buenos Aires. 


\section{Población}

La población del estudio incluyó 37 niños de ambos sexos con diabetes tipo 1 , con edades entre 7 y 13 años que cursaban el nivel de educación general básica (EGB), tuvieran o no cobertura de obra social o prepaga, y que asistieron por primera o segunda vez a un campamento organizado por el CENEXA. Se excluyó del estudio a los niños que asistieron a 2 o más campamentos. Asimismo, el estudio incluyó durante el EF a 19 familiares adultos y 19 niños que participaron del CND.

Para participar del estudio, cada familiar o tutor del niño/a inscripto al CND y el adulto participante del EF, firmaron un consentimiento informado (Anexo 1). En ningún caso se limitó la inscripción por razones económicas, disponiéndose de subsidios y becas provenientes de distintas entidades públicas y privadas, nacionales e internacionales (ver agradecimientos).

Para el desarrollo de las actividades del CND y el EF, el CENEXA contrató un Seguro de Responsabilidad Civil (Federación Patronal Seguros S.A.) para los niños y adultos participantes y los miembros del equipo interdisciplinario.

La organización, desarrollo y supervisión del CND y del EF estuvieron a cargo de un equipo interdisciplinario integrado por dos médicos pediatras especialistas en diabetes, una nutricionista, una educadora, dos profesores en educación física, 4 líderes varones y 4 líderes mujeres ( 1 líder cada 5 niños/as) y 3 personas encargadas de la cocina y de la limpieza. El líder o consejero es un/a joven con diabetes mayor de 16 años, que ha participado como campista en no menos de dos de los campamentos organizados por el 
CENEXA y que actuó previamente como ayudante de líder durante dos campamentos.

Exceptuando a los encargados de cocina y limpieza, el resto del personal del CND y el EF son integrantes habituales del equipo interdisciplinario que participa en su organización y desarrollo desde el año 2000. Todos ellos poseen amplia experiencia en campamentos de este tipo.

\section{Programa de actividades}

El programa diario del CND y EF (Anexos 2 y 3), constó de actividades atlético-deportivas, recreativas, estético-expresivas, veladas y fogones y actividades educativas relacionadas con el control y tratamiento de la diabetes. También incluyó 4 comidas (desayuno, almuerzo, merienda y cena) y 2 colaciones (mañana y tarde). Se agregó una tercera colación antes de acostarse de acuerdo al valor de glucemia y a la intensidad y duración de la actividad física desarrollada durante la noche.

El diseño del menú del CND y EF estuvo a cargo de la nutricionista. El valor calórico y la distribución de nutrientes se determinó de acuerdo a la edad, talla, peso y sexo de los niños según las recomendaciones de la Sociedad Argentina de Pediatría (66) y a la actividad física programada y desarrollada por cada niño.

Durante el CND y el EF se determinaron 4 glucemias (sangre capilar) diarias en cada niño (antes del desayuno, almuerzo, merienda y cena), utilizando tiras reactivas y glucómetro digitales. Las determinaciones de glucemia se realizaron en pequeños grupos de 4 a 6 niños, con la supervisión 
de 2 líderes. En caso de síntomas o sospecha de hipoglucemia en cualquier momento del día, se realizaron determinaciones adicionales de glucemia por el mismo método. Cada vez que la glucemia fue $\geq 250 \mathrm{mg} / \mathrm{dl}$ se realizaron determinaciones de glucosuria y cetonuria utilizando tiras de comparación visual (Keto-Diastix Bayer), bajo la supervisión de un líder.

Durante la semana del CND y el EF (antes de un almuerzo y de una merienda), se midió la glucemia a todos los niños empleando simultáneamente el método de comparación visual (Glucostix Bayer) y el glucómetro, registrándose solo el valor de éste último. Este procedimiento se repitió en otras dos oportunidades durante el CND, con el grupo de niños que utilizaba el método de comparación visual en su automonitoreo habitual. El objetivo de estas sesiones fue corregir errores de procedimiento, lectura e interpretación de resultados, enseñanza y práctica del corte longitudinal de la tira en dos partes.

La obtención de sangre para determinar la glucemia (método de comparación visual o instrumental), se realizó utilizando dispositivos para punción y lancetas individuales. En la enfermería y en los sitios destinados para las determinaciones de glucemia se colocaron contenedores para descartar el material utilizado (jeringas, agujas, lancetas y tiras reactivas).

En cada habitación, salas de determinación de glucemia y enfermería, se expuso un protocolo para el tratamiento de hipoglucemias leves a moderadas (descenso de la glucemia por debajo de valores normales que puede tratarse mediante administración de glucosa por boca). Cada líder contó en todo momento con un equipo básico para el tratamiento de hipoglucemias leves a moderadas, dentro y fuera de las instalaciones. Cada integrante del 
equipo interdisciplinario fue entrenado previamente para inyectar glucagón en caso de hipoglucemia severa (descenso de la glucemia por debajo de valores normales que requiere la asistencia de otra persona para administrar glucagón o glucosa intravenosa).

Durante el CND y el EF no se modificó el esquema de insulinoterapia registrado al ingreso del niño a esa actividad respecto al horario de aplicación y al tipo de insulina. Sin embargo, la dosis y el número de inyecciones diarias se modificó en la mayoría de los niños de acuerdo al valor de la glucemia previa a la ingesta, el consumo estimado de carbohidratos de la misma y la duración e intensidad de la actividad física previa o posterior a la comida. Los ajustes en la dosis de insulina o cantidad de carbohidratos se decidieron en forma conjunta entre el niño, el familiar adulto y el equipo organizador. Al finalizar el CND, se recomendó a los familiares y a los niños, incrementar la dosis de insulina hasta la dosis habitual (registrada al ingreso), dentro de la 24-48 horas siguientes. No hubo recomendaciones en cuanto a la frecuencia óptima de autodeterminaciones de glucemia una vez finalizado el CND y el EF.

La actividad física se distribuyó a lo largo del día en cuatro bloques o sesiones de 1 a 2 horas de duración cada uno y fue precedida por un período de 45 a 60 minutos de actividades pasivas, antes de realizar la siguiente determinación de la glucemia. Cada día se realizaron las siguientes actividades: juegos grupales de búsqueda y persecución, juegos de iniciación deportiva y deportes, caminatas, juegos nocturnos o de salón, juegos de playa y en el mar. La intensidad y duración de la actividad física se incrementó 
gradualmente desde el primer al cuarto día de campamento y descendió del quinto al séptimo día.

Desde el primer día del CND se conformaron equipos de niños teniendo en cuenta la edad (7 a 13 años), la condición y habilidades para la práctica de actividad física (según datos obtenidos al momento de la inscripción). A lo largo del CND, cada equipo acumuló puntos de acuerdo al resultado obtenido en diferentes actividades deportivas, recreativas y educativas.

\section{Modelo educativo}

El objetivo de las sesiones de educación formal del CND y el EF fue que el niño y el familiar adulto adquirieran, afianzaran y relacionaran conocimientos, resolviendo diversas situaciones problema, y que, particularmente el niño, corrigiera errores o verificara la realización correcta de diferentes técnicas empleadas en su tratamiento.

Los contenidos educativos fueron:

- Importancia del control metabólico.

- Selección y conteo de carbohidratos.

- Técnica de automonitoreo glucémico y glucosúrico, registro e interpretación de resultados.

- Efecto inmediato y a largo plazo del ejercicio sobre la glucemia.

- Técnica de preparación y autoinyección de insulina.

- Ajuste de la dosis de insulina según nivel de glucemia, ingesta estimada de carbohidratos y actividad física a desarrollar. 
- Reconocimiento y manejo de episodios de hipoglucemia, hiperglucemia y cetoacidosis.

- Complicaciones tardías de la diabetes: desarrollo, progresión y prevención

Las sesiones de educación terapéutica se desarrollaron en cinco de los siete días del CND durante la mañana y en los dos días de duración del EF durante la mañana y la tarde. Las sesiones estuvieron a cargo de los médicos, nutricionista, educadora y líderes.

Durante el CND se brindaron sesiones educativas a aquellos niños que las requerían por los siguientes motivos:

a) utilizar tiras reactivas de comparación visual para su automonitoreo glucémico habitual.

b) limitada posibilidad de emplear un plan de alimentación balanceado debido a su situación socioeconómica.

c) presencia de patología asociada como enfermedad celíaca.

En todos estos casos los niños recibieron dos sesiones educativas individuales o en pequeños grupos coordinadas por algún miembro del equipo interdisciplinario y además participaron de las actividades educativas generales.

Las técnicas educativas empleadas consistieron en resolución de problemas y dramatización, implementadas a través de juegos didácticos divididos en cinco estaciones (pequeños grupos de 7-8 niños o adultos), según sus contenidos educativos y supervisados por un coordinador. Durante el desarrollo del juego educativo, los resultados fueron evaluados y discutidos por 
los miembros del equipo que participaron como coordinadores, los niños y sus familiares.

Los grupos de niños de cada estación pertenecían a un mismo equipo; distinto a lo ocurrido en el caso de los juegos deportivos o recreativos, en los juegos educativos el puntaje otorgado dependió del grado de participación y motivación del equipo y no del resultado o la velocidad de su resolución.

Durante el EF, los adultos participaron en los mismos juegos empleados durante el CND, mientras que los niños reforzaron conceptos y aclararon dudas relacionadas al plan de alimentación. La última sesión educativa, cuyo contenido apuntaba a la integración de conocimientos, fue compartida entre los niños y sus familiares.

A continuación se describen los juegos utilizados, diseñados especialmente por el equipo interdisciplinario para el CND, según el orden en que se llevaron a cabo: 


\section{TEMA: INSULINOTERAPIA}
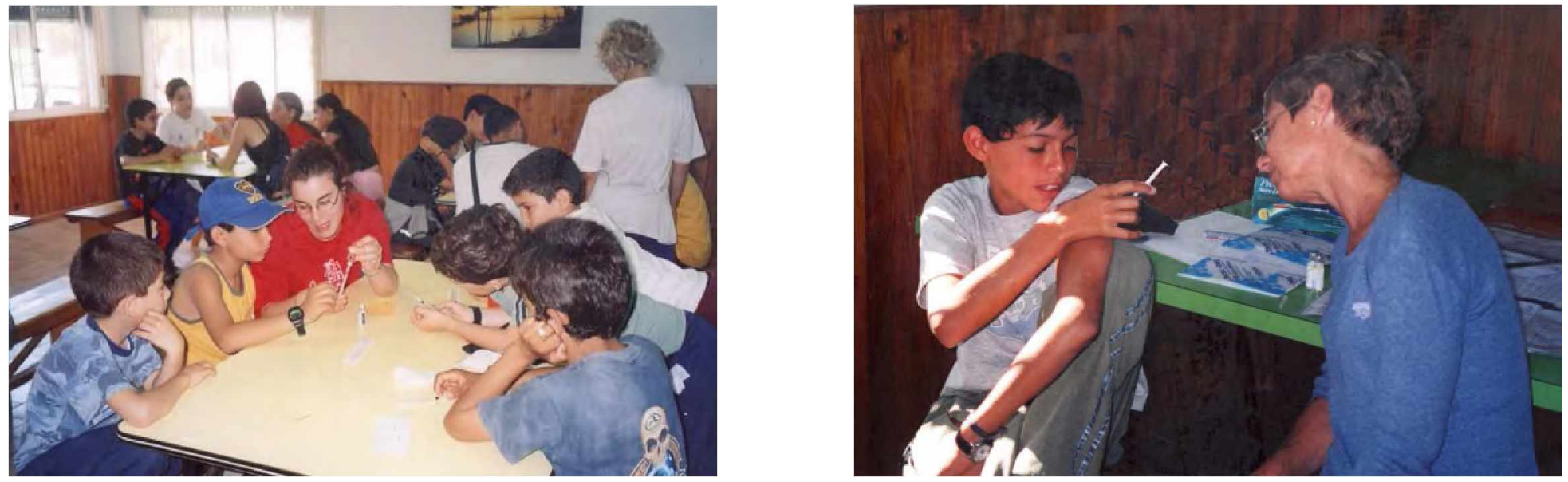

\begin{tabular}{|c|c|c|c|}
\hline CONTENIDOS & OBJETIVOS & MATERIALES & DESCRIPCION \\
\hline
\end{tabular}




\begin{tabular}{|c|c|c|c|}
\hline $\begin{array}{l}\text { Inyección de insulina y } \\
\text { rotación de los sitios de } \\
\text { inyección }\end{array}$ & $\begin{array}{l}\text { Reconocer la importancia de rotar las zonas } \\
\text { de inyección en cada aplicación. }\end{array}$ & $\begin{array}{l}\text { Mesa y sillas } \\
\text { Muñecos de } 50 \mathrm{~cm} \\
\text { de alto, forrados } \\
\text { en tela y rellenos de } \\
\text { material blando }\end{array}$ & 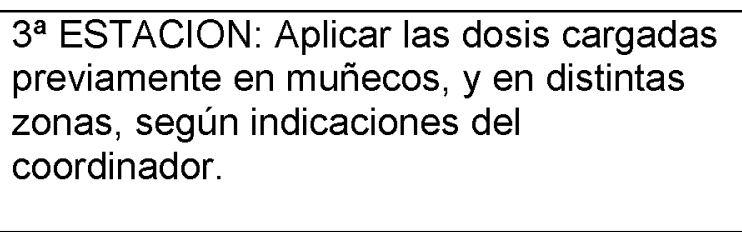 \\
\hline $\begin{array}{l}\text { Ajuste de la dosis de } \\
\text { insulina }\end{array}$ & $\begin{array}{l}\text { Reconocer cuándo y cómo se debe } \\
\text { variar la dosis de insulina. }\end{array}$ & $\begin{array}{l}\text { Cartas } \\
\text { Carteles }\end{array}$ & $\begin{array}{l}5^{a} \text { ESTACION: En un extremo de la mesa } \\
\text { se coloca un mazo de cartas con } \\
\text { situaciones problema referidas a cuándo y } \\
\text { cómo variar las dosis de insulina de } \\
\text { acción prolongada y rápida. En el otro } \\
\text { extremo se colocan encolumnadas las } \\
\text { palabras: } \\
\text { Bajar dosis: Prolongada, Rápida } \\
\text { Subir Dosis: Prolongada, Rápida } \\
\text { No Modificar } \\
\text { A su turno cada niño toma una carta, lee } \\
\text { la situación problema en voz alta y la } \\
\text { coloca debajo de la columna que } \\
\text { considera correcta. }\end{array}$ \\
\hline
\end{tabular}

Al finalizar cada estación el equipo de niños rotó a la siguiente.

El juego fue precedido por una explicación y demostración a cargo de la educadora (15 minutos de duración), acerca de las técnicas correctas de preparación y autoinyección de insulina. 
TEMA: LA IMPORTANCIA DEL CONTROL METABOLICO
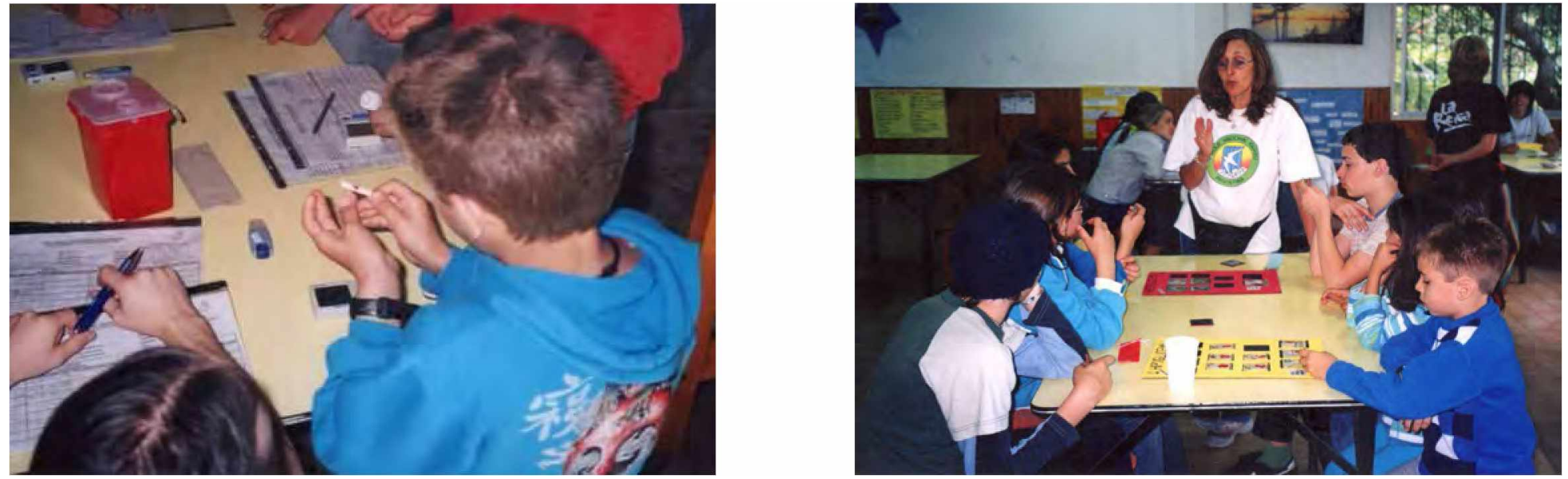

\begin{tabular}{|l|l|l|l|}
\hline \multicolumn{1}{|c|}{ CONTENIDOS } & OBJETIVOS & MATERIALES & DESCRIPCION \\
\hline $\begin{array}{l}\text { Reconocimiento y manejo } \\
\text { de los episodios de } \\
\text { hipoglucemia e } \\
\text { hiperglucemia }\end{array}$ & $\begin{array}{l}\text { Reconocer los síntomas de hipoglucemia e } \\
\text { hiperglucemia. }\end{array}$ & $\begin{array}{l}\text { Mesas y sillas } \\
\text { Tarjetas }\end{array}$ & $\begin{array}{l}\text { 1a ESTACION: A cada niño se le } \\
\text { entrega una tarjeta con una imagen } \\
\text { que representa un síntoma de } \\
\text { hipoglucemia o hiperglucemia. Por } \\
\text { turno los niños deben agrupar sobre la } \\
\text { mesa en 2 columnas las tarjetas } \\
\text { según corresponda. Luego, por turno, } \\
\text { toman al azar una tarjeta de un mazo, } \\
\text { en la que se describe una alternativa } \\
\text { de tratamiento de la hipo o } \\
\text { hiperglucemia. Luego de leerla en voz } \\
\text { alta decide al lado de qué columna } \\
\text { colocar la tarjeta. }\end{array}$ \\
\hline
\end{tabular}




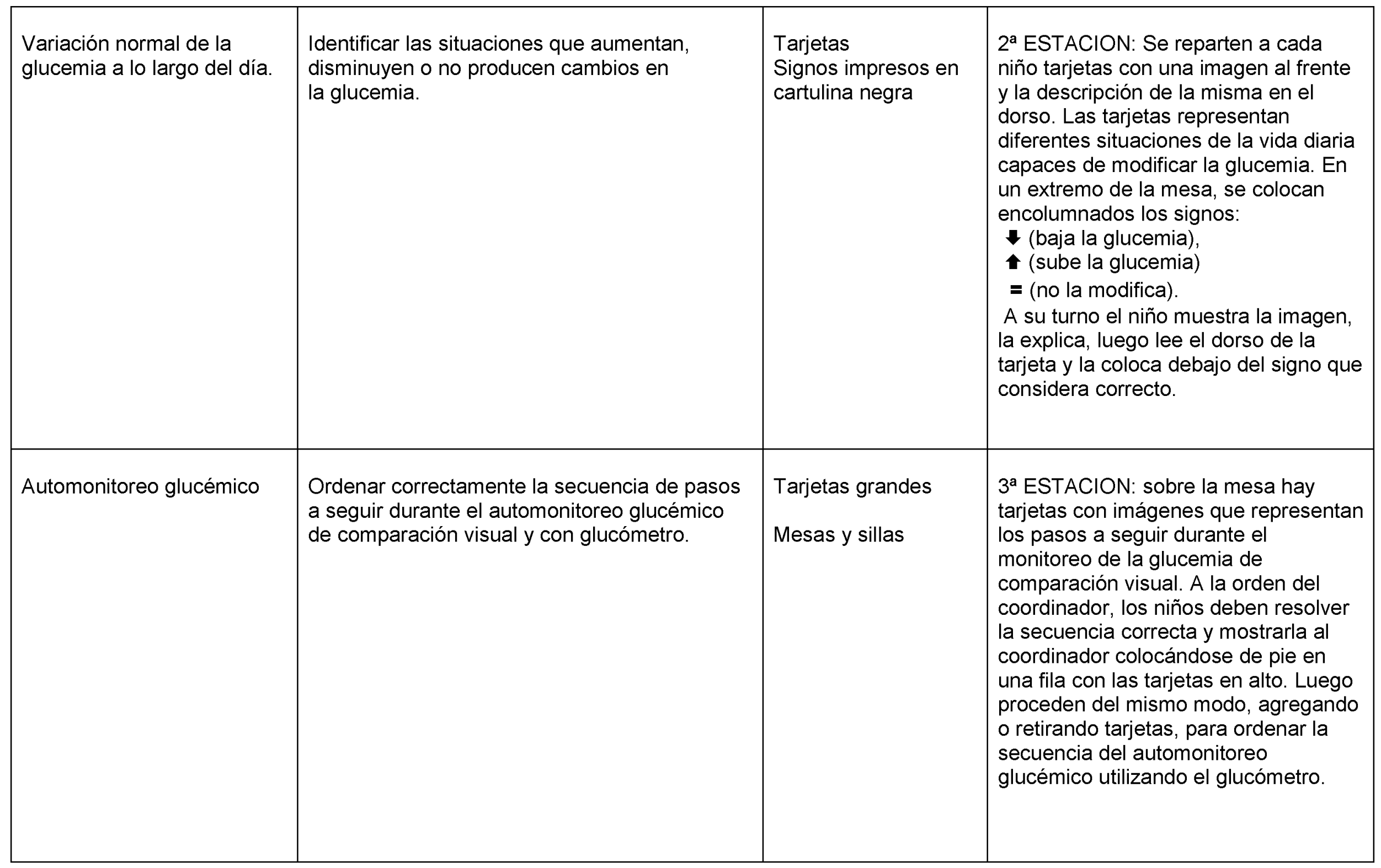




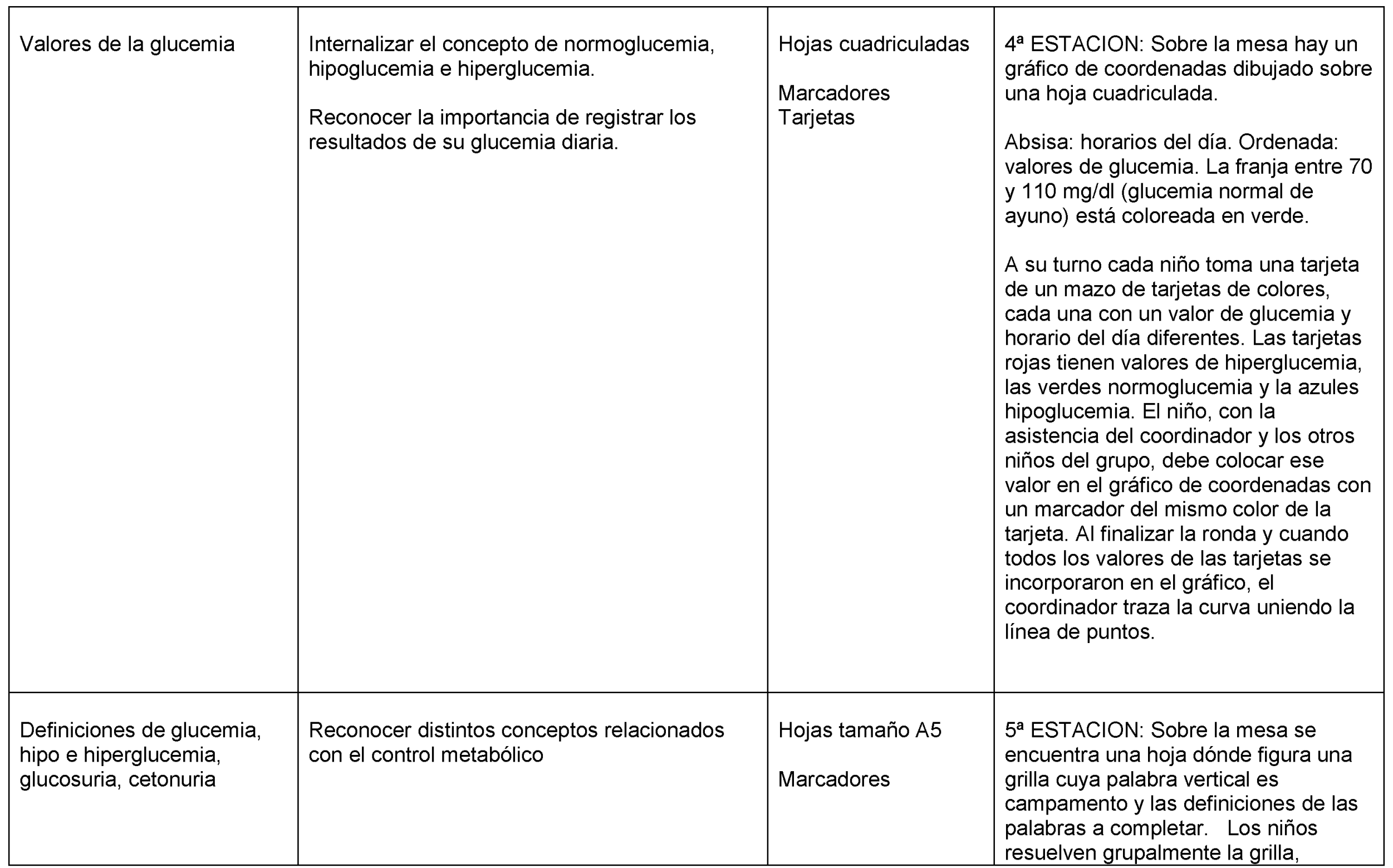




\begin{tabular}{|l|l|l|}
\hline & & $\begin{array}{l}\text { completando cada palabra ubicada } \\
\text { horizontalmente, luego de leer las } \\
\text { definiciones correspondientes. Estas } \\
\text { están referidas a los conceptos de } \\
\text { hipo e hiperglucemia, glucosuria, } \\
\text { cetonuria, etc. }\end{array}$ \\
\hline
\end{tabular}

Al finalizar cada estación el equipo de niños rotó a la siguiente.

El juego por estaciones fue precedido por una explicación de 15 minutos de duración, dónde se definieron los valores normales de glucemia de ayuno y postprandial, hipoglucemia e hiperglucemia. Se describieron las fuentes de energía que utiliza nuestro cuerpo en diferentes situaciones y el efecto del ejercicio a corto, mediano y largo plazo. Se explicó la importancia de mantener la glucemia dentro o lo más cercano posible a los valores normales y la importancia del automonitoreo frecuente de la glucemia y el control periódico de la hemoglobina glicosilada. Para ello se utilizaron láminas con la imagen de una balanza con los diferentes valores de euglucemia, hipoglucemia e hiperglucemia. 


\section{TEMA: INTEGRACION DE CONOCIMIENTOS SOBRE DIABETES}
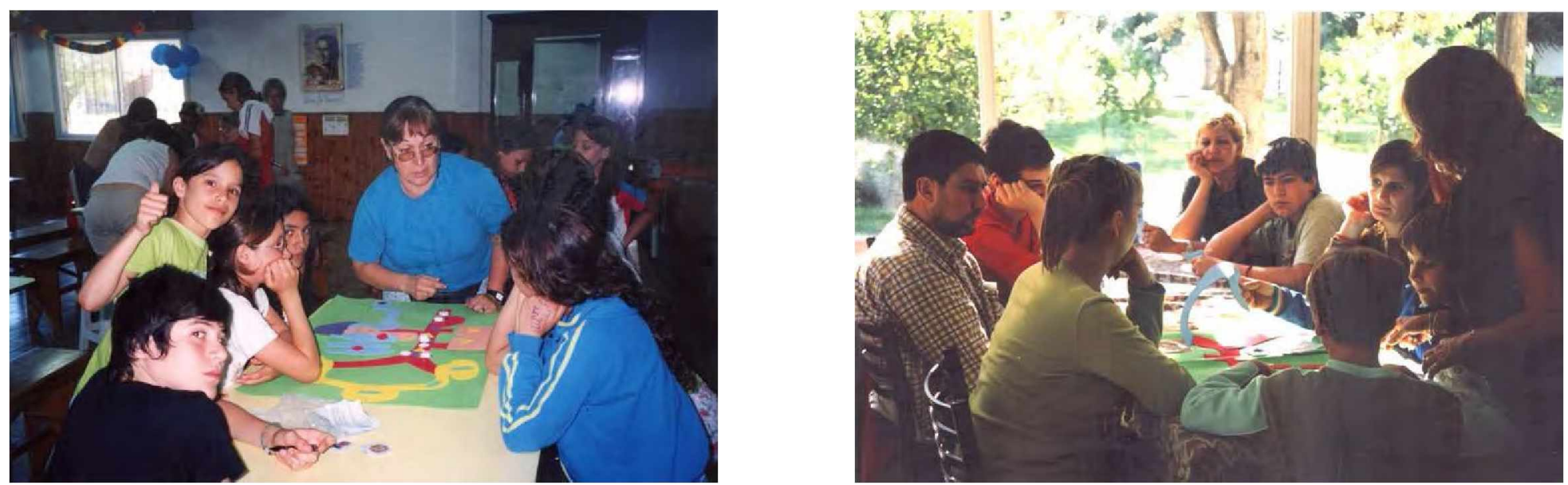

\begin{tabular}{|c|c|c|c|}
\hline CONTENIDOS & OBJETIVOS & MATERIALES & DESCRPCION \\
\hline $\begin{array}{l}\text { Metabolismo de la } \\
\text { glucosa, alimentación, } \\
\text { complicaciones crónicas } \\
\text { e importancia de los } \\
\text { controles periódicos. } \\
\text { Hábitos de vida } \\
\text { saludables: alimentación } \\
\text { sana, actividad física y } \\
\text { descanso. }\end{array}$ & $\begin{array}{l}\text { Comprender los mecanismos } \\
\text { involucrados en la regulación } \\
\text { del metabolismo de la glucosa y } \\
\text { de la glucemia. } \\
\text { Reconocer el porqué y el para } \\
\text { qué de los controles periódicos } \\
\text { clínicos y de laboratorio. } \\
\text { Relacionar la hiperglucemia } \\
\text { crónica con la aparición de } \\
\text { complicaciones tardías en } \\
\text { diferentes órganos. }\end{array}$ & $\begin{array}{l}\text { Tarjetas } \\
\text { Fotos de alimentos } \\
\text { Fichas (insulina, } \\
\text { glucosa, grasas, } \\
\text { proteínas y cetonas). } \\
\text { Contorno de la figura } \\
\text { humana y órganos } \\
\text { (estómago, ojos, } \\
\text { intestino, páncreas, } \\
\text { riñones, células, } \\
\text { corazón y sistema } \\
\text { circulatorio, en goma } \\
\text { eva de diferentes } \\
\text { colores. }\end{array}$ & $\begin{array}{l}1^{\mathrm{a}}, 2^{\mathrm{a}}, 3^{\mathrm{a}}, 4^{\mathrm{a}} \text { y } 5^{\mathrm{a}} \text { ESTACION: Desplegar sobre la mesa el } \\
\text { contorno de la figura humana (sin los miembros). Cada } \\
\text { niño lee en voz alta una tarjeta con una pregunta. Las } \\
\text { tarjetas se leen en orden previamente establecido pues las } \\
\text { preguntas van relacionando las distintas etapas } \\
\text { (masticación, digestión, absorción y distribución de las } \\
\text { distintas fuentes energéticas), desde el ingreso del } \\
\text { alimento hasta su metabolización y eliminación. Luego de } \\
\text { leer una tarjeta, los niños colocan las piezas como en un } \\
\text { juego de encastre (órganos) y las fichas (células, glucosa, } \\
\text { grasas, insulina, proteínas), según las indicaciones. } \\
\text { Según el contenido de la tarjeta, el coordinador, mueve las } \\
\text { piezas del juego generando situaciones problema, para } \\
\text { que el niño pueda integrar conocimientos y para explicar la } \\
\text { fisiopatología de la diabetes. }\end{array}$ \\
\hline
\end{tabular}

NOTA: El juego fue recreado y adaptado del "BODYLINK" inventado por el Dr.Martyn Sulway, Royal North Shore Hospital Sydney.

(Boehringer Mannheim, Australia) 


\section{TEMA: SELECCION Y CONTEO DE CARBOHIDRATOS}
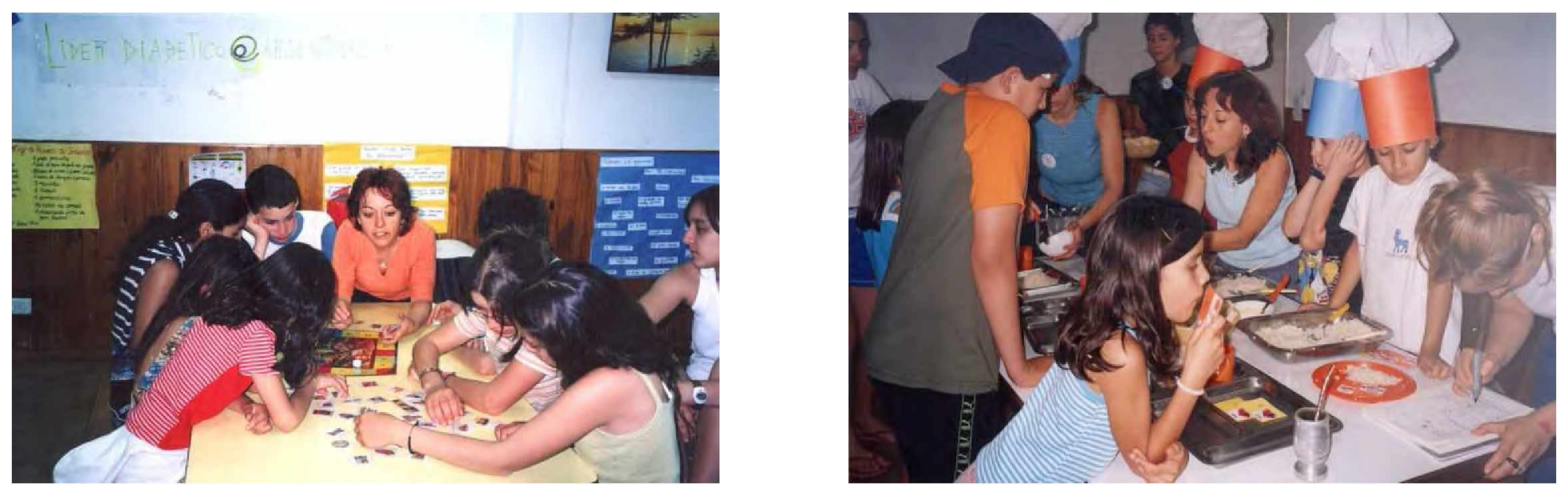

\begin{tabular}{|l|l|l|l|}
\hline \multicolumn{1}{|c|}{ CONTENIDOS } & \multicolumn{1}{|c|}{ OBJETIVOS } & \multicolumn{1}{|c|}{ MATERIALES } & \multicolumn{1}{c|}{ DESCRPCION } \\
\hline $\begin{array}{l}\text { Identificación del } \\
\text { contenido de } \\
\text { carbohidratos } \\
\text { (cantidad y tipo) de } \\
\text { los alimentos. }\end{array}$ & $\begin{array}{l}\text { Reconocer los componentes } \\
\text { nutritivos en diferentes } \\
\text { alimentos. }\end{array}$ & $\begin{array}{l}\text { Tarjetas } \\
\text { Identificar los alimentos que } \\
\text { modifican la glucemia. }\end{array}$ & $\begin{array}{l}\text { Cartas con fotografias de } \\
\text { alimentos } \\
\text { Seleccionar los alimentos más } \\
\text { apropiados para consumir en } \\
\text { una situación particular. }\end{array}$ \\
$\begin{array}{l}\text { Alimentos plásticos } \\
\text { Cuantificar los carbohidratos } \\
\text { en distintas porciones de } \\
\text { alimentos. }\end{array}$ & $\begin{array}{l}\text { Mesas y sillas } \\
\text { Láminas }\end{array}$ & $\begin{array}{l}\text { alimentos. En un extremo de la mesa se encuentran } \\
\text { encolumnadas las palabras Hidratos de carbono, Proteínas y } \\
\text { Grasas. Por turno cada niño da vuelta la carta y coloca la } \\
\text { imagen del alimento en la columna que considera correcta. } \\
\text { 2a ESTACION: Sobre la mesa se coloca una lámina con la } \\
\text { imagen de una pirámide de base color verde (no modifica la } \\
\text { glucemia), amarilla en el centro (eleva la glucemia } \\
\text { lentamente) y roja en la parte superior (eleva la glucemia } \\
\text { rápidamente). Cada niño por turno extrae al azar de una } \\
\text { bolsa, un alimento plástico que debe colocar en alguna zona } \\
\text { de color de la pirámide. }\end{array}$ \\
\hline
\end{tabular}




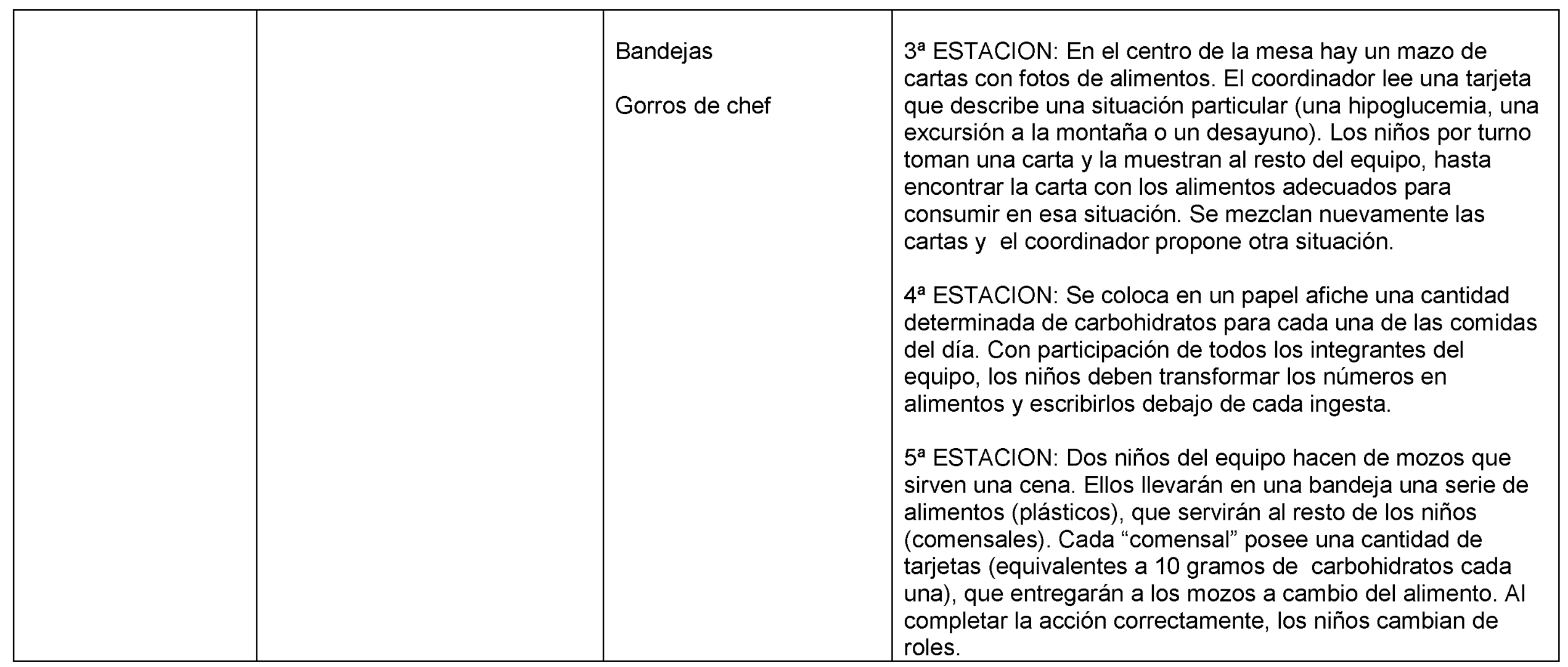




\section{TEMA: IDENTIFICACION DE NUTRIENTES}

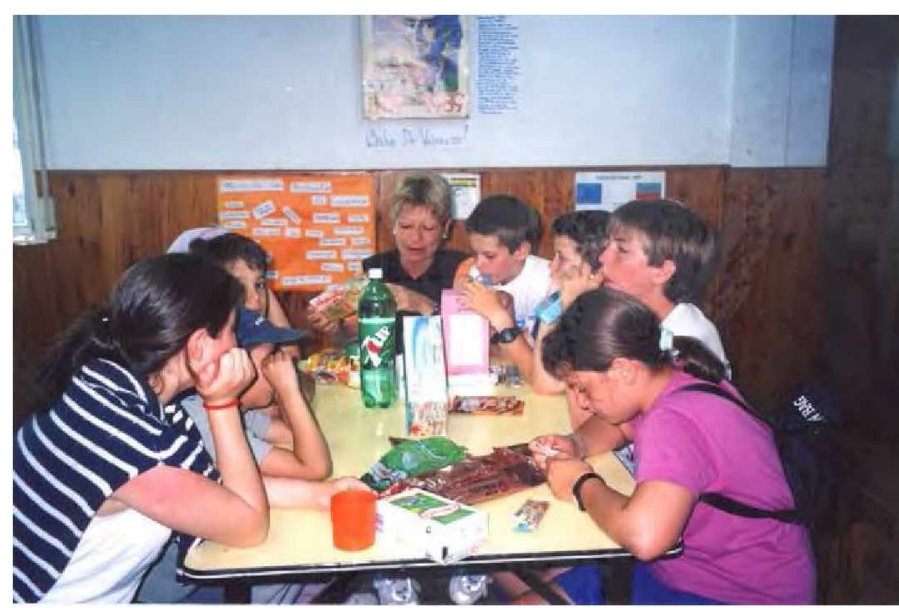

\begin{tabular}{|c|c|c|c|}
\hline CONTENIDOS & OBJETIVOS & MATERIALES & DESCRPCION \\
\hline $\begin{array}{l}\text { Reconocimiento de } \\
\text { macronutrientes en } \\
\text { diferentes alimentos }\end{array}$ & $\begin{array}{l}\text { Reconocer los sinónimos } \\
\text { utilizados para diferentes } \\
\text { sustancias alimenticias } \\
\text { (ej:carbohidratos, proteínas, } \\
\text { lípidos, fibra alimentaria). } \\
\text { Identificar el tipo de } \\
\text { carbohidrato de cada } \\
\text { alimento (complejos y } \\
\text { simples). } \\
\text { Detectar la presencia de } \\
\text { TACC (trigo, avena, cebada } \\
\text { y centeno) en los alimentos. }\end{array}$ & $\begin{array}{l}\text { Tarjetas con preguntas y } \\
\text { consignas } \\
\text { Envases de alimentos } \\
\text { comunes y dietéticos } \\
\text { Alimentos plásticos } \\
\text { Mesas y sillas } \\
\text { Láminas }\end{array}$ & $\begin{array}{l}1^{\mathrm{a}}, 2^{\mathrm{a}}, 3^{\mathrm{a}}, 4^{\mathrm{a}} \text { y } 5^{\mathrm{a}} \text { ESTACION: } \\
\text { Los niños se agrupan alrededor de } 5 \text { mesas. En cada una se } \\
\text { hallan distribuidos diferentes tipos de alimentos y envases. } \\
\text { Ej: mermeladas comunes y diet, galletas de arroz, galletas de } \\
\text { trigo, harinas de diferentes tipos, polvos para postres y } \\
\text { gelatinas, caldos y sopas, edulcorantes líquidos y en polvo, } \\
\text { gaseosas y jugos comunes y diet, etc. } \\
\text { Los niños exploran los alimentos libremente y luego cada } \\
\text { niño lee en voz alta una tarjeta con una pregunta, que el } \\
\text { equipo deberá responder. } \\
\text { Preguntas (algunos ejemplos): }\end{array}$ \\
\hline
\end{tabular}




\begin{tabular}{|l|l|l|l|}
\hline & $\begin{array}{l}\text { Distinguir la diferencia entre } \\
\text { alimentos dietéticos y Light. }\end{array}$ & $\begin{array}{l}\text { ¿Qué alimentos contienen fibra? } \\
\text { ¿Cuál es la diferencia entre los alimentos comunes y los } \\
\text { dietéticos? } \\
\text { ¿Cuál es la diferencia entre los alimentos dietéticos y los } \\
\text { alimentos light? } \\
\text { ¿Qué es un edulcorante? }\end{array}$ \\
\hline
\end{tabular}

Como parte de la educación alimentaria, se utilizó en las cuatro comidas del CND y del EF, un sistema de tarjetas de diferente color e igual valor: $10 \mathrm{~g}$ de carbohidratos. Cada color representó a un grupo de alimentos proveedor de carbohidratos: leche o yogur (tarjeta celeste), fruta (tarjeta amarilla) y alimentos feculentos, legumbres y cereales (tarjeta naranja). Antes de cada comida y según el valor de glucemia, la dosis de insulina a recibir, la actividad física desarrollada y la programada posteriormente, el momento del día, cada niño recibió de la nutricionista, la cantidad de tarjetas correspondientes a los gramos de carbohidratos que necesitaba consumir en la siguiente ingesta.

Cada día durante el CND, un equipo diferente de niños (y de adultos durante el EF), supervisados por miembros del equipo, sirvieron los alimentos al resto de los niños de acuerdo a la cantidad de tarjetas que les entregaron. Para ello, el área de servicio de comidas se dividió en 3 sectores: a) sector de alimentos de consumo libre (hortalizas), b) sector de alimentos proteicos (carnes y quesos) y c) sector de alimentos proveedores de carbohidratos (alimentos feculentos, cereales, legumbres y frutas). En el sector c se exhibió una porción equivalente a $10 \mathrm{~g}$ de carbohidratos medidos en cucharas, tazas y unidades, para que los niños visualizaran el volumen correspondiente a la porción mencionada. En dicho sector se ofrecieron distintos tipos de alimentos para que los niños pudieran optar por uno o más de ellos. 


\section{Fuentes y Tipo de Información}

Los datos evaluados se obtuvieron de las siguientes fuentes de información:

a- Formulario de inscripción al CND y EF (Anexos 4 y 5): en ellos se registraron: datos personales, nivel educativo, cobertura de salud, antigüedad de la diabetes, talla y peso, tipo de control y de tratamiento, hábitos y frecuencia de actividad física extraescolar, plan de alimentación; habilidades para el automonitoreo glucémico y glucosúrico, preparación y autoinyección de insulina, rotación de los sitios de inyección. El formulario incluyó una planilla de registro de la insulinoterapia, valores y frecuencia del monitoreo glucémico y glucosúrico de la semana previa al CND y EF, y HbA1c de 10 días antes del CND y del EF, para ser entregada el día del inicio de ambos eventos.

Estos formularios de inscripción al CND se enviaron cinco meses antes del campamento, a distintos centros de salud, médicos pediatras y endocrinólogos, asociaciones de personas con diabetes y al domicilio particular de aquellos niños que habian participado de un campamento.

Los formularios de inscripción al EF se enviaron con tres meses de anticipación al encuentro sólo a aquellas familias cuyos niños habían participado del CND. Se solicitó que el formulario lo completara un familiar adulto o tutor del niño y que fuera entregado 1-2 meses antes de los eventos correspondientes. 
b- Formulario de seguimiento individual (Anexo 6): en él se registraron diariamente los esquemas de insulinoterapia, los valores de control metabólico (glucemia, glucosuria y cetonuria); la intensidad, frecuencia y tipo de actividad física desarrollada y la cantidad y distribución de carbohidratos a lo largo del día. El formulario incluyó un registro de evaluación de habilidades (Anexo 7), para el automonitoreo glucemico/glucosúrico, la preparación y autoinyección y la rotación de los sitios de inyección de insulina. El registro de habilidades se completó al ingreso y al egreso de los niños al CND y al ingreso de los niños al EF. Todos estos datos fueron registrados por miembros del equipo interdisciplinario durante el CND y el EF.

Al finalizar el CND y el EF se remitió una copia del formulario a la familia y al médico de cabecera de cada niño participante.

c- Planilla de registro (Anexo 8): de insulinoterapia, valores y frecuencia del monitoreo glucémico y glucosúrico, de la semana posterior al CND y $E F$.

d- Cuestionario de conocimientos (Anexo 9): 20 preguntas de selección múltiple referidas a: definición de diabetes, valores de normoglucemia, plan de alimentación, actividad física, insulinoterapia, complicaciones agudas, síntomas de hipoglucemia e hiperglucemia y automonitoreo glucémico. Se empleó el mismo cuestionario para los niños y el familiar adulto. 
Para evaluar los cambios inducidos por el CND y el EF, se compararon los resultados correspondientes a los distintos parámetros registrados durante el período de estudio, de la siguiente forma:

- Habilidades para la realización correcta de los distintos pasos del automonitoreo glucémico/glucosúrico, la preparación (secuencia de la carga de insulina de diferente tiempo de acción y análogos) y la autoinyección de insulina. La rotación de los sitios de inyección, y la selección de alimentos (identificación de la cantidad y el tipo de carbohidratos que contienen los alimentos) se evaluaron independientemente de las 4 habilidades anteriores. Todas las variables mencionadas se verificaron el primero y último día del CND y 4 meses después del mismo.

Para la evaluación estadística de las variables automonitoreo glucémico y glucosúrico, preparación y autoinyección de insulina y selección de alimentos, se realizó la siguiente clasificación: Lo puede hacer solo (LPHS), Necesita ayuda (NA), No lo hace (NLH).

Para la variable rotación de los sitios de inyección se consideraron 1,2 y $>2$ zonas de inyección, independientemente de cual fuera la zona utilizada. Cada zona representó dos lugares del cuerpo para la aplicación de insulina. 
- Conocimientos (n y porcentaje de respuestas correctas) sobre la enfermedad, su control, prevención y tratamiento; verificados en los niños el primero, el último día del CND y luego de 4 meses de su finalización; en los familiares adultos en ambos días del EF.

- Indicadores de control metabólico:

- Hba1c (\%) antes, 4 y 7 meses después del CND. Para la cuantificación de la HbA1c se utilizó el método del DCA 2000 (Bayer) cuyo valor normal de referencia es hasta $6,0 \%$.

- Glucemia (mg/dl) de automonitoreo (frecuencia y valores promedio diarios), realizadas durante los 6 días del CND en cuatro horarios (antes del desayuno, almuerzo, merienda y cena), y las realizadas en forma escalonada en diferentes horarios durante 6 días previos y posteriores al CND. Para su determinación se utilizaron glucómetros y tiras reactivas Onetouch-ultra (Johnson \& Johnson Medical S.A.).

- Episodios de hipoglucemia leve a moderada (n), durante el CND y los registrados en la semana anterior y posterior al CND.

- Indicadores de control clínico y tratamiento:

- Evaluación antropométrica: Peso $(\mathrm{kg})$ y Talla $(\mathrm{cm})$ se realizó con balanza con tallímetro incorporado marca CAM, al ingreso de los niños al CND y 4 meses después. La talla se expresó 
en escore $Z$ de talla de acuerdo a los estándares argentinos: valor de referencia normal $\pm 1.8(67)$.

- Indice de masa corporal (IMC) $\mathrm{kg} / \mathrm{m} 2$ se expresó en escore Z de IMC: valor de referencia normal $\pm 1.8(68)$

Signos locales de lipodistrofia en los sitios de aplicación de insulina, durante y 4 meses después del CND.

- Tipo y cantidad de insulina (unidades/dosis/día) y número de inyecciones/día de la semana anterior, durante el CND, y en la semana posterior al CND. Para la evaluación estadística, el tipo de insulina se clasificó según su tiempo de acción en: insulina de acción rápida (rápida y ultrarrápida) e insulina de acción prolongada (NPH y Glargina).

Para la evaluación estadística de las variables conocimientos e indicadores de control clínico, metabólico y de tratamiento se dividió a la población de estudio en dos grupos: con cobertura (niños que tenían cobertura de salud brindada por la seguridad social o el sistema de medicina prepaga) y niños que recibían los beneficios del sector público (Programa de Diabetes de la Provincia de Buenos Aires - PRODIABA - Ministerio de Salud de la Provincia de Buenos Aires). Con fines descriptivos se definió a este segundo grupo como sin cobertura. 


\section{Análisis Estadistico}

Los datos se registraron en una planilla de cálculo Excel 2000, los registros se incorporaron en una base de datos ad hoc y se analizaron empleando el software Statgrafic. En el análisis estadístico se consideró un nivel de confianza del 95\% y para determinar la significación estadística de las diferencias registradas se utilizó el el análisis de la varianza (ANOVA) y el método de Student (t) para variables continuas (comparación de promedios para datos no apareados) y el test de Tukey cuando se compararon más de dos grupos entre sí. Para comparar porcentajes se empleó el test de $x^{2}$. 
RESULTADOS d 
Las características de los niños que participaron del Campamento para niños con diabetes (CND) se resumen en la Tabla 1. Los datos se obtuvieron del formulario de inscripción al CND y de la planilla de registro del tratamiento de la semana anterior al mismo.

TABLA 1. Características de los niños que participaron del CND

\begin{tabular}{|c|c|c|c|}
\hline & CND & Con Cobertura & Sin Cobertura \\
\hline $\operatorname{Niños~(n)~}$ & 37 & 22 & 15 \\
\hline Edad (años) & $10.8 \pm 1.6$ & $10.8 \pm 1.6$ & $10.8 \pm 1.7$ \\
\hline Sexo (\% mujeres) & $51.4 \%$ & $50 \%$ & $53.3 \%$ \\
\hline Escolaridad (año EGB) & $5.3 \pm 1.6$ & $5.4 \pm 1.6$ & $5.3 \pm 1.7$ \\
\hline Antigüedad de Diabetes (años) & $3.4 \pm 2.8$ & $3.1 \pm 2.8$ & $3.9 \pm 2.9$ \\
\hline Actividad Física Extraescolar (\%) & $70.3 \%$ & $77.3 \%$ & $60 \%$ \\
\hline $\begin{array}{l}\text { Conocimientos (niños) } \\
\text { (número de respuestas correctas) }\end{array}$ & $16 \pm 3$ & $16 \pm 3$ & $16 \pm 3$ \\
\hline $\begin{array}{l}\text { Conocimientos (familiares) } \\
\text { (número de respuestas correctas) }^{*}\end{array}$ & $18 \pm 2.1$ & $19 \pm 2$ & $17 \pm 2$ \\
\hline \multicolumn{4}{|l|}{ Nivel de estudios de los padres (n) } \\
\hline Primario incompleto & 1 & - & 1 \\
\hline Primario & 8 & 1 & 7 \\
\hline Secundario & 19 & 12 & 7 \\
\hline Terciario o universitario & 9 & 9 & - \\
\hline Escore $\mathrm{Z}$ de IMC & $+0.63 \pm 1.18$ & $+0.50 \pm 1.12$ & $+0.82 \pm 1.29$ \\
\hline Escore $\mathrm{Z}$ de talla & $+0.27 \pm 1.05$ & $+0.35 \pm 1.01$ & $+0.15 \pm 1.14$ \\
\hline $\mathrm{HbA1c}(\%)$ & $10.3 \pm 2.3$ & $10.1 \pm 2.4$ & $10.7 \pm 2.3$ \\
\hline \multicolumn{4}{|l|}{ Tratamiento } \\
\hline Dosis total insulina (U/día) & $44 \pm 27$ & $47 \pm 31$ & $40 \pm 19$ \\
\hline Dosis insulina prolongada (U/día) & $39 \pm 23$ & $40 \pm 27$ & $37 \pm 18$ \\
\hline Dosis insulina rápida (U/día) & $6 \pm 8$ & $8 \pm 10$ & $3 \pm 2$ \\
\hline Número de inyecciones/día ** & $2 \pm 1$ & $3 \pm 1$ & $2 \pm 1$ \\
\hline $\begin{array}{l}\text { Número de autodeterminaciones } \\
\text { de glucemia/día*** }\end{array}$ & $3 \pm 1$ & $3 \pm 1$ & $2 \pm 1$ \\
\hline
\end{tabular}

Escore $Z$ de IMC (índice de masa corporal); Escore $Z$ de talla según las Tablas Argentinas de Crecimiento de Población (valor normal: \pm 1.8 )

* $p$ : 0,05 niños con vs. sin cobertura; ${ }^{* *}$ p: 0,02 niños con vs. sin cobertura *** p: 0,001 niños con vs. sin cobertura.

Los resultados se expresan como media \pm DE (desvío estándar), porcentaje y frecuencia.

El $59.5 \%$ de los niños participantes tenía cobertura de salud (con cobertura), mientras que el $40.5 \%$ restante pertenecía al grupo definido como 
sin cobertura. Mientras los niños con y $\sin$ cobertura recibían la provisión completa de la insulina prescripta para su tratamiento, no ocurrió lo mismo con la provisión de tiras reactivas para automonitoreo glucémico: los niños con cobertura recibieron un promedio mensual de $110 \pm 27$ tiras para lectura con glucómetro pero los niños sin cobertura recibieron en ese período alrededor de 33 tiras de comparación visual. De estos últimos, sólo en 4 casos sus familiares informaron poder comprar 25 tiras de iguales características en algunos de los meses del año, por lo que estos niños disponían de un promedio de $44 \pm 23$ tiras/mes. En consecuencia la cantidad de tiras y el número de determinaciones de glucemia diarias en este grupo fueron significativamente menores que la de los niños con cobertura (p 0.001). En efecto, mientras el $64 \%$ de los niños con cobertura realizaba 4 o más determinaciones diarias, sólo 1 niño sin cobertura realizaba este número de determinaciones, y el $73 \%$ de los niños sin cobertura realizaba 1-2 determinaciones de glucemia diarias.

De los 37 niños que asistieron al CND, 11 (30\%) realizaban tratamiento intensificado con 3 o más inyecciones diarias de insulina, de los cuales $9(82 \%)$ pertenecian al grupo con cobertura; los 26 niños restantes (70\%), utilizaban 1 ó 2 inyecciones diarias (tratamiento convencional), de los cuales el 50\% (13) pertenecían al grupo sin cobertura.

Al ingreso, tanto la $\mathrm{HbA} 1 \mathrm{c}$ como el escore $\mathrm{Z}$ de IMC, fueron similares en los niños con y sin cobertura. En ambos grupos el escore $Z$ de talla se encontraba dentro de límites normales para la población argentina $( \pm 1.8)$. Estos datos indican que en el período previo al CND ambos grupos tenían un grado de control clínico y metabólico similar. 


\section{Características de los niños participantes y sus familiares}

De los 37 niños que participaron del CND, el 51\% (19 niños) asistieron al Encuentro familiar (EF) junto a 19 familiares adultos (16 madres y 3 padres). El tiempo transcurrido entre las dos intervenciones educativas (CND Y EF) fue de 4 meses.

El 53\% de los participantes tanto del CND como del EF fueron niñas. El promedio de edad de los niños participantes también fue similar para el CND y el EF $(10.8 \pm 1.6$ y $10.9 \pm 1.7$ años respectivamente $)$

El 59.5\% (22 niños) de los niños participantes del CND lo hicieron por primera vez, mientras que el $40.5 \%$ restante (15 niños) participaron por segunda vez consecutiva. Los 19 niños y 19 adultos familiares asistieron por primera vez a un EF.

La antigüedad de la diabetes en los niños participantes oscilaba entre $<1$ año (5 niños del CND y 3 del EF) y 10 años.

La totalidad de los niños del CND y del EF cursaban el nivel de EGB (Educación General Básica): CND: 19\% en el primer ciclo, 51\% en el segundo y $30 \%$ en el tercer ciclo; EF: $47 \%$ en el segundo ciclo y $53 \%$ en el tercero. El nivel educativo de los familiares de los niños participantes fue similar en ambos eventos.

De los 19 niños que participaron del EF, 17 (89.5\%) practicaba actividad física extraescolar vs. 26 (70\%) de los que asistieron al CND.

Participaron del EF, 11 (58\%) niños con cobertura y $8(42 \%) \sin$ cobertura. En la evaluación 4 meses post campamento, de los 19 niños que asistieron al EF el 32\% empleaba tratamiento intensificado (3 ó más 
inyecciones diarias de insulina), mientras que el $68 \%$ utilizaba 1 ó 2 inyecciones diarias (tratamiento convencional); el promedio general fue de $2 \pm$ 1 inyecciones diarias. El número de monitoreos glucémicos y la dosis de insulina diaria, fueron similares en los niños que participaron del CND y del EF.

Comparación entre las semanas anterior, durante y posterior al campamento:

La comparación de las variables clínico-metabólicas entre las semana anterior y posterior al CND y durante el CND, se describen en la Tabla 2.

TABLA 2. Tratamiento: Comparación de sus características antes, durante y después del CND

\begin{tabular}{|l|c|c|c|c|}
\hline & $\begin{array}{c}\text { Semana } \\
\text { anterior CND } \\
(\mathbf{n}: 37)\end{array}$ & $\begin{array}{c}\text { Semana CND } \\
\text { (n:37) }\end{array}$ & $\begin{array}{c}\text { Semana } \\
\text { posterior CND } \\
\text { (n:36) }\end{array}$ & Valor P \\
\hline $\begin{array}{l}\text { Dosis total insulina } \\
\text { (U/dia) }\end{array}$ & $44 \pm 27$ & $41 \pm 21$ & $41 \pm 24$ & NS \\
\hline $\begin{array}{l}\text { Dosis insulina } \\
\text { prolongada } \\
\text { (U/dia) }\end{array}$ & $39 \pm 23$ & $36 \pm 22$ & $35 \pm 21$ & NS \\
\hline $\begin{array}{l}\text { Dosis insulina rápida } \\
\text { (U/día) }\end{array}$ & $6 \pm 8$ & $5 \pm 4$ & $7 \pm 7$ & NS \\
\hline $\begin{array}{l}\text { Número de } \\
\text { inyecciones/dia }\end{array}$ & $2 \pm 1$ & $2 \pm 1$ & $2 \pm 1$ & NS \\
\hline $\begin{array}{l}\text { Número de } \\
\text { autodeterminaciones } \\
\text { Glucemia/día }\end{array}$ & $3 \pm 1$ & $4 \pm 0.2$ & $3 \pm 1$ & $<0.001$ \\
\hline $\begin{array}{l}\text { Frecuencia de } \\
\text { hipoglucemia } \\
\text { (día/niño) }\end{array}$ & $0.1 \pm 0.2$ & $0.7 \pm 0.6$ & $0.1 \pm 0.2$ & $<0.001$ \\
\hline Glucemia (mg/dl) & $178 \pm 59.5$ & $137.4 \pm 33.7$ & $177.5 \pm 44.1$ & $<0.001$ \\
\hline
\end{tabular}

NS: no significativo ( $\mathrm{p}:>0.05)$

Los resultados se expresan como media $\pm \mathrm{DE}$ (desvío estándar) 
Allí puede verse que la dosis media total de insulina diaria, la de acción prolongada y el número diario de inyecciones fue similar en la semana previa al CND, durante el CND y en la semana posterior al mismo.

Aunque durante la semana del CND y en la semana posterior se registró una disminución de 2 unidades en la dosis promedio de insulina de acción rápida en comparación con la dosis de la semana anterior, las diferencias no fueron estadísticamente significativas.

El número de determinaciones diarias de glucemia fue 1.16 veces mayor durante el CND comparado con las de la semana anterior $(p<0.001)$ y 0.58 veces mayor de las de la semana posterior $(p<0.005)$. Teniendo en cuenta las determinaciones de glucemia realizadas fuera de las programadas diariamente (las realizadas ante síntomas de hipoglucemia), el promedio durante el CND fue de $5 \pm 1$ determinaciones por niño/día.

El valor promedio de glucemia durante el CND fue $41 \mathrm{mg} / \mathrm{dl}$ y 40 $\mathrm{mg} / \mathrm{dl}$ menor que el de la semana anterior y posterior respectivamente (en ambos casos $p<0.001$ ).

El número de hipoglucemias (leves a moderadas) diarias por niño durante el CND fue mayor al de las registradas durante las semanas anterior y posterior al campamento $(p<0.001)$. Durante el CND, el $41 \%$ de estos episodios de hipoglucemia ocurrió en momentos del día en que la duración e intensidad de la actividad física fueron mayores. Las hipoglucemias nocturnas durante el CND representaron el $11 \%$ del total de hipoglucemias registradas.

No registramos episodios de hipoglucemia severa ni de cetoacidosis durante el CND ni en la semana anterior y posterior al mismo. Tampoco hubo episodios de este tipo durante el EF. 


\section{Conocimientos y habilidades:}

Empleando un cuestionario descripto previamente evaluamos los conocimientos de los niños respecto al control y tratamiento de la diabetes en tres momentos: primer día del CND (pre campamento), último día del CND (campamento) y 4 meses después del CND (post campamento). Este último correspondió al primer día del EF. En los mismos momentos también evaluamos 4 habilidades: automonitoreo glucémico y glucosúrico, preparación y auto inyección de insulina (Tabla 3).

TABLA 3. Conocimientos y habilidades pre y post campamento

\begin{tabular}{|c|c|c|}
\hline & $\begin{array}{c}\text { Número de } \\
\text { Respuestas } \\
\text { correctas } \\
\text { (n:37) }\end{array}$ & $\begin{array}{c}\text { Número de } \\
\text { Habilidades } \\
\text { (n:37) }\end{array}$ \\
\hline Pre campamento & $16 \pm 3$ & $3 \pm 1$ \\
\hline Campamento & $18 \pm 2$ & $4 \pm 1$ \\
\hline Post campamento & $18 \pm 2$ & $4 \pm 1$ \\
\hline Valor de P & $<0.001$ & 0.004 \\
\hline
\end{tabular}

Los resultados se expresan como media $\pm \mathrm{DE}$

Número de respuestas correctas: $p: 0,01$ primero vs. último día de campamento y p: 0,04 primer día vs. 4 meses post campamento. Número de habilidades: p: 0,01 primero vs. último día de campamento y p: 0,009 primer día vs. 4 meses post campamento.

El porcentaje de respuestas correctas aumentó significativamente en el último día del CND (91 $\pm 11 \% ; p<0.001)$ y a los 4 meses $(90 \pm 10 \% ; p$ <0.004) comparado con el del primer día $(80 \pm 14 \%)$. No registramos 
diferencias significativas entre el último día del CND y la evaluación post campamento.

Algo similar ocurrió con las habilidades antes mencionadas: su número fue significativamente mayor el último día del CND y 4 meses después comparados con el registrado el primer día del CND.

No registramos diferencias significativas entre los conocimientos y habilidades de niños (registrados el primer día del CND) que concurrieron por primera y por segunda vez al CND.

Tampoco encontramos diferencias significativas en el número de respuestas correctas (evaluación del primer día) de los 19 niños y 19 familiares adultos que participaron del EF y las de los adultos (n:18) y niños (n:18) que no participaron. Sin embargo en los adultos que asistieron al EF observamos un incremento no significativo del porcentaje de respuestas correctas: $90 \pm$ $13 \%$ al inicio vs. $96 \pm 8 \%$ al término del mismo.

El número de respuestas correctas registradas el primer día del CND fue significativamente mayor en los niños de 10-13 años $(17 \pm 2)$ que el correspondiente a los de $7-9$ años $(13 \pm 3 ; \quad p<0.001)$. Esta diferencia a favor de los niños mayores (10-11 y 12-13 años) también se observó en el incremento de respuestas correctas registrado el último día del CND: $17 \pm 3$ vs. $19 \pm 2$ y $18 \pm 2$ respectivamente, $p<0.02$. En cambio no hubo tal diferencia en la evaluación realizada 4 meses post campamento.

El número de habilidades no mostró diferencias significativas asociadas a la edad de los niños o la duración de su enfermedad.

Por su especificidad e impacto sobre el control metabólico, la selección de alimentos y rotación de los sitios de inyección se evaluaron 
separadamente del área de los conocimientos y el de las habilidades. En el período previo al CND el $76 \%$ de los niños utilizaba más de 2 zonas para inyectarse insulina, porcentaje que a su término aumentó al $100 \%(p<0.005)$ y volvió a disminuir al 89\% 4 meses después.

El primer día de campamento, sólo el $38 \%$ de los niños identificaba la cantidad y tipo de carbohidratos de los alimentos (selección de alimentos), mientras que el último día ese porcentaje aumentó al 73\% ( $p<0.01)$ y al $68 \%(p$ $<0.03$ ) en la evaluación post campamento.

Tanto en el pre como en la evaluación post campamento, no registramos diferencias significativas entre los conocimientos y habilidades de niños con y sin cobertura. Sin embargo, el número de respuestas correctas registradas a los 4 meses post campamento fue significativamente mayor en los 22 familiares de los niños con cobertura que el correspondiente a los 15 familiares del grupo sin cobertura ( $p$ 0.05). No registramos esta diferencia entre estos dos grupos de adultos familiares que participaron del EF, durante la segunda evaluación de conocimientos (último día del EF).

Indicadores clínico-metabólicos antes y después del CND y del EF:

Al inicio del CND, 4 niños presentaban sobrepeso y 3 obesidad (según el escore $Z$ de IMC: $+2.15 \pm 0.29$ y $+3.10 \pm 0.95$ respectivamente). El escore $Z$ de talla se encontraba, dentro de valores normales de acuerdo a los estándares argentinos (+0.27 \pm 1.05$)$.

Aunque en la evaluación post campamento (4 meses) no se registraron cambios significativos ni en el escore $Z$ de talla ni en el escore $Z$ de IMC, 2 de los niños con sobrepeso y 1 con obesidad normalizaron su IMC. 
Sólo en 1 niño registramos signos de lipodistrofia hipertrófica en el brazo izquierdo al ingreso al campamento. No hicimos evaluación post campamento de este niño porque no asistió al EF.

TABLA 4. Valores de HbA1c (\%)

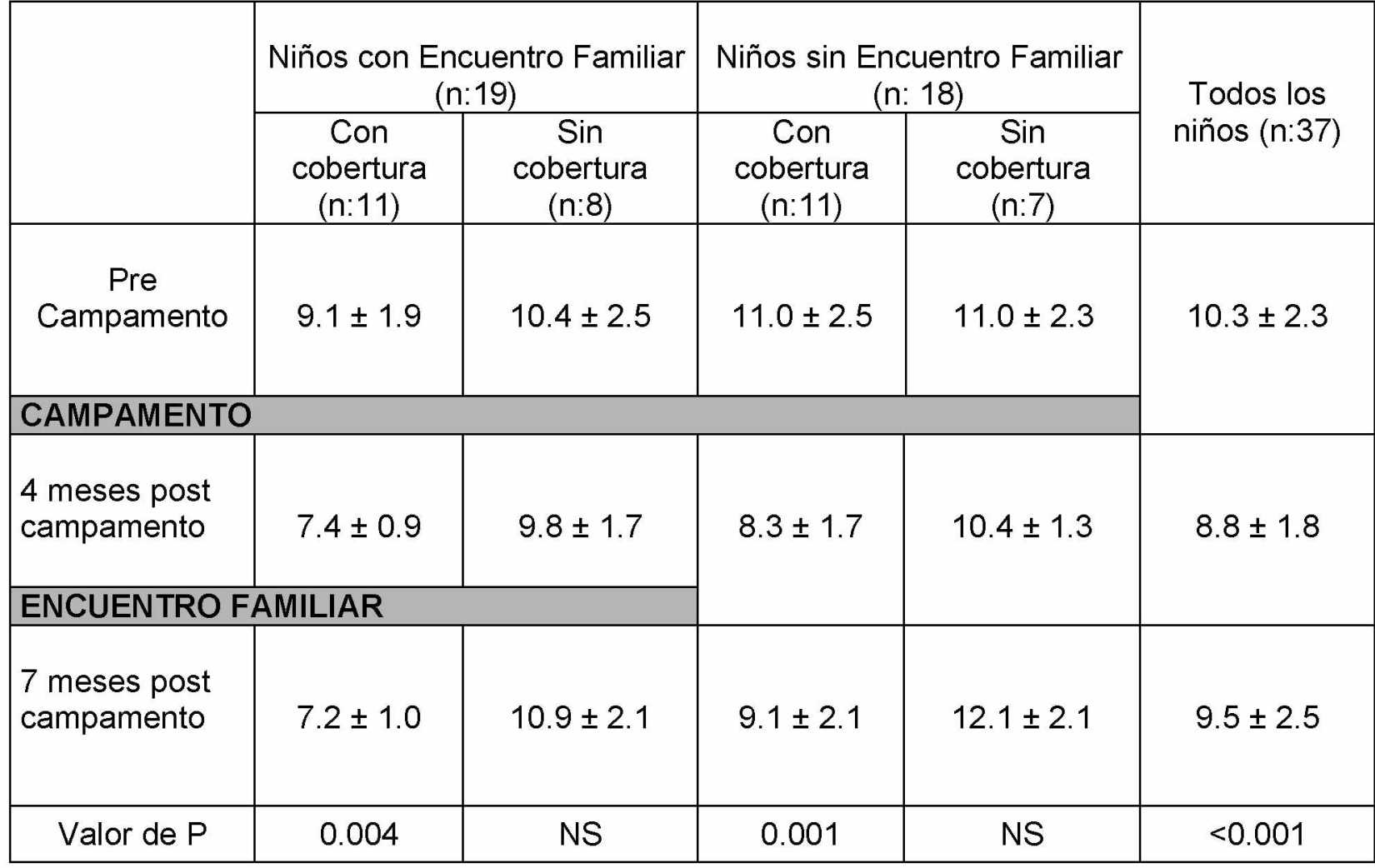

NS: no significativo, $\mathrm{p}:>0.05$

Los resultados se expresan como media $\pm \mathrm{DE}$ (desvío estándar)

La tabla 4 compara los valores de $\mathrm{HbA} 1 \mathrm{c}$ antes y después del campamento.

En el período pre campamento no registramos diferencias significativas en los niveles de HbA1c entre niños con y sin cobertura. A los 4 y 7 meses post campamento los valores promedio de $\mathrm{HbA} 1 \mathrm{c}$ de los 37 niños disminuyeron significativamente $(8.8 \pm 1.8 \%$ y $9.5 \pm 2.5 \%$ respectivamente $)$ 
respecto al período previo $(10.3 \pm 2.3 \%, p<0.001)$. No hubo diferencias significativas entre los valores de los 4 y 7 meses post campamento.

Los valores promedio de HbA1c de los niños que asistieron al EF y tenían cobertura descendieron significativamente a los $4(p<0.003)$ y a los 7 meses $(p<0.002)$ post campamento (Tabla 4). Esta diferencia no se registró en los niños sin cobertura.

En el grupo de niños que no asistió al EF y tenían cobertura también registramos descensos significativos de los valores de $\mathrm{HbA} 1 \mathrm{c}$ tanto a los 4 (8.3 $\pm 1.7 \%, p<0.001)$ como a los 7 meses $(9.1 \pm 2.1 \%, p<0.01)$ post campamento respecto de los valores pre campamento.

Aunque en el período previo al CND los valores promedio de $\mathrm{HbA} 1 \mathrm{c}$ no mostraron relación con la cobertura de salud ni con el nivel de educación de sus familiares, a los 4 y 7 meses post campamento los niños con cobertura presentaron mejor control metabólico que los sin cobertura $(7.9 \pm 1.4 \%$ vs. 10.1 $\pm 1.5 \%, p<0.001)$ y $(8.2 \pm 1.8 \%$ vs. $11.5 \pm 2.1 \%, p<0.001)$ respectivamente. También encontramos diferencias significativas en los valores de $\mathrm{HbA} 1 \mathrm{c}$ de los niños con familiares que poseian nivel terciario o universitario vs. los que tenían nivel primario completo o incompleto: $7.6 \pm 1.3 \%$ vs. $9.8 \pm 1.4 \%, p<0.005$ a los 4 meses post campamento. Similares resultados encontramos a los 7 meses post campamento: $7.8 \pm 1.6 \%$ vs. $11.4 \pm 2.4 \%, p<0.005$.

En el período pre campamento solo el 16\% (n:6) de los niños tenía valores de $\mathrm{HbA} 1 \mathrm{c} \leq 8 \%$. Este porcentaje aumentó significativamente a $40 \%$ (n:15) a los 4 meses $(p<0.05)$, de los cuales el 93\% (n:14) tenían cobertura y a $30 \%(\mathrm{n}: 11)$ a los 7 meses post campamento (100\% con cobertura). 
El mejor control metabólico a los 4 y 7 meses post campamento se observó en el grupo de niños con cobertura que participó de las dos intervenciones educativas (CND y EF), con valores promedio de $7.4 \pm 0.9 \%$ y de $7.2 \pm 1.0 \%$ vs. $9.1 \pm 1.9 \%$ (pre campamento), $p<0.003$ y $p<0.002$ respectivamente. Elegimos el umbral de $\mathrm{HbA} 1 \mathrm{c}$ de $8.0 \%$ como una meta realista en este grupo etáreo, considerando que por encima de este valor el riesgo de desarrollar complicaciones aumenta marcadamente $(7,69,70)$.

Control metabólico y variables del tratamiento:

En la Tabla 5 se relacionan la frecuencia diaria de automonitoreo glucémico con el número de inyecciones diarias y los valores promedio de $\mathrm{HbA1c}$ post campamento.

TABLA 5. Frecuencia diaria de AMG, número de inyecciones diarias y control metabólico post intervención

\begin{tabular}{|c|c|c|c|c|c|}
\hline $\begin{array}{c}N^{\circ} \text { de } \\
\text { AMG }\end{array}$ & $\begin{array}{c}\text { Número de } \\
\text { niños sin } \\
\text { cobertura } \\
\text { (n:15) }\end{array}$ & $\begin{array}{c}\text { Número de } \\
\text { niños con } \\
\text { cobertura } \\
(\mathrm{n}: 22)\end{array}$ & $\begin{array}{c}\text { Número de } \\
\text { Inyecciones/día }\end{array}$ & $\begin{array}{c}\text { HbA1c (\%) } \\
4 \text { meses } \\
\text { Post } \\
\text { Campamento }\end{array}$ & $\begin{array}{c}\text { HbA1c (\%) } \\
7 \text { meses } \\
\text { Post } \\
\text { Campamento }\end{array}$ \\
\hline $\mathbf{1}$ & 3 & - & $1 \pm 1$ & $10.9 \pm 1.1$ & $11.7 \pm 2.1$ \\
\hline $\mathbf{2}$ & 7 & 4 & $2 \pm 0.4$ & $9.3 \pm 2.1$ & $10.8 \pm 2.7$ \\
\hline $\mathbf{3}$ & 4 & 5 & $2 \pm 1$ & $8.3 \pm 1.4$ & $9.5 \pm 2.7$ \\
\hline $\mathbf{2 4}$ & 1 & 13 & $3 \pm 1$ & $8.2 \pm 1.5$ & $8.0 \pm 1.6$ \\
\hline $\begin{array}{c}\text { Valor } \\
\text { de P }\end{array}$ & - & - & 0.006 & 0.006 & $<0.001$ \\
\hline
\end{tabular}

AMG: automonitoreo glucémico

Los resultados se expresan como media $\pm D E$ (desvío estándar) y frecuencia

Los valores promedio de $\mathrm{HbA} 1 \mathrm{c}>9 \%$, correspondieron a niños que se aplicaban <2 inyecciones diarias de insulina y realizaban entre 1-2 
determinaciones de glucemia por día; el $66 \%$ de ellos eran niños sin cobertura. El $82 \%$ de los niños con cobertura y el $33 \%$ de los sin cobertura $(p<0.001)$ realizaban 3 ó más determinaciones glucémicas diarias y se aplicaban más de 2 inyecciones/día. Los valores promedio de $\mathrm{HbA} 1 \mathrm{c}$ de este último grupo fueron significativamente menores que los del primero $(8.2 \pm 1.5 \%$ vs. $9.6 \pm 2 \%, p$ $<0.005)$ a los 4 meses y de $(8.6 \pm 2.1 \%$ vs. $10.9 \pm 2.5 \%, p<0.001)$ a los 7 meses post campamento. Observamos los valores más bajos de HbA1c a los 4 y 7 meses post campamento en niños que realizaban 4 o más determinaciones de glucemia diarias ( $59 \%$ con cobertura). 
El niño con diabetes debe afrontar numerosas responsabilidades y tomar decisiones que otros niños de la misma edad no deben enfrentar. Para hacerlo exitosamente, él y su familia necesitan adquirir conocimientos, autodisciplina y habilidades para la resolución de problemas a través de un proceso de educación continuo, adaptado a la edad y madurez del niño como así también a las características socioculturales de su entorno.

Este estudio representa la primera evaluación prospectiva del efecto de un programa de educación terapéutica desarrollado durante un campamento y encuentro familiar para niños con diabetes tipo 1 y sus familiares, organizado por el CENEXA, sobre conocimientos, habilidades e indicadores clínicometabólicos.

La literatura plantea que la experiencia recreativa y educativa del campamento se debe desarrollar en un ambiente seguro para el niño $(45,61)$. Dicho ambiente debe proveer un plan de alimentación, de actividad física, de monitoreo glucémico frecuente y de inyecciones de insulina adecuado, supervisado por un equipo interdisciplinario entrenado que asegure un control metabólico eficaz. Estas condiciones se cumplieron en nuestro campamento ya que, aunque la optimización del control glucémico durante el campamento no es su objetivo principal, el valor promedio diario de glucemias fue significativamente menor al registrado en las semanas anterior y posterior al mismo y no se observaron descompensaciones metabólicas graves. Como ocurre frecuentemente, la disminución de la glucemia se acompañó de un incremento en el número de hipoglucemias leves a moderadas de origen multicausal: 
1) frecuencia e intensidad de la actividad física durante el CND mayor que la practicada por los niños en su vida cotidiana fuera del ámbito escolar;

2) ajustes diarios permanentes en la ingesta de carbohidratos y en la dosis de insulina (de acción rápida y prolongada) supervisados por integrantes del equipo de campamento y

3) posible sub-registro de episodios de hipoglucemia en los períodos pre y post campamento (planillas de registro solicitadas).

En este sentido, en su documento de consenso acerca del control de la diabetes durante los campamentos, la Asociación Americana de Diabetes (ADA) recomienda continuar durante el campamento con el régimen de insulinoterapia previa al mismo (61). Sin embargo, tanto ésta como otras publicaciones sobre el tema $(49,59)$, aconsejan disminuir la dosis de insulina previa desde el inicio del campamento en los niños con buen control metabólico y escaso desarrollo de actividad física, para mantener la glucemia dentro de límites de seguridad. En nuestro caso tanto en el CND como en el EF, no disminuimos las dosis de insulina al ingreso, sino que las modificamos individualmente de acuerdo a las actividades planificadas, a los valores del automonitoreo glucémico diarios y a la respuesta metabólica de las jornadas anteriores. La resultante final fue la mencionada mejoría del control glucémico, comparado con el de las semanas previa y posterior y la prevención de episodios de hipoglucemia severa o de cetoacidosis.

En el campamento, el predominio de la diversión, la cooperación entre pares y adultos que comparten la misma afección y el empleo de estrategias educativas adecuadas a la edad y características de los niños, promueven un intercambio altamente motivador para que el niño adquiera 
conocimientos y habilidades para el manejo diario de su enfermedad. En nuestro estudio, verificamos cambios importantes en el área cognitiva y en el de las habilidades que se mantuvieron en el período post campamento. Los niños aumentaron significativamente sus conocimientos sobre la diabetes y su tratamiento y adquirieron y perfeccionaron habilidades para el manejo de técnicas de autocuidado, independientemente de la edad y duración de su enfermedad.

Diversos autores obtuvieron resultados similares $(50,52,54,58,59)$ y uno de ellos (54) demostró que independientemente de la edad del niño, la probabilidad de que las habilidades necesarias para un autocuidado efectivo se pongan en práctica aumenta, si hay una base adecuada de conocimientos sobre la enfermedad.

Durante el CND, el grupo de niños que utilizaba el método de comparación visual para el monitoreo habitual de su glucemia y aquellos con posibles dificultades para realizar una alimentación balanceada por su situación socioeconómica, participaron de sesiones educativas individuales además de las grupales. Sin embargo, nuestros resultados demuestran que aún cuando el programa educativo empleado logró incrementar en ellos conocimientos y habilidades, estos cambios, no se reflejaron en una mejoría de su control metabólico luego del CND.

Frente a estos resultados, cabe preguntarse si la intervención educativa implementada fue la más apropiada para este grupo de niños en particular. Igualmente si existen barreras para lograr el éxito de la educación terapéutica. 
Si bien el diseño educativo empleado durante el CND y el EF no permite contestar con precisión la primera pregunta, los cambios observados en el área cognitiva y de las habilidades sugieren que, al menos en parte, las técnicas educativas empleadas lograron resultados favorables. De igual manera verificamos que los valores promedio de $\mathrm{HbA} 1 \mathrm{c}$ iniciales mejoraron en el período post campamento. En efecto los valores promedio de $\mathrm{HbA} 1 \mathrm{c}$ de los niños previos al CND eran de $10 \pm 2 \%$, demostrativos de un control metabólico deficiente según los estándares de la Asociación Latinoamericana de Diabetes (71). Estos valores los exponen a un mayor riesgo de desarrollo precoz de complicaciones micro y macroavasculares $(7,10,72)$. A los 4 meses post campamento la $\mathrm{HbA} 1 \mathrm{c}$ descendió en el $40 \%$ de los niños participantes y en el $30 \%$ a los 7 meses, indicando una franca mejoría del control metabólico (HbA1c $\leq 8 \%)$. En ambos casos sin embargo, la mejoría estuvo condicionada por la cobertura de salud ( $93 \%$ y $100 \%$ respectivamente). Cabe agregar que no existe hasta la fecha ninguna publicación en la que se comparen los efectos a corto y mediano plazo de un programa educativo dirigido a niños con diabetes desarrollado durante un campamento y los obtenidos luego de un programa de educación convencional. Esta comparación es necesaria para determinar si la experiencia recreativa y de camaradería del campamento es más eficiente para lograr los objetivos de la educación terapéutica.

Respecto al segundo interrogante, hay evidencias de la existencia de factores no educativos que condicionan el éxito del programa educativo tales como las características estructurales y funcionales de la familia, carencia o deficiente cobertura de salud, situación socioeconómica y hábitos culturales y alimentarios $(38,73-76)$. En nuestro estudio el descenso de la HbA1c post 
campamento antes mencionado no fue uniforme: mientras el grupo de niños con cobertura, especialmente los que participaron junto a sus familiares del EF, mantuvo los valores de $\mathrm{HbA} 1 \mathrm{c}<8 \%$ hasta el final de la evaluación, el grupo sin cobertura lo aumentó. Por otra parte en la evaluación post campamento, los niños con mayor frecuencia de automonitoreo glucémico y con aplicación de más de 2 inyecciones de insulina diarias, tuvieron mejor control metabólico sostenido hasta el final del estudio. Cerca del $80 \%$ de ellos poseía cobertura de salud que le aseguraba la provisión del material necesario para llevar a cabo este tipo de tratamiento. En conjunto, estos datos sugieren que:

- el factor económico per se no asegura un mejor control metabólico ya que antes del CND los valores de $\mathrm{HbA} 1 \mathrm{c}$ eran elevados y similares en niños con y sin cobertura (Tabla 4),

- la educación recibida durante el CND, evaluada a través del incremento de conocimientos y habilidades, disminuyó los valores de $\mathrm{HbA1c}$ a valores considerados adecuados para las metas terapéuticas de niños con diabetes tipo 1; el hecho sugiere que la educación optimizó el uso de los recursos terapéuticos disponibles. Sin embargo este descenso atribuido a la educación estuvo aparentemente condicionado por la accesibilidad de los niños a la atención médica, medicamentos e insumos requeridos para su control metabólico frecuente (valores mayores de Hba1c asociados a insuficiente número de tiras reactivas para automonitoreo glucémico en niños sin cobertura). Para confirmar esta hipótesis deberíamos verificar si la provisión gratuita de más tiras reactivas y glucómetro junto con un programa educativo, aumenta el número de automonitoreos y mejora su control metabólico. 
Nuestros resultados también confirman lo planteado por otros autores respecto a, que conocer más acerca de la diabetes y adquirir habilidades para ejecutar técnicas de tratamiento y autocuidado diario, no implican necesariamente un cambio de actitud del paciente, ni se correlacionan con un mejor control metabólico $(12,77)$. Al igual que nosotros, Wolanski y col. estudiaron el efecto de una intervención educativa individualizada sobre el manejo y precisión de la técnica de automonitoreo glucémico en un grupo de niños y adolescentes con diabetes que participaron en un campamento de verano. Los autores concluyeron que los errores cometidos por los niños al emplear esta técnica luego del programa educativo, se debieron más a una motivación deficiente que a una falta de conocimientos y habilidades para su ejecución (58). En consecuencia, para lograr sus objetivos la educación terapéutica deberá motivar, incrementar conocimientos diabetológicos, desarrollar habilidades técnicas, reiterarse y acompañarse de un acceso adecuado a medicamentos e insumos.

Numerosas evidencias descriptas en la literatura refuerzan nuestras conclusiones. Así por ejemplo el éxito obtenido en el DCCT (8) y en otros estudios con el tratamiento intensificado $(16,78)$ se debió en gran parte a su combinación con la educación continuada de sus participantes por un equipo interdisciplinario experimentado. Más afín con nuestro modelo educativo, Karaguzel y col. demostraron que la implementación simultánea durante un campamento de un régimen intensificado de insulina y de un programa educativo, disminuyeron los valores de $\mathrm{HbA} 1 \mathrm{c}$ a los 6 y 12 meses post campamento (48). 
Aunque escasos y mayoritariamente descriptivos, otros estudios sobre campamentos de niños con diabetes demostraron una mejoría del control metabólico a corto y mediano plazo $(46,48,52,56,60)$. Así por ejemplo Koplatadze y col (56), demostraron una disminución significativa de la HbA1c a los 3 meses post campamento, que fue más pronunciada en niños que tenían pobre control metabólico previo al campamento. Este resultado fue independiente de la frecuencia del automonitoreo glucémico. Por el contrario, Haller y col. demostraron que en un grupo de 229 niños y adolescentes, los valores de HbA1c pre campamento más bajos se correlacionaron significativamente con la frecuencia del automonitoreo glucémico (47). Esta asociación frecuencia de automonitoreo glucémico y niveles de $\mathrm{HbA} 1 \mathrm{c}$ se ha estudiado extensivamente fuera del contexto de los campamentos confirmándose en varios de ellos la coincidencia de mayor frecuencia de determinaciones de glucemia con la reducción de HbA1c (79-81). En consecuencia, el automonitoreo glucémico es una herramienta imprescindible para alcanzar y mantener objetivos glucémicos específicos en todo paciente con insulinoterapia intensificada. En el caso de los niños con diabetes tipo 1 son necesarias 4 o más determinaciones glucémicas diarias para determinar patrones de hipoglucemia e hiperglucemia y proveer información para realizar ajustes en la dosis de insulina $(29,82)$. Cabe aclarar sin embargo que el número de determinaciones glucémicas o de inyecciones de insulina per se, no serán suficientes para mejorar el control metabólico, a menos que el niño y su familia hayan aprendido a traducir sus resultados en ajustes apropiados en las dosis de insulina, en la ingesta de carbohidratos y en la carga de actividad física $(22,83,84)$. 
Probablemente las variables consideradas no sean las únicas que condicionan el logro de las metas terapéuticas propuestas: una familia sin cobertura, implica asimismo otras características como desempleo o subempleo de los progenitores, insuficiente preparación académica o técnica para acceder a un empleo, menor ingreso para las necesidades de alimentación balanceada, traslado y educación.

En conclusión, nuestro estudio muestra que un programa educativo implementado durante un campamento para niños con diabetes seguido de un encuentro familiar aumentó los conocimientos y habilidades de los participantes, facilitó la utilización de un régimen de automonitoreo glucémico y de insulinoterapia intensificados y disminuyó los niveles de HbA1c post campamento. La persistencia a mediano plazo de estos efectos beneficiosos dependió en parte de la continuidad de la educación y del grado de accesibilidad de los niños a una atención, medicamentos e insumos adecuada. Esto demuestra que factores socioculturales y económicos condicionan la efectividad de los resultados a mediano plazo de la educación terapéutica. Cabe entonces acordar con la Federación Internacional de Diabetes en su sección consultiva sobre diabetes en la infancia y adolescencia, que dice: "Todos los niños y jóvenes con diabetes tienen derecho a un control médico competente y a una educación diabetológica impartida por un equipo con experiencia en el tema y que comprenda las necesidades médicas y psicosociales de los jóvenes y sus familias. Dado que el niño no puede luchar por estos derechos, es responsabilidad de la sociedad proporcionar todo el apoyo necesario al niño y su familia. Ellos deberían contar con recursos y esfuerzos médicos, sociales, públicos, gubernamentales e industriales". 
1. American Diabetes Association. Diagnosis and Classification of Diabetes Mellitus (position statement). Diabetes Care 2006;29:S43-48

2. Gagliardino JJ, Olivera EM, Etchegoyen GS, González Claudio, Guidi ML. Evaluación y costos del proceso de atención de pacientes diabéticos. Medicina 2000;60:880-888.

3. Brandle M, Honghong Z, Smith BRK, Marriott D, Burke R, et al. The direct medical cost of type 2 diabetes. Diabetes Care 2003;26:2300-2304.

4. Walsh MG, Zgibor J, Songer T, Borch-Johnsen K, Orchard TJ, DiaComp Investigators. The socioeconomic correlates of global complication prevalence in type 1 diabetes (T1D): a multinational comparison. Diabetes Res Clin Pract. 2005;70(2):143-50.

5. Gagliardino JJ y col. Control y prevención secundaria para personas con diabetes: impacto de la implementación de un programa de atención (PROPAT). Rev Soc Arg de Diabetes. 2001;35:N²:77-86.

6. Gagliardino JJ, de la Hera M, Siri F. Evaluación preliminar de la calidad de atención de personas con diabetes en Argentina. Rev Soc Arg de Diabetes. $2001 ; 35(3): 1221-1333$.

7. The Diabetes Control and Complications Trial Research Group. The relationship of glycemic exposure $(\mathrm{HbA} 1 \mathrm{c})$ to the risk of development and progression of retinopathy in the Diabetes Control and Complications Trial. Diabetes 1995;44:968-983.

8. The Diabetes Control and Complications Trial Research Group. The effect of intensive treatment of diabetes on the development and progression of longterm complications in insulin-dependent diabetes mellitus. $N$ Engl J Med. 1993;329:977-986. 
9. UK Prospective Diabetes Study (UKPDS) Group. Effect of intensive bloodglucose control with metformin on complications in overweight patients with type 2 diabetes (UKPDS 34). Lancet. 1998;352:854-865.

10. The Epidemiology of Diabetes Interventions and Complications Research Group. Sustained effect of intensive treatment of type 1 diabetes mellitus on development and progression of diabetic nephropathy: the Epidemiology of Diabetes Interventions and Complications (EDIC) study. Jama 2003;290: $2159-67$.

11. Assal JP, Mühlhauser I, Pernet A, Geller R, Jorgens V, Berger M. Patient education as the basis for diabetes care in clinical practice and research. Diabetologia 1985;28:602-613.

12. Korhonen $\mathrm{T}$, Huttunen JK, Aro A, Hentinen $\mathrm{M}$, Ihalainen $\mathrm{O}$, Majander $\mathrm{H}$, Siitonen O, Uusitupa M, Pyorala K. A controlled trial on the effects of patient education in the treatment of insulin-dependent diabetes. Diabetes Care $1983 ; 6$ (issue 3):256-261.

13. Rosenquist U, Theman J, Assal JP (Diabetes Education Study Group of the EASD). Nuevos avances en educación diabetológica: la necesidad de un nuevo rol del paciente, del enfoque clásico al holístico. Rev Soc Arg de Diabetes. 1995;29 (4):127-138.

14. Clement S. Diabetes self-management education. Diabetes Care 1995;18:1204-14.

15. Terent A, Hagfall $O$, Cederholm $U$. The effect of education and selfmonitoring of blood glucose on glycosylated hemoglobin in type 1 diabetes. A controlled 18-month trial in a representative population. Acta Med Scand. $1985 ; 217: 47-53$ 
16. Mühlhauser I, Bruckner I, Berger M, Cheta D, Jorgens V, Ionescu-Tirgoviste $C$ et al. Evaluation of an intensified insulin treatment and teaching programme as a routine management of type 1 (insulin-dependent) diabetes. The Bucharest-Düsseldorf Study. Diabetologia 1987;30:681-90.

17. Kronsbein P, Jorgens V, Mühlhauser I, Scholz V, Venhaus A, Berger M. Evaluation of a structured treatment and teaching programme on noninsulin-dependent diabetes. Lancet 1988;2:1407-11.

18. Domenech MI, Assad D, Mazzei ME, Kronsbein P, Gagliardino JJ. Evaluation of the effectiveness of an ambulatory teaching/treatment programme for non-insulin dependent (type 2) diabetic patients. Acta Diabetol. 1995;32:143-7.

19. Assad D, Domenech MI, Mercuri N, y col. Programa de educación del diabético insulino dependiente: primera evaluación de sus resultados en el Centro Bernardo Houssay. Rev Soc Arg de Diabetes. 1995;29:144-151.

20. Assal JP, Jacquemet S, Morel Y. The added value of therapy in diabetes: the education of patients for self-management of their disease. Metabolism 1997;46 (12 Suppl 1):61-64.

21. Assal JP. Primary prevention of NIDDM: a future dream. Diabetes Metab Rev. 1997;13:113-117.

22. Dorchy H, Roggemans MP, Willems D. Glycated Hemaglobin and related factors in diabetic children and adolescents under 18 years of age: a Belgian experience. Diabetes Care 1997;20:2-6.

23. Gagliardino JJ, Etchegoyen G. A model educational program for people with type 2 diabetes: a cooperative Latin American implementation study (PEDNID-LA). Diabetes Care 2001;24:1001-1007. 
24. Norris SL, Lau J, Smith SJ, Schmid CH, Engelgau MM. Self-mangement education for adults with type 2 diabetes: a meta-analysis of the effect on glycemic control. Diabetes Care 2002;25:1159-1171.

25. Delamater AM, Jacobson AM, Anderson B, Cox D, Fisher L, et al. Psychosocial therapies in diabetes. Diabetes Care 2001;24:1286-1292.

26. Pouwer F, Snoek FJ, van der Ploeg HM, Ader HJ, Heine RJ. Monitoring of psychological well-being in outpatients with diabetes: effects on mood, $\mathrm{HbA}$ (1c), and the patients evaluation of the quality of diabetes care: a randomized controlled trial. Diabetes Care 2001;24:1929-1935.

27. Skovlund SE, Peyrot M on the behalf of the DAWN International Advisory Panel. The Diabetes Attitudes, Wishes and Needs (DAWN) Program: A new approach to improving outcomes in diabetes care. Diabetes Spectr. 2005;18:136-142.

28. Providing education for children and adolescents with type 1 or 2 diabetes mellitus. In: International Consensus: Position Statements for Diabetes Education. London: International Diabetes Federation; 2000:25-34.

29. Silverstein J, Klingensmith G, Copeland K, Plotnick L, Kaufman F, et al. Care of children and adolescents with type 1 diabetes: a statement of the American Diabetes Association. Diabetes Care 2005;28:186-212.

30. Schafer LC, McCaul KD, Glasgow RE. Supportive and nonsupportive family behaviors: relationships to adherence and metabolic control in persons with type I diabetes. Diabetes Care 1986;9:179-185.

31. Laffel LM, Vangsness L, Connel A, Goebel-Fabbri A, Butler D, Anderson BJ. Impact of ambulatory, family-focused teamwork intervention on glycemic control in youth with type 1 diabetes. J Pediatr. 2003;142 (4):409-16. 
32. American Diabetes Association. Diabetes Care in the school and day care setting. Diabetes Care 2004;27 (1):S122-S128.

33. Lewin AB, Heidgerken AD, Geffken GR, Williams LB, Storch EA et al. The relation between family factors and metabolic control: the role of diabetes adherence. Journal of Pediatric Psychology 2006;31(2):174-183.

34. Tubiana-Rufi N, Moret L, Czernichow P, Chwalow J, The PEDIAB Collaborative Group. Risk factors for poor glycemic control in diabetic children in France. Diabetes Care 1995;18:1479-1482

35. Madden PB. Children and families living with diabetes. Diabetes Spectrum $2004 ; 17: 18-21$

36. Kovacs M, Kass RE, Schenell TM, Goldston D, Marsh J. Family functioning and metabolic control of school-aged children with IDDM. Diabetes Care $1989 ; 12: 409-415$

37. Grey M, Cameron ME, Lipman TH, Thurber FW. Psychosocial status of children with diabetes in the first 2 years after diagnosis. Diabetes Care 1995; 18:1330-1336.

38. Rosilio M, Cotton JB, Wieliczko MC, Gendrault B, Carel JC, Couvaras O, Ser $\mathrm{N}$ et al. Factors associated with glycemic control: A cross-sectional nationwide study in 2579 french children with type 1 diabetes. Diabetes Care 1998:21:1146-1153.

39. Guthrie DW, Bartsocas $\mathrm{CH}$, Jarosz-Chabot $\mathrm{P}$, Konstantinova $\mathrm{M}$. Psychosocial issues for children and adolescents with diabetes: overview and recommendations. Diabetes Spectrum 2003;16:7-12.

40. Follansbee DS. Assuming responsibility for diabetes management: what age? What price? The Diabetes Educator 1989;15 (4):347-53. 
41. Ingersoll GM, Orr DP, Herrold AJ, Golden MP. Cognitive maturity and selfmanagement among adolescents with insulin-dependent diabetes mellitus. $J$ Pediatr. 1986;108(4):620-623.

42. Wysocki T, Meinhold PA, Abrams KC, Barnard MU, Clarke WL et al. Parental and professional estimates of self-care independence of children and adolescents with IDDM. Diabetes Care 1992;15:43-52.

43. Anderson BJ, Auslander WF, Jung KC, Miller JP, Santiago JV. Assessing family sharing of diabetes responsibilities. J Pediatr Psychol. 1990;15:477492.

44. Anderson BJ, Brackett J, Ho J, Laffel LMB. An office-based intervention to maintain parent-adolescent teamwork in diabetes management. Diabetes Care 1999;22:713-721.

45. ISPAD Consensus Guidelines for the Management of Type 1 Diabetes Mellitus in Children and Adolescents. International Society for Pediatric and Adolescent Diabetes. Ed. PGF Swift. Publ. Medforum, Zeist, Netherlands; 2000

46. Santiprabhob J, Likitmaskul S, Sriwijitkamol A, Peerapatdit T, Sawathiparnich A, et al. Improved glycemic control among Thai children and young adults with type 1 diabetes participating in the diabetes camp. J Med Assoc Thai. 2005;88 (8):S38-S42.

47. Haller MJ, Stalvey MS, Silverstein JH. Predictors of control of diabetes: monitoring may be the key. The Journal of Pediatrics 2004;144(5):660-661.

48. Karagüzel G, Bircan I, Erişir S, Bundak R. Metabolic control and educational status in children with type 1 diabetes: effects of a summer camp and intensive insulin treatment. Acta Diabetol. 2005;42:156-161. 
49. Braatvedt GD, Mildenhall L, Patten C, Harris G. Insulin requirements and metabolic control in children with diabetes mellitus attending a summer camp. Diabet Med. 1997;14: 258-61.

50. Santos de Rodriguez M. Campamento de verano para niños con diabetes mellitus realizado en Monterrey, N.L. México. Salud Pública de México. $1985 ; 27: 332-335$

51. Mimura G. Summer camp. Diabetes Res Clin Pract. 1994;24 (suppl ):S287S290.

52. Misuraca A, Di Gennaro M, Lioniello M, Duval M, Aloi G. Summer camps for diabetic children: an experience in Campania, Italy. Diabetes Res Clin Pract. $1996 ; 32: 91-96$

53. Kanji I, Mitsuru H, Shotaro $\mathrm{K}$ y col. Glycemic control, growth and complications in children with insulin-dependent diabetes mellitus-a study of children enrolled in a Summer camp program for diabetics in Kinki district, Japan. Diabetes Research and Clinical Practice 1995;(28):185-190.

54. Christensen Karen S. Self-management in diabetic children. Diabetes Care 1983;(6):552-555.

55. Bohm P, Ceschel S, Capila MT et al. Blood sugar control in children and young diabetics during an educational summer camp. Pediatr Med Chir. $1997 ; 19(6): 447-9$

56. Koplatadze K, Koplatadza M, Kacharava L et al. Diabetes camps: an international experience. Mo Med. 2003;100(2):145-7.

57. Post EM, Moore JD, Ihrke J et al. Fructosamine levels demonstrate improved glycemic control for some children attending a diabetes summer camp. Pediatric Diabetes 2000;1(4):204. 
58. Wolanski R, Sigman T, Polychronakos C. Assessment of blood glucose selfmonitoring skills in a camp for diabetic children: the effect of individualized feedback counselling. Patient Education and Counselling 1996;29:5-11.

59. Semiz S, Bilgin ÜO, Bundak R, Bircan I. Summer camps for diabetic children: an experience in Antalya, Turkey. Acta Diabetol. 2000;37:197-200.

60. Strickland $\mathrm{AL}, \mathrm{McF}$ arland $\mathrm{KF}$, Murtiashaw $\mathrm{MH}$ et al. Changes in blood protein glycosylation during a diabetes summer camp. Diabetes Care 1984; 7(2):183-185.

61. American Diabetes Association. Diabetes Care at Diabetes Camps. Diabetes Care 2004;27:S129-S131.

62. Plante WA, Lobato D, Engel R. Review of group interventions for pediatric chronic conditions. J Pediatr Psychol. 2001;26:435-453.

63. Rickheim PL, Weaver TW, Flader JL, Kendall DM. Assessement of group versus individual diabetes education. Diabetes Care 2002;25:269-274

64. Mensing CR, Norris SL. Group education in diabetes: effectiveness and implementation. Diabetes Spectrum 2003;16:96-103.

65. Mannucci E, Pala L, Rotella CM. Long-term interactive group education for type 1 diabetic patients. Acta Diabetol. 2005;42:1-6.

66. Torresani ME. Cuidado nutricional pediátrico. EUDEBA, Buenos Aires, 2001.

67. Lejarraga H, Orfila G. Estándares de peso y estatura para niñas y niños argentinos desde el nacimiento hasta la madurez. Arch.Argent.Pediatr. $1987 ; 85-209$. 
68. Rolland-Cachera M, Cole T, Sempe J, Tichet C, Rossignol C, A Charaud A. Body mass index variations: centiles from birth to 87 years. Eur J Clin Nutr. $1991 ; 45: 13-21$.

69. Krolewski AS, Laffel LMB, Krolewski M, Quinn M, Warram JH. Glycosylated hemoglobin and the risk of microalbuminuria in patients with insulindependent diabetes mellitus. N Engl J Med. 1995;332:1251-1255.

70. Mortensen HB, Hougaard P, Hvidore Study Group on Chilhood Diabetes. Comparison of metabolic control in a cross-sectional study of 2,873 children and adolescents with IDDM from 18 countries. Diabetes Care 1997;20:714720.

71. Grupo de Estudio Latino Americano sobre diabetes en el Niño y el Adolescente (GELADNA). Diagnóstico y tratamiento de la diabetes mellitus en el niño y el adolescente. Revista de la Asociación Latinoamericana de Diabetes 2005; Vol XIII, N³:98-114.

72. Díaz El, Pérez Aguilar RC, Velarde MS, Prado MM, Carrizo TR y col. Parámetros de la hemostasia en una población infanto-juvenil con diabetes tipo 1 de San Miguel de Tucumán, Argentina. Rev Soc Arg de Diabetes. $2005 ; 39(3): 15-21$

73. Lewin AB, Heidgerken AD, Geffken GR, Williams LB, Storch EA et al. The relation between family factors and metabolic control: the role of diabetes adherence. Journal of Pediatric Psychology 2006;31(2):174-183.

74. Tubiana-Rufi N, Moret L, Czernichow P, Chwalow J, The PEDIAB Collaborative Group. Risk factors for poor glycemic control in diabetic children in France. Diabetes Care 1995;18:1479-1482 
75. Delamater AM, Albrecht DR, Postellon DC, Gutai JP. Racial differences in metabolic control of children and adolescents with type 1 diabetes mellitus. Diabetes Care 1991;14:20-25.

76. Gallegos-Macias AR, Macias SR, Kaufman E, Skipper B, Kalishman N. Relationship between glycemic control, ethnicity and socioeconomic status in Hispanic and white non-Hispanic youths with type 1 diabetes mellitus. Pediatr Diabetes. 2003;4(1):19-23.

77. Benney LJ, Dunn SM: Knowledge improvement and metabolic control in diabetes education: approaching the limits? Patient Ed Counsel. 1990; $16: 217-29$.

78. Starostina EG, Antsiferov M, Galstyan GR, Trautner C, Jorgens V, Bott U et al. Effectiveness and cost-benefit analysis of intensive treatment and teaching programmes for type 1 (insulin-dependent) diabetes mellitus in Moscow- blood glucose versus urine glucose self-monitoring. Diabetologia $1994 ; 37: 170-6$.

79. Karter AJ, Ackerson LM, Darbinian JA, D'Agostino RB, Ferrara A et al. Selfmonitoring of blood glucose levels and glycemic control: the Northen California Kaiser Permanente Diabetes Registry. The American Journal of Medicine 2001; Vol 111:1-9.

80. Nyomba BL, Berard L, Murphy LJ. Facilitating access to glucometer reagents increases blood glucose self-monitoring frequency and improves glycemic control: a prospective study in insulin-treated diabetic patients. Diabet Med. 2004;21(2):129-35. 
81. Evans JMM et al. Frequency of blood glucose monitoring in relation to glycemic control: observational study with diabetes database. BMJ $1999 ; 319: 83-86$.

82. American Diabetes Association: Self-monitoring of blood glucose (Position Statement). Diabetes Care 2004:27 (Suppl.1):S91-S93.

83. Delamater AM, Bubb J., Davis SG, Smith JA, Schmidt L, White NH et al. Randomized prospective study of self-management training with newly diagnosed children. Diabetes Care 1990;13:492-498.

84. Mortensen HB, Robertson KJ, Aanstoot HJ, Danne T, Holl RW et al. Insulin management and metabolic control of type 1 diabetes mellitus in childhood and adolescents in 18 countries. Diabetic Medicine 1998;15:752-759. 
TESTIMIONIOS
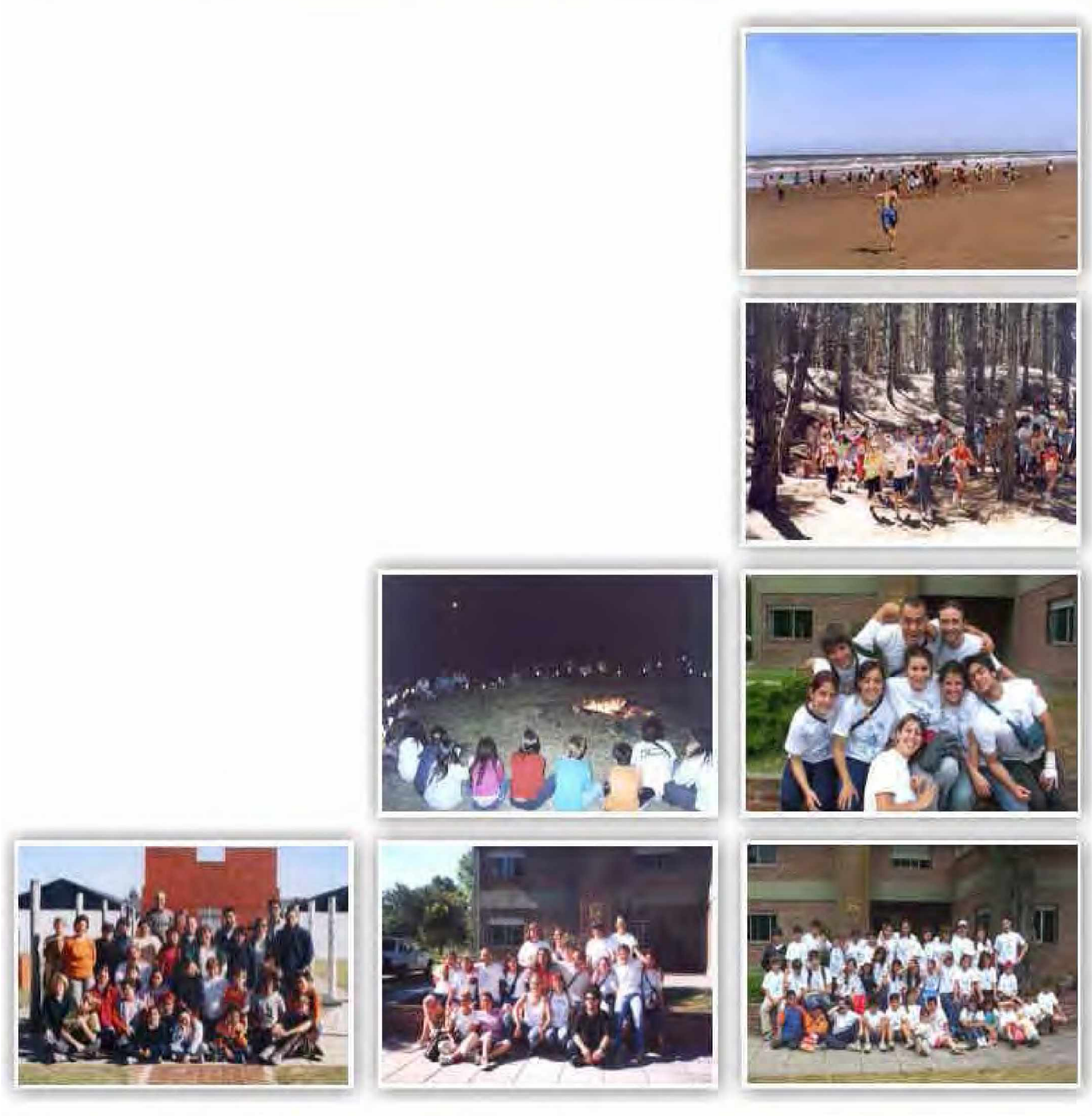
- El camosmeno es un manco ideal donde cada mina debe edsin un gru

lobaco que manemga una

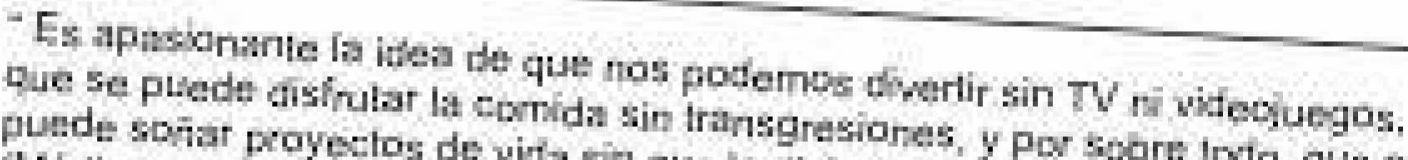
pusde somat proyectos de vida sin transgresionds, y Por soork Irodo, que se (Medies dol compamenlo)

- Si bien la experiecicia comb canoista tue realnente un hecho magico en mi vida ser lloer as lo mas increble y hemoso gue me paso, et compananto es para mí un sosten, un molino y un motor que me da herzas oada vez cue ne siento thise, Sl ver ha carita ue lelicidad que pone cada nene o thitad aprenden a contar

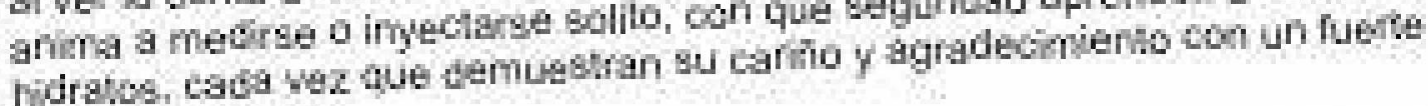
strazo. ILider:

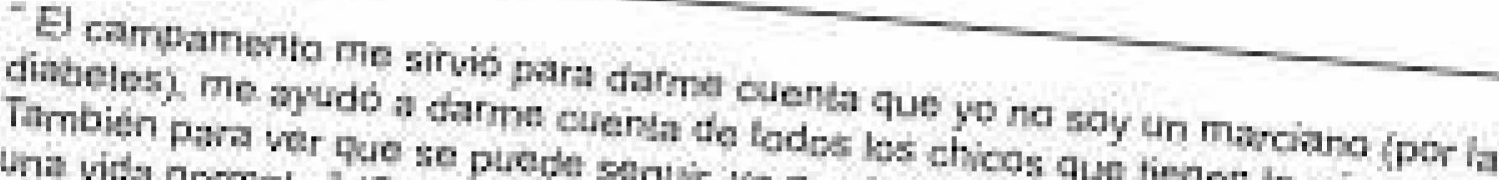

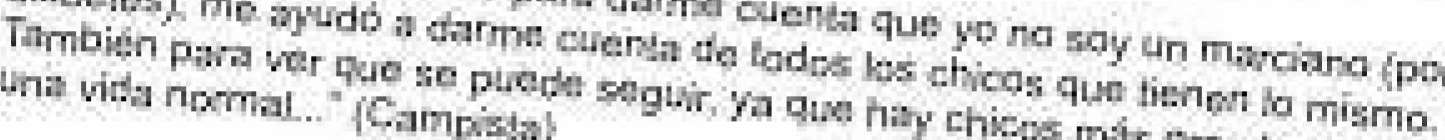

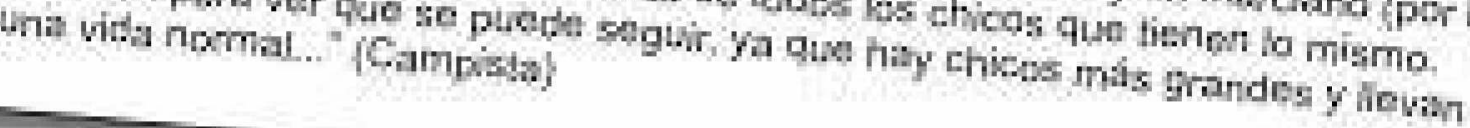

- .comprend ta importamera de la nutricion, apread a respetar la sabd, a abir el coratri ante ias demats personae y a asunir la respongabidad de cudar a un mo babetice... "mand duante el encuentro lamiar)

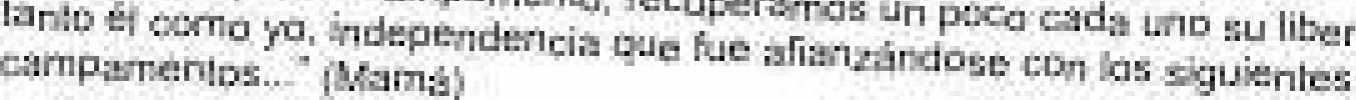


- encona un gupo de padres con una problematica sinilar a la mia, is que inplich una gran byuda.. Imama durante el encuenter faminar

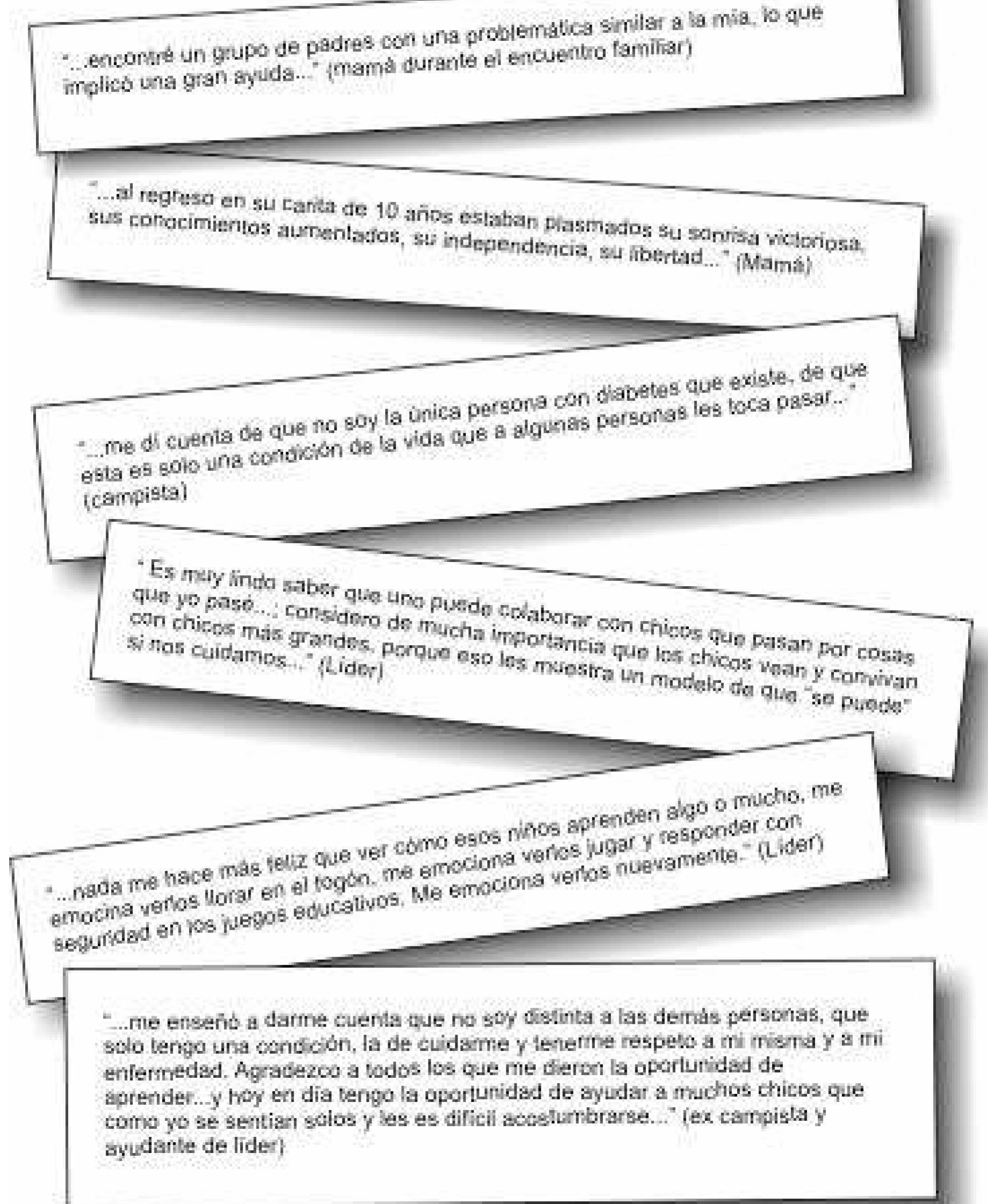




\section{CARTA DE CONSENTIMIENTO INFORMADO}

A quien corresponda:

Por medio de la presente hago constar que he sido informado(a) de los objetivos del Proyecto de investigación que será desarrollado dentro del marco de la Maestría en Educación terapéutica para personas con diabetes y factores de riesgo cardiovascular (CENEXA-Facultad de Ciencias Médicas de la UNLP). El mismo se titula: "Evaluación de un modelo recreativo-educativo para niños con diabetes tipo 1 ".

Por la presente presto $\mathrm{mi}$ consentimiento para la divulgación con fines científicos de los datos registrados de mi hijo/a (en forma anónima) sobre: conocimientos sobre diabetes, habilidades para el automonitoreo glucémico y autoinyección de insulina, indicadores clínicometabólicos y terapéuticos (peso, talla, número de automonitoreos y valores de glucemia, hemoglobina glicosilada, número de inyecciones, tipo y dosis de insulina, rotación y sitios de inyección, plan alimentario) determinados antes, durante y después de finalizado el Campamento para niños con diabetes CENEXA 2004 y el III Encuentro para niños con diabetes y sus familiares.

Asimismo me comprometo a completar las planillas con los datos solicitados por los responsables del Proyecto hasta que finalice el mismo.

Tache lo que no corresponda:

DESEO / NO DESEO conocer los resultados de la investigación.

Atentamente,

FIRMA

DNI

Los datos del firmante corresponden a:
ACLARACION 


\section{CAMPAMENTO PARA NIÑOS CON DIABETES (DÍA TIPO) \\ 12 al 18 de diciembre de 2004 \\ Centro Recreativo San Luis, Aguas Verdes}

\begin{tabular}{|c|c|c|c|c|}
\hline & HORARIO & ACTIVIDADES & EQUIPO ENCARGADO & OBSERVACIONES \\
\hline M & $7: 30$ & $\begin{array}{l}\text { Nos levantamos. } \\
\text { Higiene. } \\
\text { Controles glucemia-glucosuria. Aplicación de insulina }\end{array}$ & Médicos y líderes & \\
\hline$\tilde{N}$ & $9: 00$ & DESAYUNO & Nutrición & \\
\hline A & $\begin{array}{l}9: 45 \\
10: 45 \\
11: 15 \\
12: 00\end{array}$ & $\begin{array}{l}\text { Actividad física. Juegos (en el complejo o en el bosque). } \\
\text { Colación } \\
\text { Educación Diabetológica (juegos educativos, charla teórico-práctica). } \\
\text { Higiene. } \\
\text { Controles glucemia-glucosuria. Eventual aplicación de insulina. }\end{array}$ & $\begin{array}{l}\text { Educ. Física } \\
\text { Nutrición } \\
\text { Nutrición o médico } \\
\text { Médicos y líderes }\end{array}$ & \\
\hline \multirow[b]{2}{*}{$\begin{array}{l}P \\
R \\
E \\
T \\
A \\
R \\
D \\
E\end{array}$} & $13: 00$ & ALMUERZO & Nutrición & \\
\hline & $\begin{array}{l}14: 00 \\
15: 00 \\
16: 30\end{array}$ & $\begin{array}{l}\text { Tiempo libre. Descanso. } \\
\text { Playa o bosque (recreación, juegos). } \\
\text { Colación } \\
\text { Playa o bosque (recreación, juegos). } \\
\text { Regreso. Higiene. } \\
\text { Controles glucemia-glucosuria. Eventual aplicación de insulina. }\end{array}$ & $\begin{array}{l}\text { Educación Fisica } \\
\text { Educación Física } \\
\text { Nutrición } \\
\text { Educación Física } \\
\text { Médicos y líderes }\end{array}$ & \\
\hline \multirow{2}{*}{$\begin{array}{l}\text { T } \\
\text { R } \\
D \\
\text { E }\end{array}$} & $18: 00$ & MERIENDA & Nutrición & \\
\hline & $\begin{array}{l}18: 30 \\
19: 45\end{array}$ & $\begin{array}{l}\text { Juegos grupales. Caminatas. Actividades con la naturaleza. } \\
\text { Higiene: duchas. Orden y limpieza de elementos personales. } \\
\text { Controles glucemia-glucosuria. Aplicación de insulina. }\end{array}$ & $\begin{array}{l}\text { Educación Física } \\
\text { Líderes } \\
\text { Médicos y líderes }\end{array}$ & \\
\hline \multirow{2}{*}{$\begin{array}{l}N \\
O \\
C \\
H \\
E\end{array}$} & $21: 00$ & CENA & Nutrición & \\
\hline & $\begin{array}{l}22: 00 \\
23: 00 \\
23: 30 \\
23: 30\end{array}$ & $\begin{array}{l}\text { Juegos nocturnos. Juegos de salón. Fogón. } \\
\text { Higiene. Eventual control glucemia-glucosuria. Eventual colación. } \\
\text { Nos acostamos. } \\
\text { Reunión de evaluación. }\end{array}$ & $\begin{array}{l}\text { Educación Física } \\
\text { Nutrición, médicos y } \\
\text { líderes }\end{array}$ & \\
\hline
\end{tabular}




\section{ENCUENTRO RECREATIVO Y EDUCATIVO PARA NIÑOS CON DIABETES Y SUS FAMILIARES}

\section{DIA SABADO 16}

LA PLATA, 16 Y 17 DE ABRIL, 2005

\begin{tabular}{|c|c|c|c|c|}
\hline & HORARIO & ACTIVIDADES & $\begin{array}{c}\text { EQUIPO } \\
\text { ENCARGADO } \\
\end{array}$ & OBSERVACIONES \\
\hline $\begin{array}{l}\mathrm{M} \\
\mathrm{A} \\
\tilde{N} \\
\mathrm{~A} \\
\mathrm{~N} \\
\mathrm{~A}\end{array}$ & $\begin{array}{c}8: 00 \\
9: 00 \\
10: 00 \\
10: 30 \\
11: 30\end{array}$ & $\begin{array}{l}\text { Partida desde el Hospital de Niños } \\
\text { Llegada al Camping. Alojamiento. Explicación de actividades y reglas. } \\
\text { Caminata de Reconocimiento / Juegos de integración (adultos y niños ). } \\
\text { Colación } \\
\text { Higiene. } \\
\text { Controles glucemia-glucosuria. Eventual aplicación de insulina. } \\
\text { Talla y peso niños }\end{array}$ & $\begin{array}{l}\text { Todo el Equipo } \\
\text { Educ. Física } \\
\text { Nutrición } \\
\text { Médicos y líderes }\end{array}$ & \\
\hline & $12: 30$ & ALMUERZO & Nutrición & \\
\hline $\begin{array}{l}P \\
R \\
E \\
T \\
A \\
R \\
D \\
E\end{array}$ & $\begin{array}{l}13: 30 \\
14: 00 \\
15: 00 \\
16: 30\end{array}$ & $\begin{array}{l}\text { Tiempo libre } \\
\text { Educación Diabetológica ( juego y charla) (adultos y niños ) } \\
\text { Evaluación conocimientos } \\
\text { Colación. } \\
\text { Gran Juego de Búsqueda (adultos y niños ) } \\
\text { Higiene. } \\
\text { Controles glucemia-glucosuria. Eventual aplicación de insulina. }\end{array}$ & $\begin{array}{l}\text { Educación Física } \\
\text { Nutrición, Médicos } \\
\text { y Líderes. } \\
\text { Nutrición } \\
\text { Educación Física } \\
\text { Médicos y líderes }\end{array}$ & \\
\hline & $17: 30$ & MERIENDA & Nutrición & \\
\hline $\begin{array}{l}T \\
A \\
R \\
D \\
E\end{array}$ & $18: 00$ & $\begin{array}{l}\text { Juegos grupales( niños ). } \\
\text { Educación Diabetológica ( juego y charla) (adultos ) } \\
\text { Higiene. } \\
\text { Controles glucemia-glucosuria. Aplicación de insulina. }\end{array}$ & $\begin{array}{l}\text { Educación Física } \\
\text { Médicos y Líderes } \\
\text { Médicos y líderes }\end{array}$ & \\
\hline $\begin{array}{l}\mathrm{N} \\
\mathrm{O}\end{array}$ & $20: 30$ & CENA & Nutrición & \\
\hline $\begin{array}{l}\mathrm{C} \\
\mathrm{H} \\
\mathrm{E}\end{array}$ & $\begin{array}{l}21: 30 \\
22: 30 \\
23: 00\end{array}$ & $\begin{array}{l}\text { Juegos de salón (adultos y niños) } \\
\text { Higiene. Eventual control glucemia-glucosuria. Eventual colación. } \\
\text { Nos acostamos. }\end{array}$ & $\begin{array}{l}\text { Educación Física } \\
\text { Médicos y Líderes }\end{array}$ & \\
\hline
\end{tabular}


III ENCUENTRO RECREATIVO Y EDUCATIVO PARA NIÑOS CON DIABETES Y SUS FAMILIARES

\section{DIA DOMINGO 17}

LA PLATA, 16 Y 17 DE MAYO, 2005

\begin{tabular}{|c|c|c|c|c|}
\hline & HORARIO & ACTIVIDADES & EQUIPO ENCARGADO & OBSERVACIONES \\
\hline \multirow{3}{*}{$\begin{array}{l}\mathrm{M} \\
\mathrm{A} \\
\tilde{N} \\
\mathrm{~A} \\
\mathrm{~N} \\
\mathrm{~A}\end{array}$} & 8:00 & $\begin{array}{c}\text { Nos levantamos. } \\
\text { Higiene. } \\
\text { Controles glucemia-glucosuria. Aplicación de insulina. }\end{array}$ & $\begin{array}{c}\text { Todo el Equipo } \\
\text { Médicos y Líderes }\end{array}$ & \\
\hline & 9.15 & DESAYUNO & Nutrición & \\
\hline & $\begin{array}{l}10: 00 \\
11: 00 \\
12: 00\end{array}$ & $\begin{array}{c}\text { Educación Diabetológica (juego ) (adultos y niños) } \\
\text { Colación } \\
\text { Clase de Aerobics. } \\
\text { Higiene. } \\
\text { Controles glucemia-glucosuria. Aplicación de insulina. }\end{array}$ & $\begin{array}{l}\text { Médicos y Líderes } \\
\text { Nutrición } \\
\text { Educación Física } \\
\text { Médicos y líderes }\end{array}$ & \\
\hline \multirow{2}{*}{$\begin{array}{l}P \\
R \\
\mathrm{E} \\
\mathrm{T} \\
\mathrm{A} \\
\mathrm{R} \\
\mathrm{D} \\
\mathrm{E}\end{array}$} & $13: 00$ & ALMUERZO & Nutrición & \\
\hline & $\begin{array}{l}13: 30 \\
14: 30 \\
15: 00\end{array}$ & $\begin{array}{l}\text { Velada de despedida. Evaluación } \\
\text { Preparación de la Partida. } \\
\text { Regreso al Hospital de Niños. }\end{array}$ & $\begin{array}{l}\text { Educación Física } \\
\text { Todo el Equipo }\end{array}$ & \\
\hline
\end{tabular}




\section{CAMPAMENTO PARA NIÑOS CON DIABETES 2004 \\ FICHA DE INSCRIPCION}

Apellido y nombres:

Edad:

F M

Nacionalidad: años

Sexo:

Fecha de nacimiento: D.N.I :

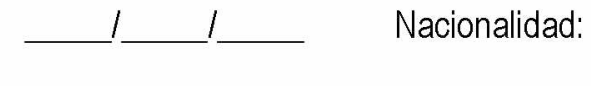
Grado que cursa:

Apellido y nombres del padre:

Apellido y nombres de la madre:

Domicilio: Calle:

$\mathrm{N}^{\circ}$

Ciudad: C.P ( ) Teléfono:

Otro teléfono o celular donde se puede llamar: A quién corresponde:

Antigüedad de la diabetes: años.

¿Cuáles fueron los primeros sintomas de la enfermedad?

Por favor indique si ha padecido episodios de:

Coma

SI NO

SI NO

SI NO

¿Dónde se asiste su diabetes?

Hospital público:

Servicio:

Hospital o Instituto Privado :

DATOS DEL MEDICO QUE LO/LA ATIENDE

Apellido y nombre: Teléfono:

Dirección:

\section{AUTORIZACION}

Autorizo a mi hijo/a. a participar del Campamento para Niños con Diabetes que se llevará a cabo en la localidad de Aguas Verdes del 12/12 al 18/12 del 2004.

Apellido y nombre de quien da la autorización:

DNI:

Domiclio:

Teléfono: 


\section{CAMPAMENTO PARA NIÑOS CON DIABETES 2004 CUESTIONARIO AREA MEDICA}

Apellido y nombres:

Edad: años

Peso: kg Talla: cm. Grupo Sanguíneo:

NO SI

¿Hay otras personas con diabetes en la familia?

¿Quiénes?

¿Practica habitualmente algún deporte o actividad física extraescolar?

$\mathrm{NO} \mathrm{SI}$

¿Cuál?

¿Cuántas veces por semana? 1

$2 \square$

$3 \square$

más de 4

¿Tiene alguna complicación o impedimento que le

limite la práctica de actividad física?

NO SI ¿Cuál/es?

$\square \square$

NO SI ¿Cuál/es?

¿Es alérgico/a a alguna sustancia (alimentos, antibióticos, insulina, etc)?

Aparte de la diabetes, ¿padece de alguna otra NO SI ¿Cuál/es? afección?

Si está tomando algún medicamento, indique cúal o cuáles y por qué:

Fecha aproximada de la última vacuna antitetánica:

1

La preparación de la insulina en la jeringa, ¿la realiza solo? NO SI

La aplicación de la insulina, ¿la realiza solo? NO SI

$\square$

¿En qué sitios se aplica habitualmente la insulina? $\quad$ Brazos $\square \quad$ Abdomen $\square$ Glúteos $\square$ Muslos $\square$

¿Cambia el sitio de aplicación cada vez que se inyecta insulina? 


\section{CAMPAMENTO PARA NIÑOS CON DIABETES 2004 INSULINOTERAPIA}

1. DOSIS DE INSULINA

Marque con una cruz en el casillero correspondiente:

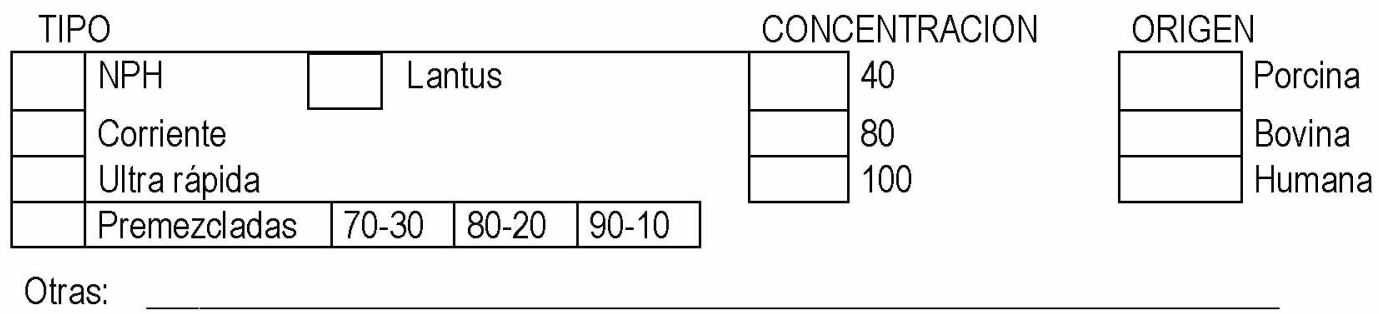

Si utiliza bomba de insulina, indicar programa/s de dosis de insulina (horarios y cantidades):

2. Indicar la cantidad de insulina (dosis en números) que utiliza en el casillero correspondiente:

\begin{tabular}{|l|l|l|l|l|l|l|}
\hline Tiempo del dia & Hora & $\mathrm{NPH}$ & Lantus & Corriente & Ultra rápida & Premezclada \\
\hline Mañana & & & & & & \\
\hline Mediodia & & & & & & \\
\hline Tarde & & & & & & \\
\hline Noche & & & & & & \\
\hline
\end{tabular}

¿Qué tipo de monitoreo realiza?
$\square$ Glucosuria
Cetonuria
$\square$ Glucemia

Si lo realiza, lo hace:

$\square$ Solo $\square$ Con ayuda de otra persona
$\square$ Solo $\square$ Con ayuda de otra persona
$\square$ Solo $\square$ Con ayuda de otra persona

Indicar con una cruz en el casillero que corresponda cuántas veces por día y en qué momento realiza los monitoreos:

\begin{tabular}{|c|c|c|c|}
\hline & & Glucemia & Glucosuria \\
\hline \multirow{2}{*}{ Desayuno } & Antes & & \\
\hline & Después & & \\
\hline \multirow{2}{*}{ Almuerzo } & Antes & & \\
\hline & Después & & \\
\hline \multirow{2}{*}{ Merienda } & Antes & & \\
\hline & Después & & \\
\hline \multirow[b]{2}{*}{ Cena } & Antes & & \\
\hline & Después & & \\
\hline
\end{tabular}

Anote lo que considere de importancia: 


\section{CAMPAMENTO PARA NIÑOS CON DIABETES 2004}

\section{¡IMPORTANTE! \\ LEER CON ATENCION}

Estimados padres,

Queridos amigos:

Les solicitamos, además de completar las otras planillas, que registren en ésta los valores de glucemia y/o glucosuria, unidades de insulina y otras observaciones de la semana anterior al campamento, es decir, la comprendida entre el _ 5/12 _ al _ 11/12 __. Esta planilla la deberán traer al campamento y entregársela al médico en cuanto lleguen. ¡Estos datos son muy importantes para todos nosotros! ¡Gracias por colaborar y por acordarse de traerlos al campamento!

\section{REGISTRO DE DATOS DE LA SEMANA ANTERIOR AL CAMPAMENTO}

\begin{tabular}{|c|c|c|c|c|c|c|c|c|c|c|c|c|c|c|}
\hline \multirow{4}{*}{ Día } & \multicolumn{8}{|c|}{ INSULINA } & \multirow{2}{*}{\multicolumn{5}{|c|}{$\begin{array}{c}\text { MONITOREO } \\
\text { En sangre }\end{array}$}} & \multirow{4}{*}{$\begin{array}{c}\text { Observaciones } \\
\begin{array}{c}\text { Ej.: hipoglucemia } \\
\text { (hora) }\end{array}\end{array}$} \\
\hline & \multirow{2}{*}{\multicolumn{2}{|c|}{$\begin{array}{l}\text { Tipo: } \\
\text { DESAYUNO }\end{array}$}} & \multicolumn{6}{|c|}{ Origen: } & & & & & & \\
\hline & & & ALMU & RZO & MER & ENDA & & & \multirow[t]{2}{*}{$\begin{array}{l}\text { DESA } \\
\text { YUNO }\end{array}$} & \multirow{2}{*}{\begin{tabular}{|l} 
AL \\
MUER \\
ZO \\
\end{tabular}} & \multirow{2}{*}{$\begin{array}{l}\text { ME } \\
\text { RIEN } \\
\text { DA } \\
\end{array}$} & \multirow[t]{2}{*}{ CENA } & \multirow{2}{*}{\begin{tabular}{|l|} 
ANTES \\
DE \\
DORMIR \\
\end{tabular}} & \\
\hline & NPH & Corr & NPH & Corr & NPH & Corr & NPH & Corr & & & & & & \\
\hline \multicolumn{15}{|c|}{ Dom 5} \\
\hline \multicolumn{15}{|l|}{ Lun 6} \\
\hline \multicolumn{15}{|l|}{ Mar 7} \\
\hline \multicolumn{15}{|l|}{ Mie 8} \\
\hline \multicolumn{15}{|l|}{ Jue 9} \\
\hline \multicolumn{15}{|c|}{\begin{tabular}{|l|} 
Vie 10 \\
\end{tabular}} \\
\hline \multicolumn{15}{|l|}{ Sa 11} \\
\hline Dom & & & & & & & & & & & & & & \\
\hline 12 & & & & & & & & & & & & & & \\
\hline
\end{tabular}

\section{IMPORTANTE:}

Hemoglobina glicosilada $\left(\mathrm{HbA}_{1 \mathrm{c}}\right)$ de los primeros días de diciembre de 2004.

Resultado:

Fecha: 


\section{CAMPAMENTO PARA NIÑOS CON DIABETES 2004 CUESTIONARIO AREA NUTRICIONAL}

Apellido y nombres:
Fecha de nacimiento:
Peso actual: $\_\mathrm{kg}$ Talla: $\_$años Sexo: $\square \square$
¿Hay algún alimento que te produzca alergia? $\square$ SI $\square$ ¿Cuál/es?

SI NO

¿Tenés alguna otra enfermedad además de la diabetes? $\square \square$ ¿Cuál/es?

¿Hay algún alimento que tengas prohibido comer? $\square \square$ ¿Cuál/es?

SI NO

¿Hay algún alimento que no te guste? $\square \square$ ¿Cuál/es?

¿Comés golosinas? $\stackrel{\text { SI NO }}{\square \square} \square$ ¿Cuál/es?

¿Cuántas veces por semana?

SI NO

¿Comés pan o galletitas en el almuerzo y la cena?

¿Qué alimentos dietéticos utilizás?

¿Quién realizó tu plan de alimentación?

Nutricionista $\square$ Médico $\square \quad$ Nadie

¿Hace cuánto tiempo? 


\section{CAMPAMENTO PARA NIÑOS CON DIABETES 2004 DATOS DEL PLAN DE ALIMENTACION}

Valor calórico total:
Hidratos de carbono
Proteínas $\%$
Grasas

Indicaciones especiales:

¿Qué comés habitualmente en:

Desayuno

Colación

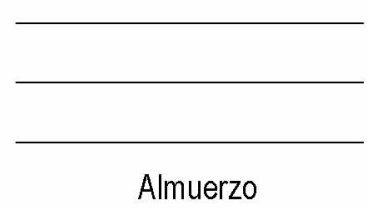

Almuerzo

Colación

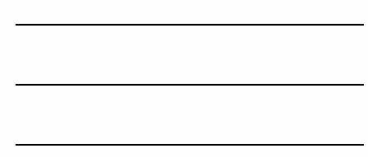

Merienda

Colación
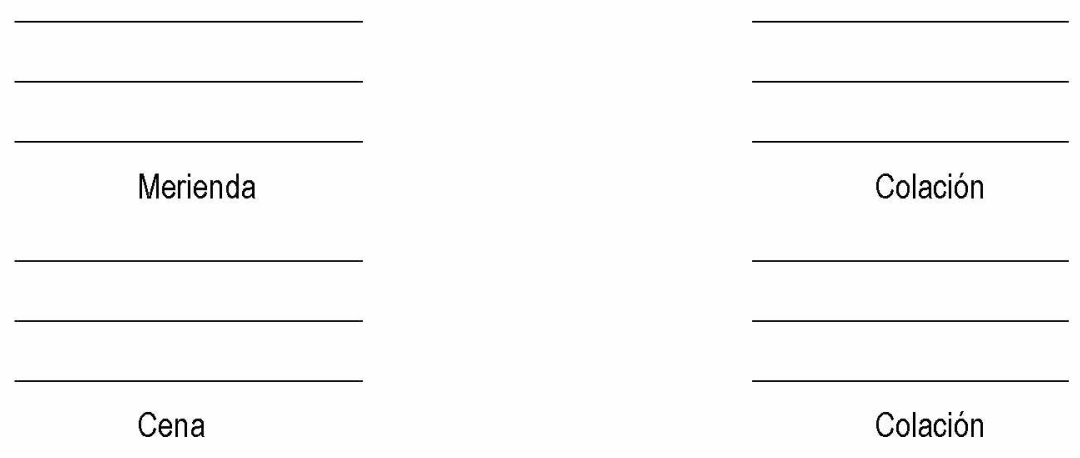

Comentarios especiales: 


\section{ENCUENTRO RECREATIVO Y EDUCATIVO PARA NIÑOS CON DIABETES Y SUS FAMILIARES}

\section{FICHA DE INSCRIPCION}

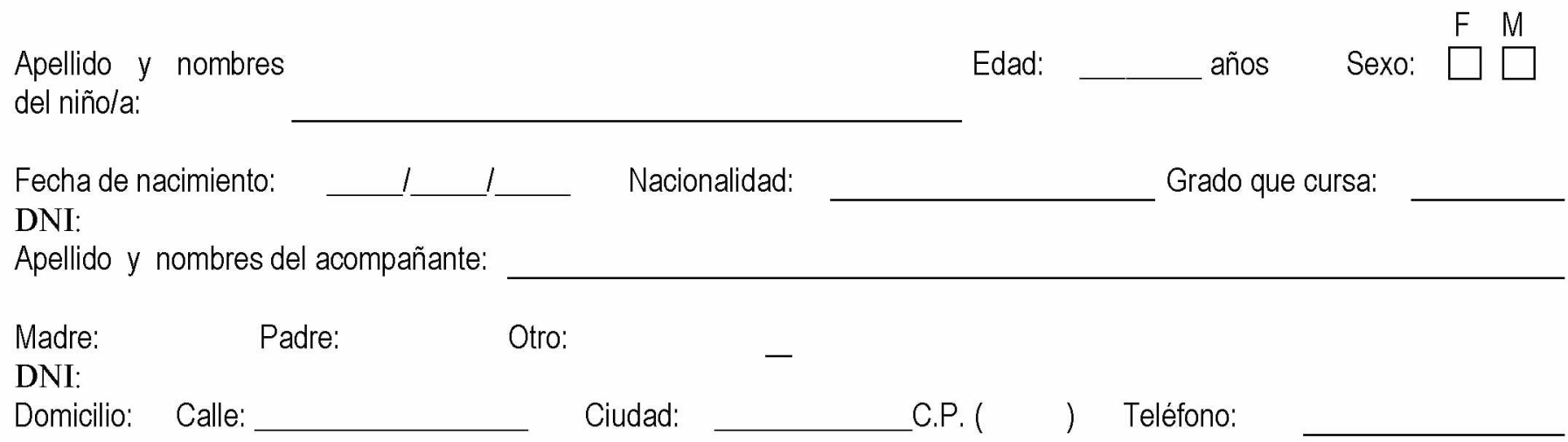

Otro teléfono o celular donde se puede llamar: A quién corresponde:

Antigüedad de la diabetes del niño/a: años.

SI NO SI NO SI NO Por favor indique si ha padecido episodios de (en Coma $\quad \square \square \quad$ Cetoacidosis $\quad \square \square \quad$ Hipoglucemia $\quad \square \square$ los últimos 2 años):

¿Dónde se asiste su diabetes?

Hospital público:

Servicio:

Hospital o Instituto Privado :

\section{DATOS DEL MEDICO QUE LO/LA ATIENDE}

Apellido y nombre: Teléfono:

Dirección:

\section{AUTORIZACION}

Autorizo a mi hijo/a. a participar del III Encuentro Recreativo y Educativo para Niños con diabetes y sus familiares, que se llevará a cabo en la ciudad de La Plata del $16 \quad / \quad 05$ al_ 17 05 acompañado por el

Sr./Sra.

Apellido y nombre de quien da la autorización:

DNI:

Domiclio:

Teléfono: 


\section{ENCUENTRO RECREATIVO Y EDUCATIVO PARA NIÑOS CON DIABETES Y SUS FAMILIARES}

\section{CUESTIONARIO AREA MEDICA}

Apellido y nombres: Edad: años

Peso: $\mathrm{kg}$

Talla: $\mathrm{cm}$.

¿Hay otras personas con diabetes en la familia? $\quad$\begin{tabular}{ll} 
NO & SI \\
\hline & ¿Quiénes?
\end{tabular}

¿Practica habitualmente algún deporte o actividad física extraescolar?

NO SI

¿Cuál?

¿Cuántas veces por semana?

$2 \square$

$3 \square$ más de 4

¿Tiene alguna complicación o impedimento que le NO SI ¿Cuál/es?

limite la práctica de actividad física?

¿Es alérgico/a a alguna sustancia (alimentos, antibióticos, insulina, etc)?

NO SI ¿Cuálles?

Aparte de la diabetes, ¿padece de alguna otra afección?

NO SI ¿Cuál/es?

Si está tomando algún medicamento, indique cúal o cuáles y por qué:

Fecha aproximada de la última vacuna antitetánica: 1

La preparación de la insulina en la jeringa, ¿la realiza solo? NO SI

La aplicación de la insulina, ¿la realiza solo?

NO SI

$\square \square$

¿En qué sitios se aplica habitualmente la insulina? $\quad \operatorname{Brazos} \square \quad$ Abdomen $\square$ Glúteos $\square$ Muslos

¿Cambia el sitio de aplicación cada vez que se inyecta insulina?

NO SI 


\section{ENCUENTRO RECREATIVO Y EDUCATIVO PARA NIÑOS CON DIABETES Y SUS FAMILIARES}

\section{INSULINOTERAPIA}

1. DOSIS DE INSULINA

Marque con una cruz en el casillero correspondiente:

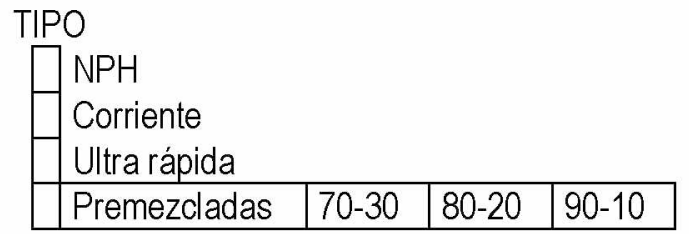

CONCENTRACION
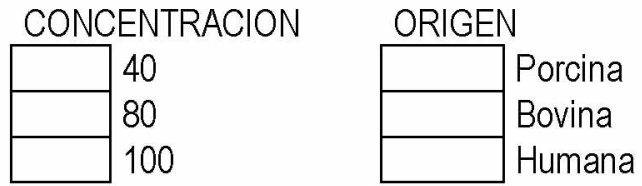

Otr

as'.

Si utiliza bomba de insulina, indicar programa/s de dosis de insulina (horarios y cantidades):

2. Indicar la cantidad de insulina (dosis en números) que utiliza en el casillero correspondiente:

\begin{tabular}{|l|l|l|l|l|l|}
\hline \multicolumn{1}{|c|}{ Tiempo del día } & Hora & $\mathrm{NPH}$ & Corriente & Ultra rápida & Premezclada \\
\hline Mañana & & & & & \\
\hline Mediodía & & & & & \\
\hline Tarde & & & & & \\
\hline Noche & & & & & \\
\hline
\end{tabular}

¿Qué tipo de monitoreo realiza?

Si lo realiza, lo hace:

$\square$ Glucosuria
$\square$ Cetonuria
$\square$ Glucemia

$\square$ Solo

$\square$ Con ayuda de otra persona

Indicar con una cruz en el casillero que corresponda cuántas veces por día y en qué momento realiza los monitoreos:

\begin{tabular}{|c|c|c|c|}
\hline & & Glucemia & Glucosuria \\
\hline \multirow{2}{*}{ Desayuno } & Antes & & \\
\hline & \begin{tabular}{|l|} 
Después \\
\end{tabular} & & \\
\hline \multirow{2}{*}{ Almuerzo } & Antes & & \\
\hline & Después & & \\
\hline \multirow{2}{*}{ Merienda } & Antes & & \\
\hline & \begin{tabular}{|l|} 
Después \\
\end{tabular} & & \\
\hline \multirow{2}{*}{ Cena } & Antes & & \\
\hline & \begin{tabular}{|l|} 
Después \\
\end{tabular} & & \\
\hline
\end{tabular}

Anote lo que considere de importancia: 


\section{ENCUENTRO RECREATIVO Y EDUCATIVO PARA NIÑOS CON DIABETES Y SUS FAMILIARES.}

\section{¡IMPORTANTE!}

\section{LEER CON ATENCION}

Estimados padres,

Queridos amigos:

Les solicitamos, además de completar las otras planillas, que registren en ésta los valores de glucemia y/o glucosuria, unidades de insulina y otras observaciones de la semana anterior al Encuentro, es decir, la comprendida entre el Sábado 9 de Abril al Viernes 15 de Abril. Esta planilla la deberán traer al Encuentro y entregársela al médico en cuanto lleguen. ¡Estos datos son muy importantes para todos nosotros! ¡Gracias por colaborar y por acordarse de traerlos!

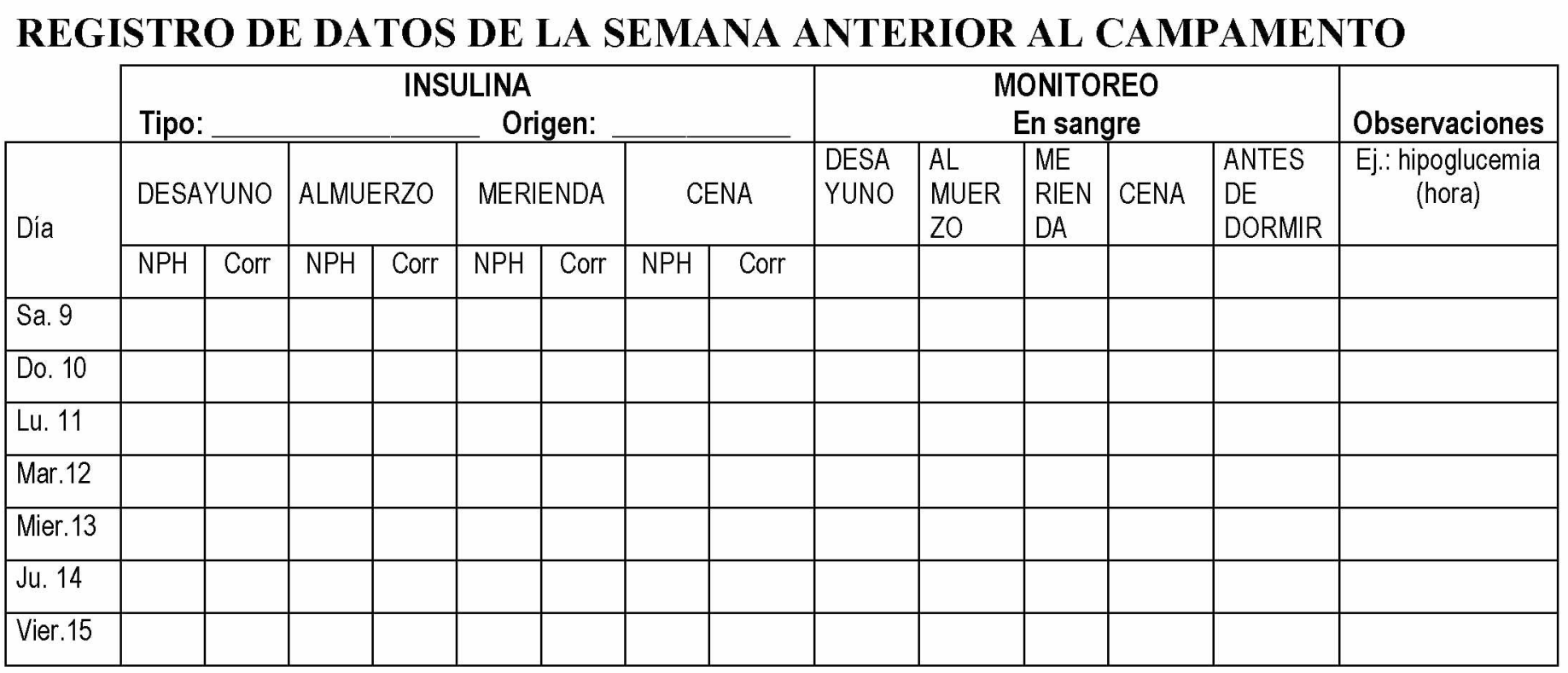

IMPORTANTE:

Hemoglobina glicosilada $\left(\mathrm{HbA}_{1 c}\right)$ del mes de abril del 2005.

Resultado: Fecha: 


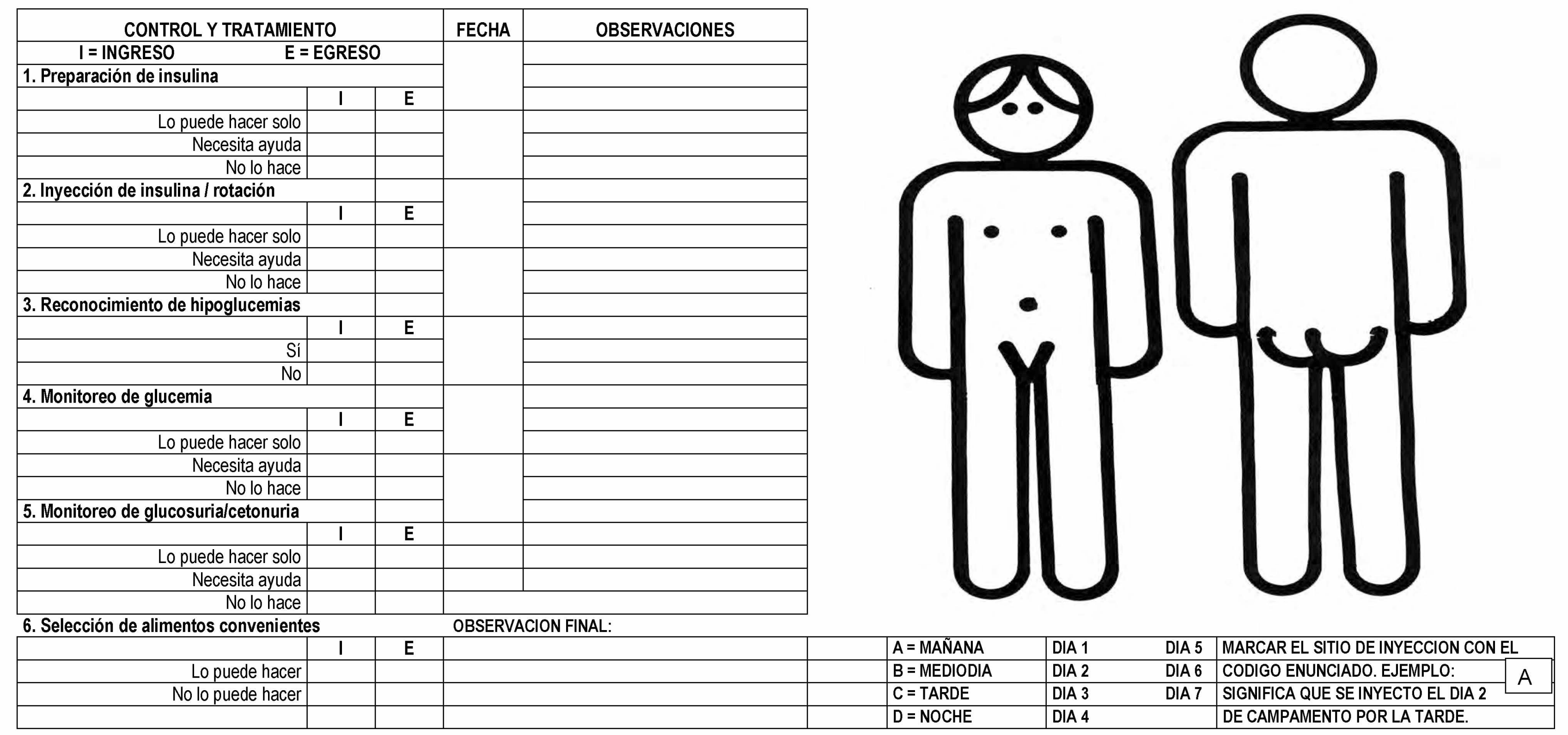


CAMPAMENTO CENEXA 2004

FICHA DE CONTROL DE HABILIDADES

Nombre y Apellido del niño/a:

Nombre y Apellido del evaluador :

\begin{tabular}{|c|c|c|c|c|}
\hline \multicolumn{3}{|c|}{ CONTROL Y TRATAMIENTO } & \multirow[t]{4}{*}{ FECHA } & \multirow[t]{3}{*}{ OBSERVACIONES } \\
\hline I = INGRESO & \multicolumn{2}{|c|}{$E=E G R E S O$} & & \\
\hline \multicolumn{3}{|l|}{ 1. Preparación de insulina } & & \\
\hline & $\mathrm{I}$ & $E$ & & \\
\hline \multicolumn{5}{|l|}{ Lo puede hacer solo } \\
\hline \multicolumn{5}{|l|}{ Necesita ayuda } \\
\hline No lo hace & & & & \\
\hline \multicolumn{5}{|l|}{ 2. Inyección de insulina } \\
\hline & I & $E$ & & \\
\hline \multicolumn{5}{|l|}{ Lo puede hacer solo } \\
\hline \multicolumn{5}{|l|}{ Necesita ayuda } \\
\hline \multirow{2}{*}{\multicolumn{5}{|c|}{$\begin{array}{lr} & \text { No lo hace } \\
\text { 3. Rotación (marque con una } \mathrm{x} \text { ) }\end{array}$}} \\
\hline & & & & \\
\hline & I & $\mathrm{E}$ & & \\
\hline \multicolumn{5}{|l|}{ Brazo der. } \\
\hline \multicolumn{5}{|l|}{ Brazo izq. } \\
\hline \multicolumn{5}{|l|}{ Abdomen lado der. } \\
\hline \multicolumn{5}{|l|}{ Abdomen lado izq. } \\
\hline \multicolumn{5}{|l|}{ Glúteo der. } \\
\hline \multicolumn{5}{|l|}{ Glúteo izq. } \\
\hline \multicolumn{5}{|l|}{ Muslo der. } \\
\hline \multicolumn{5}{|l|}{ Muslo izq. } \\
\hline \multicolumn{5}{|l|}{ 4. Monitoreo de glucemia } \\
\hline & I & $\mathrm{E}$ & & \\
\hline \multicolumn{5}{|l|}{ Lo puede hacer solo } \\
\hline \multicolumn{5}{|l|}{ Necesita ayuda } \\
\hline \multicolumn{5}{|l|}{ No lo hace } \\
\hline \multicolumn{5}{|l|}{ 5. Monitoreo de glucosuria/cetonuria } \\
\hline & I & $\mathrm{E}$ & & \\
\hline \multicolumn{5}{|l|}{ Lo puede hacer solo } \\
\hline \multicolumn{5}{|l|}{ Necesita ayuda } \\
\hline \multicolumn{5}{|l|}{ No lo hace } \\
\hline 6. Selección de alimentos convenientes & & & RACION & \\
\hline & I & $\mathrm{E}$ & & \\
\hline Lo puede hacer & & & & \\
\hline No lo puede hacer & & & & \\
\hline & & & & \\
\hline
\end{tabular}




\section{CAMPAMENTO PARA NIÑOS CON DIABETES 2004}

\section{¡IMPORTANTE! \\ LEER CON ATENCION}

Estimados padres,

Queridos amigos:

Les solicitamos, por favor completar esta planilla con los valores de glucemia y/o glucosuria, unidades de insulina y otras observaciones de la semana posterior al campamento, es decir, la comprendida entre el_19/12____ al___25/12____ Esta planilla la podrán enviar por FAX al CENEXA (0221- 4222081) o al Centro Houssay (0221- 4829375), antes de fin de año. ¡Estos datos son muy importantes para todos nosotros! ¡Gracias por colaborar y por acordarse de enviarla!

REGISTRO DE DATOS DE LA SEMANA POSTERIOR AL CAMPAMENTO

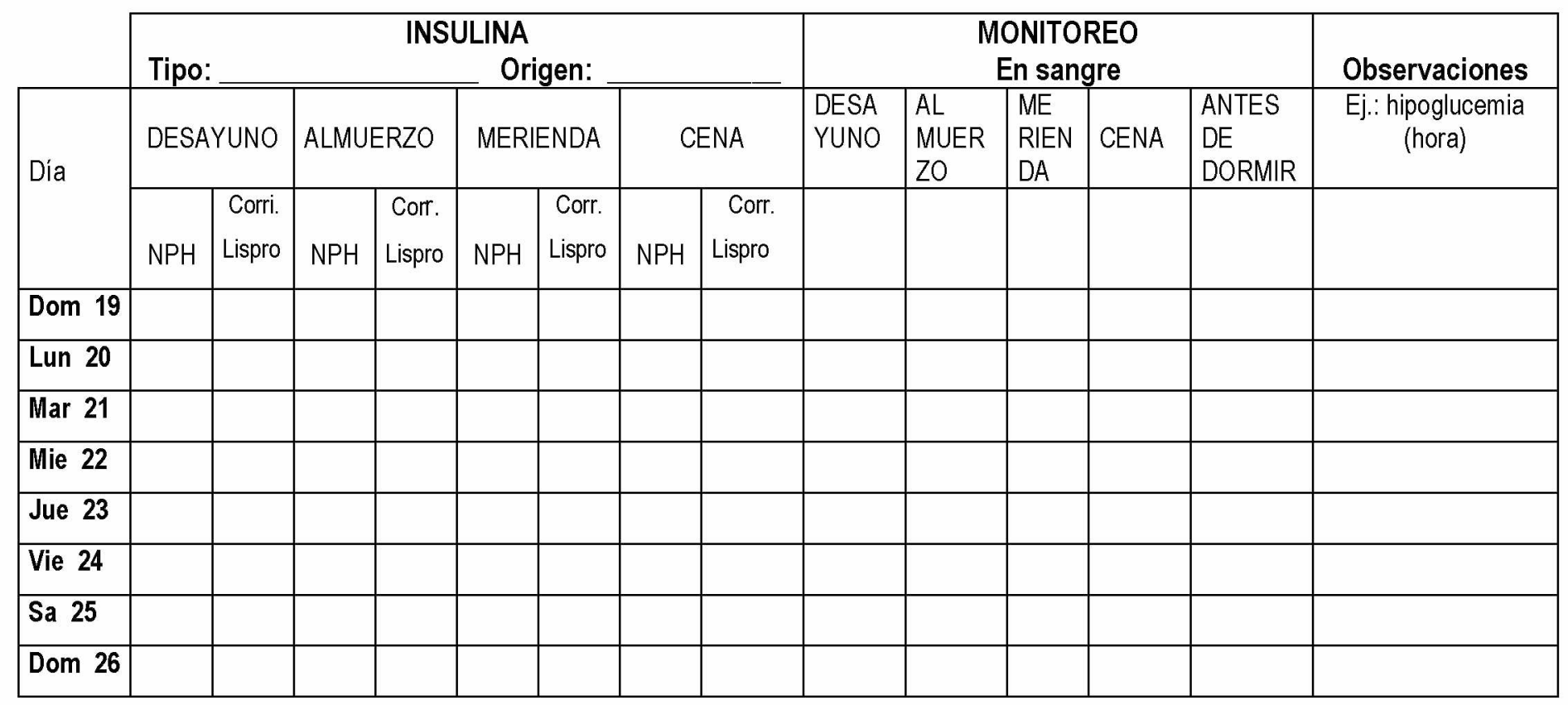

\section{IMPORTANTE:}

Por favor complete con letra clara y no pierda la planilla hasta que sepa que la hemos recibido! Muchas Gracias! 


\section{CAMPAMENTO 2004}

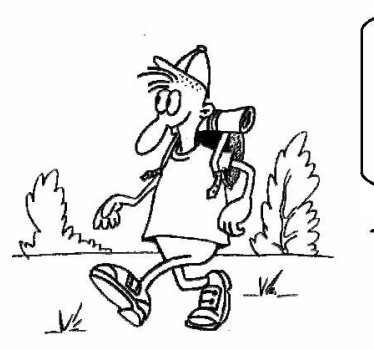

Me gustaría saber algo sobre Diabetes ¿Podrías contestarme estas preguntas?

\section{Marcá con una $X$ la respuesta que crees correcta}

1) ¿Cuáles son los valores normales de glucemia en ayunas?

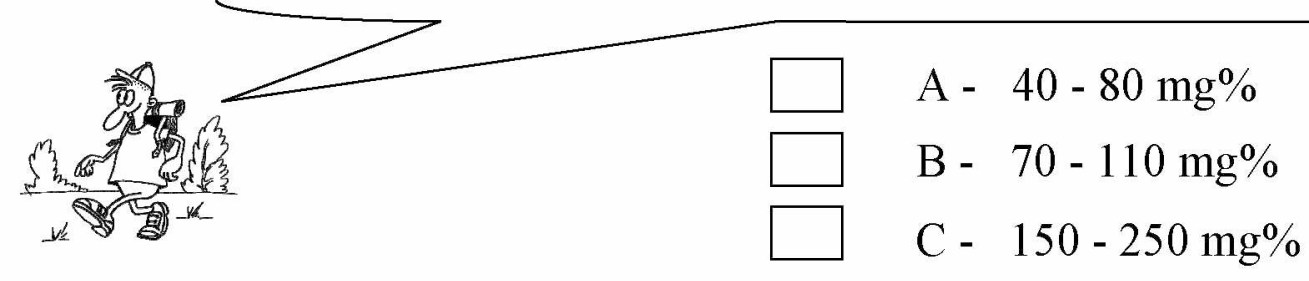

2) Si en el almuerzo un chico/a con diabetes tiene poco hambre, ¿Qué es lo que le convendría comer primero?
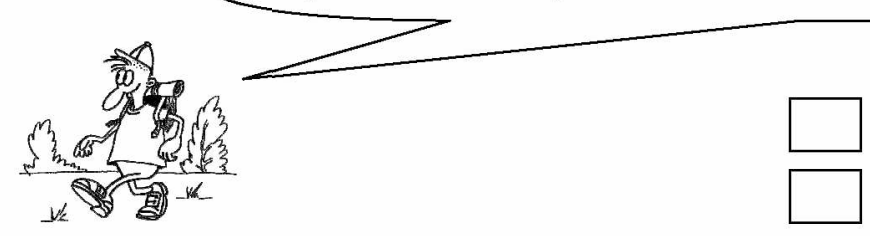

A - carnes

B - verduras

$\square$

C - fideos

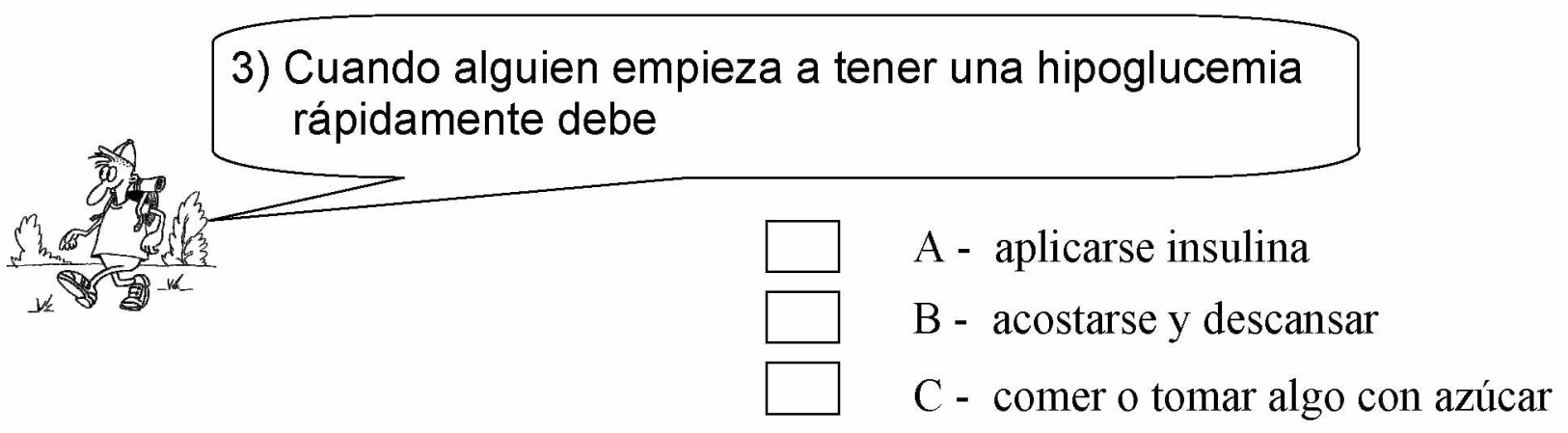

4) Si uno se equivoca al hacer la mezcla de insulina tiene que

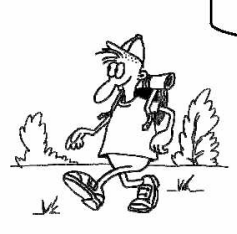

A - tirarla

$\square$

B - inyectársela igual

$\square$

C - agregar más insulina 
5) Cuando se prepara la mezcla de insulina

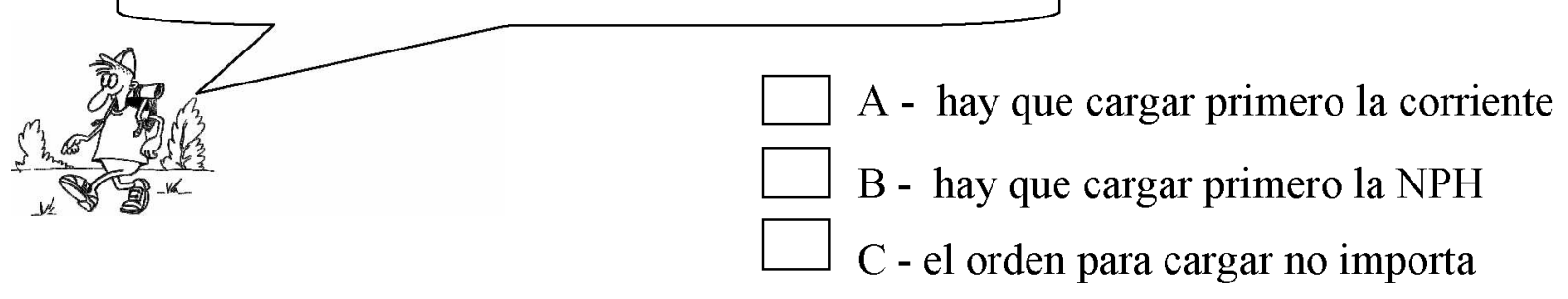

6) Al salir a correr, el lugar en que no debe inyectarse la insulina es
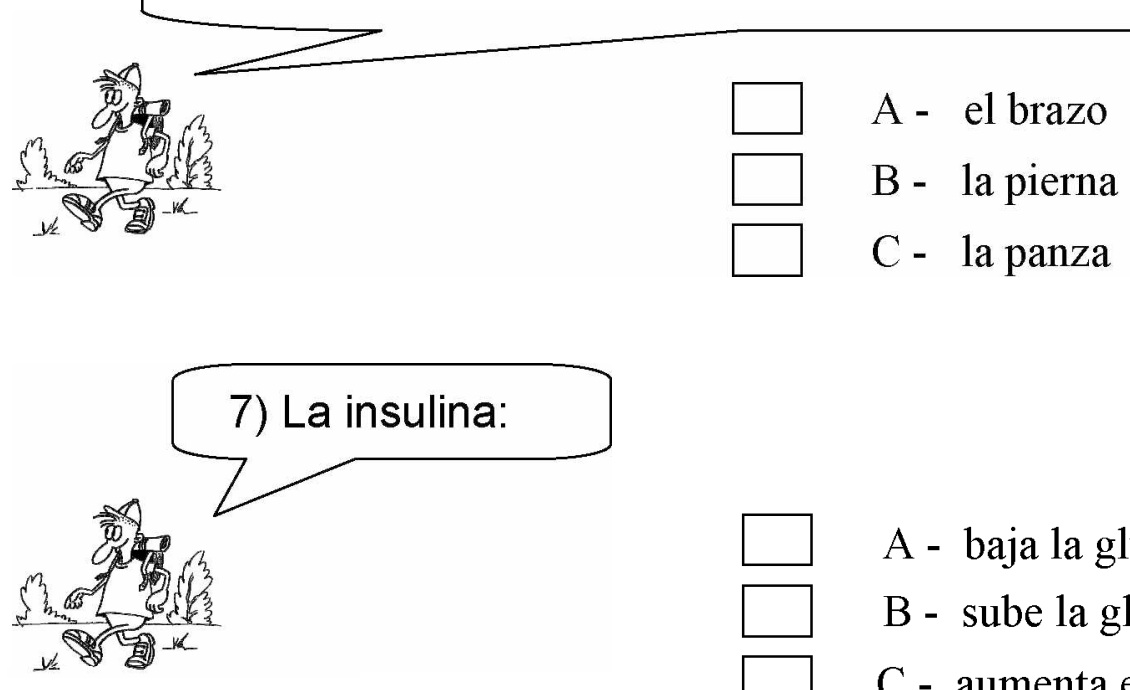

A - baja la glucemia

B - sube la glucemia

C - aumenta el azúcar en la orina

8) Hacer mucho ejercicio después de inyectarse la insulina sin comer hidratos de carbono

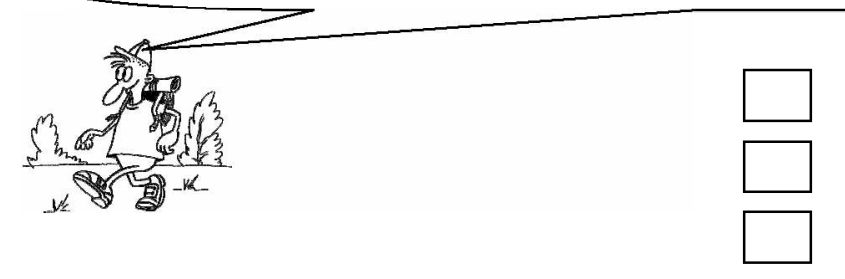
A - puede dar una hipoglucemia
B - pude subir la glucemia
C - no produce ningún efecto

9) ¿Cuál de estos alimentos tienen hidratos de carbono y suben la glucemia?

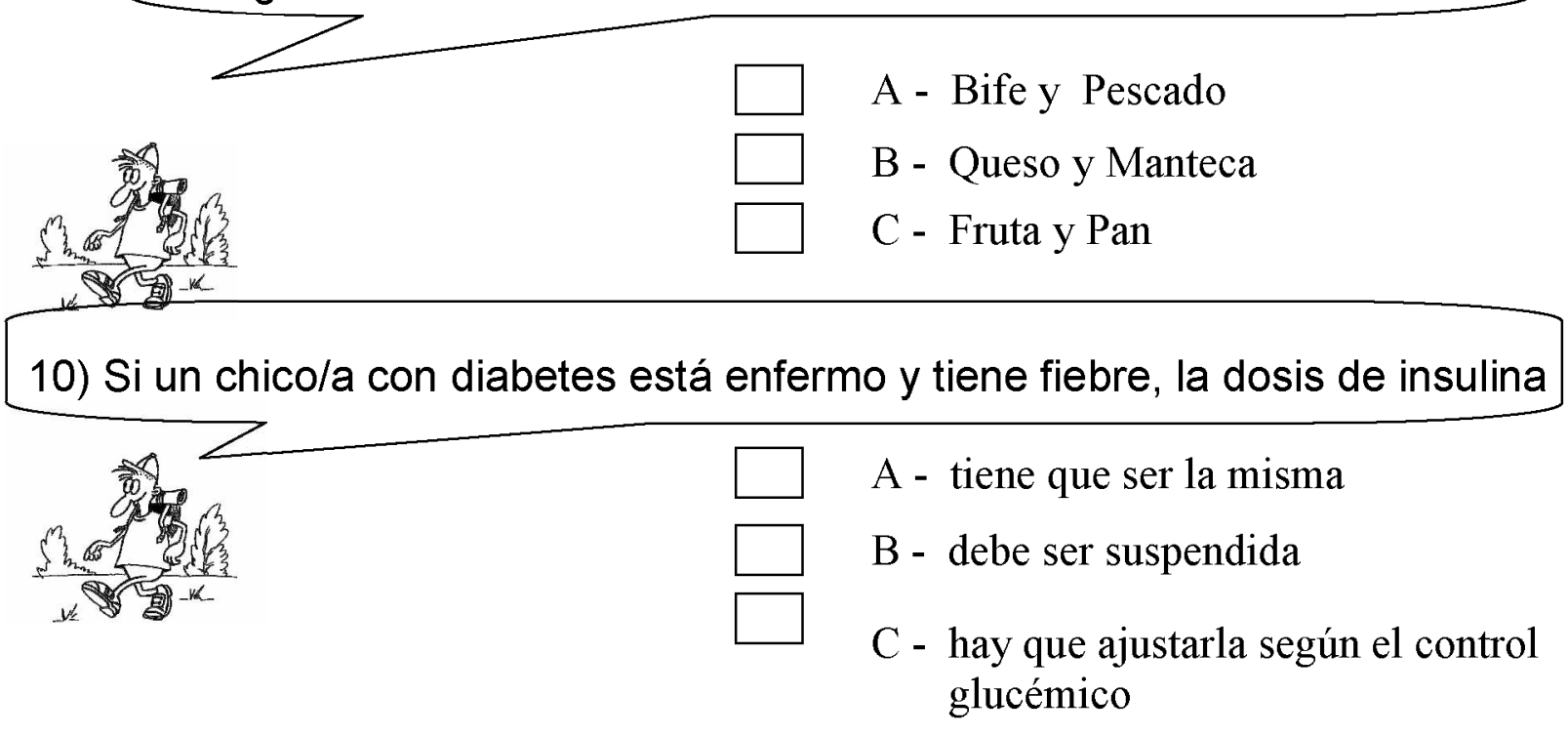




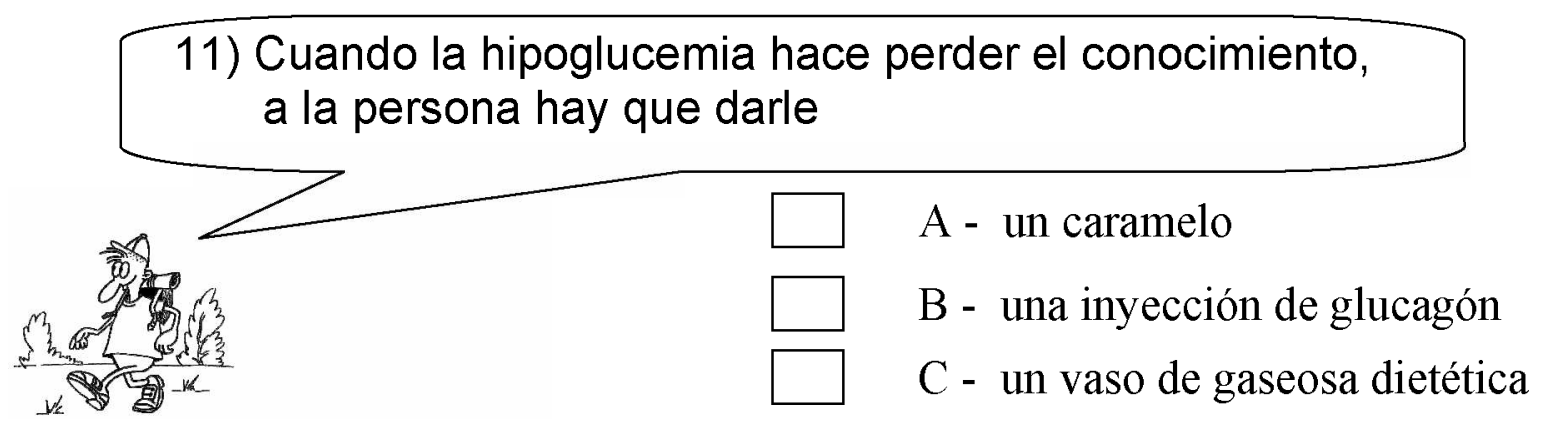

12) Un chico/a con diabetes debe llevar siempre consigo
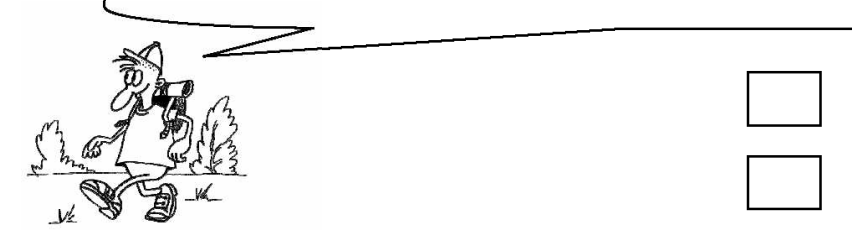

A - caramelos

$\square$ B - aspirinas

$\square$ C - una fruta

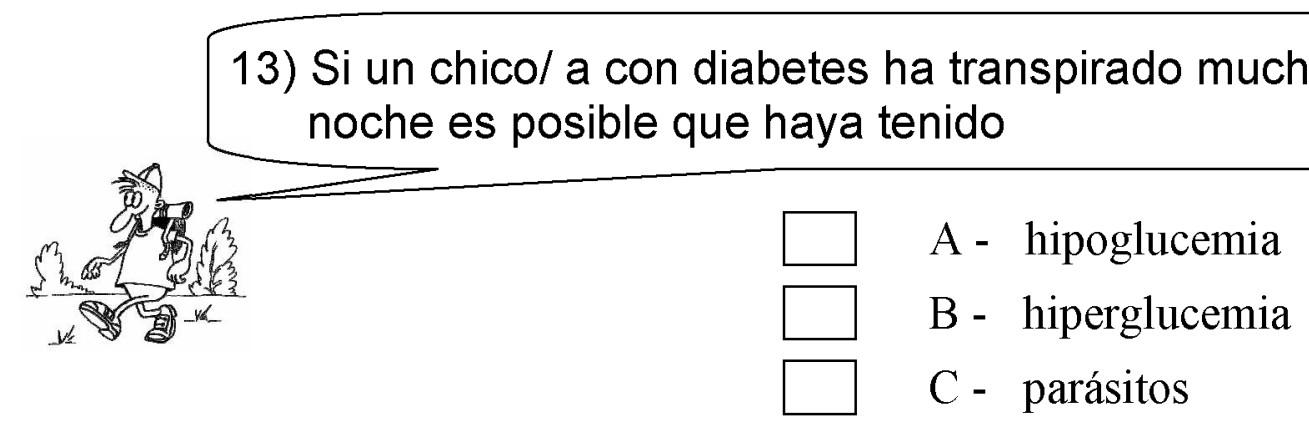

14) Tener mucha sed y orinar muchas veces son síntomas de
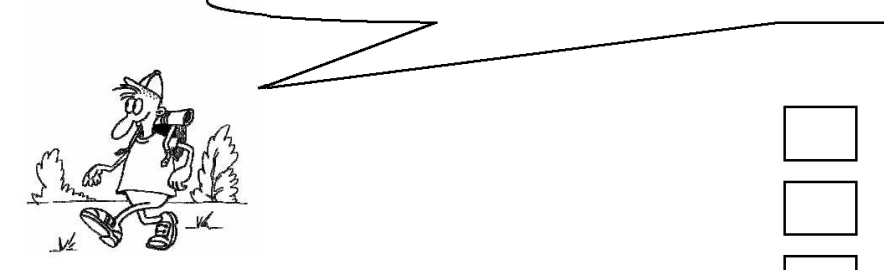

A - hipoglucemia

B - hiperglucemia

$\square$

C - glucemia normal

15) Es necesario controlar la glucemia

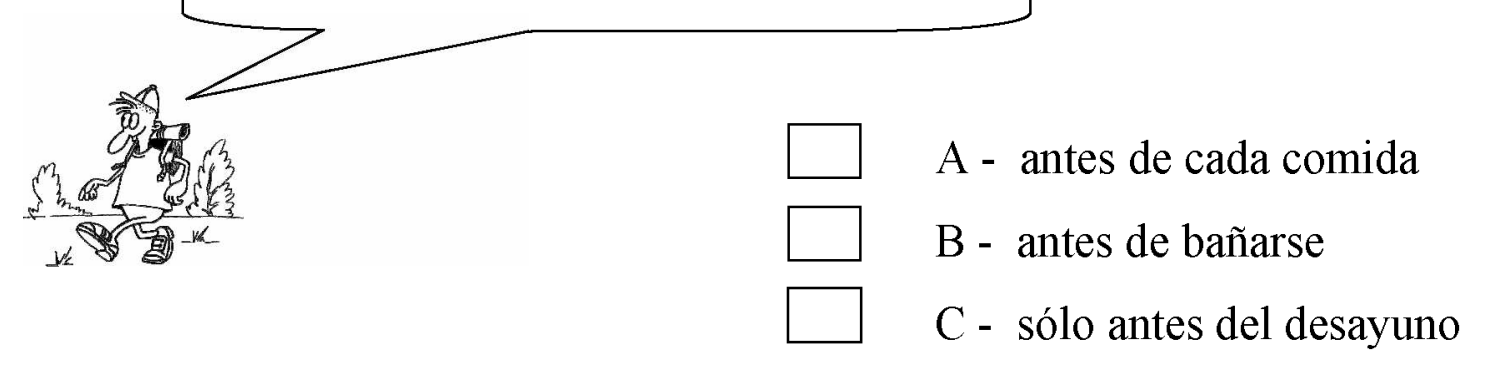



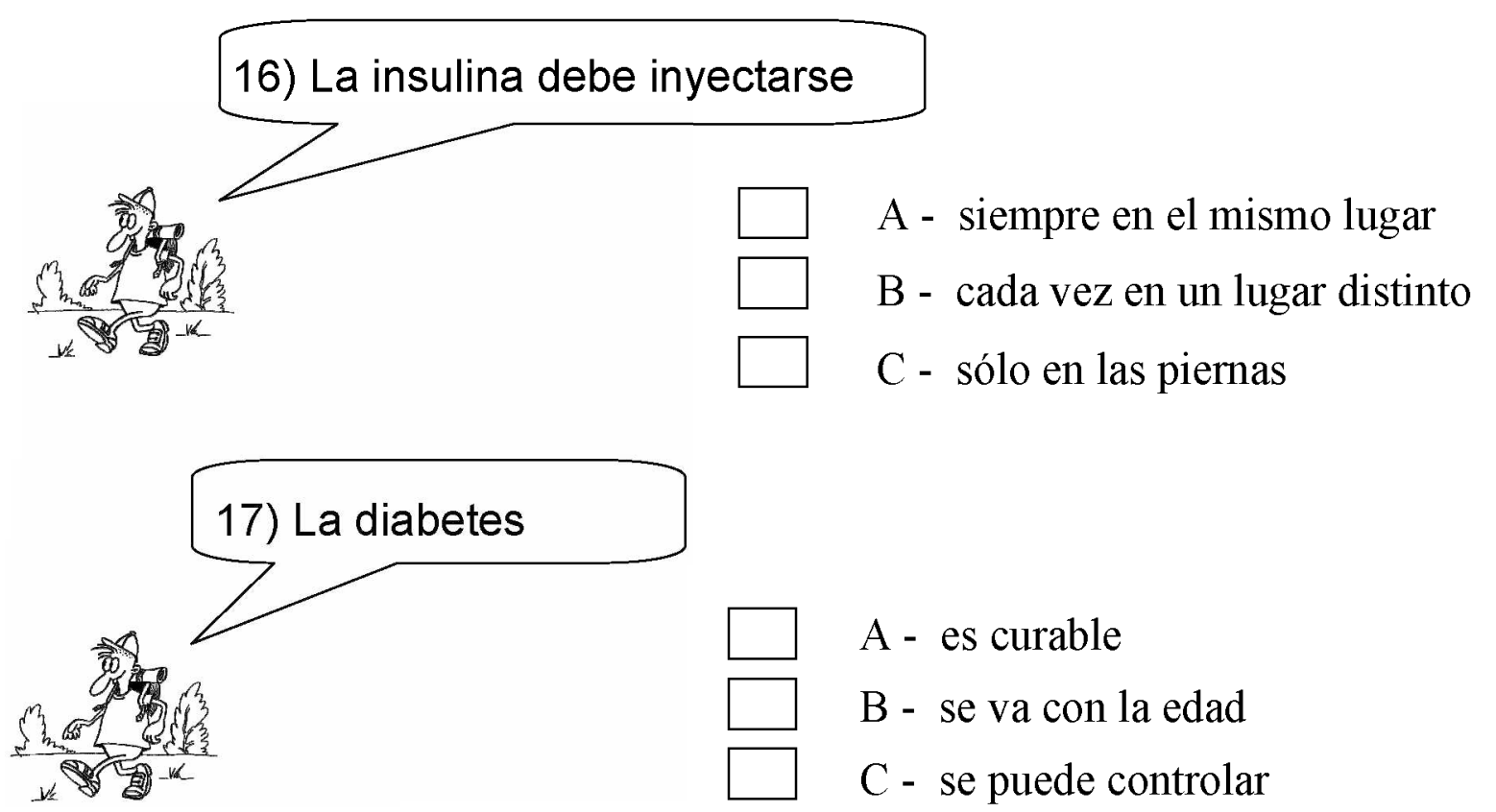
$\square$ A - es curable
$\square$
B - se va con la edad
C - se puede controlar
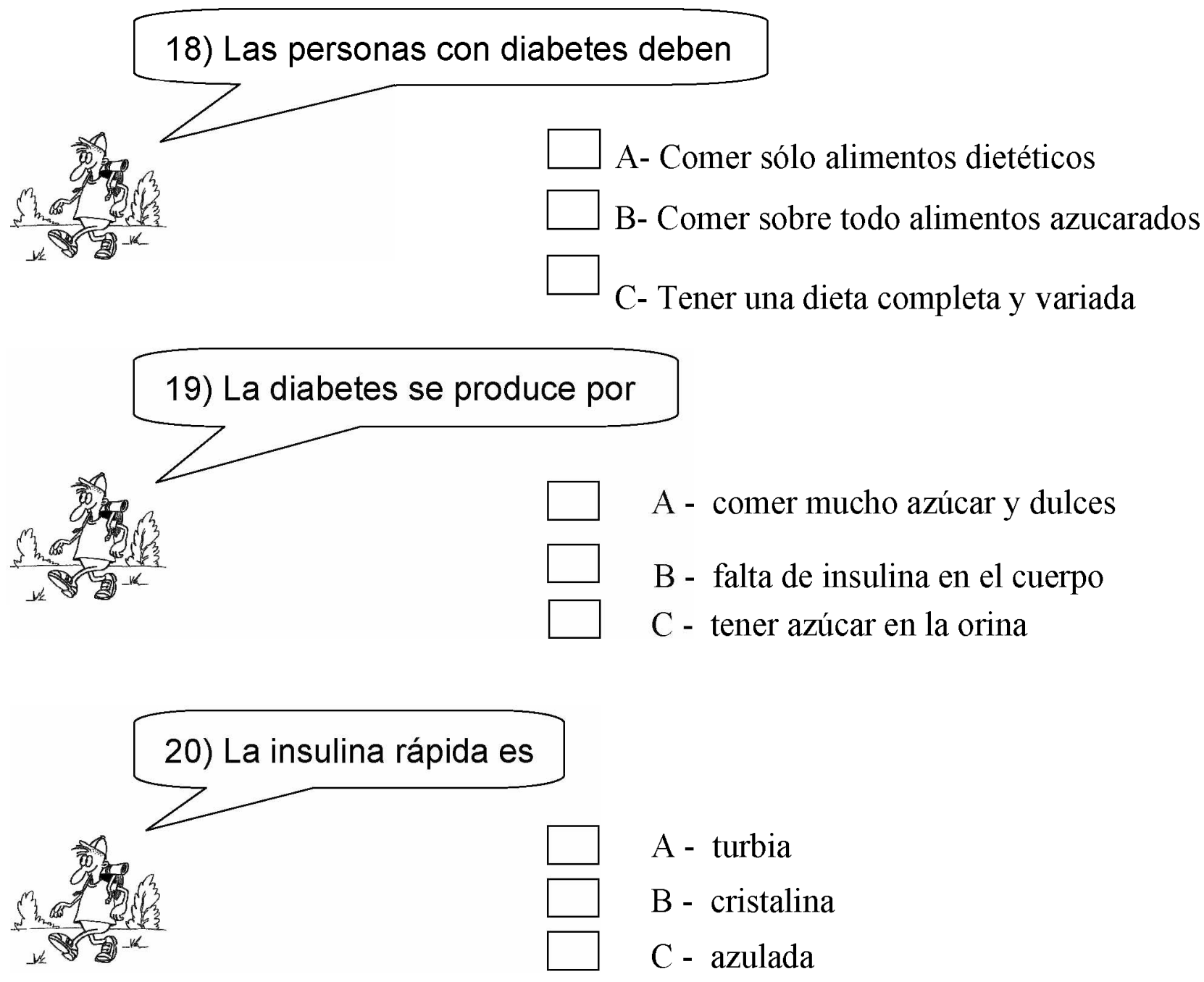

Fecha inicio: / / Fecha final: / / 


\section{CAMPAMENTO PARA NIÑOS CON DIABETES}

LUGAR: Centro Recreativo San Luis Fragata Sarmiento 450- Aguas Verdes

\section{FECHA: 12 al 18 de diciembre de 2004}

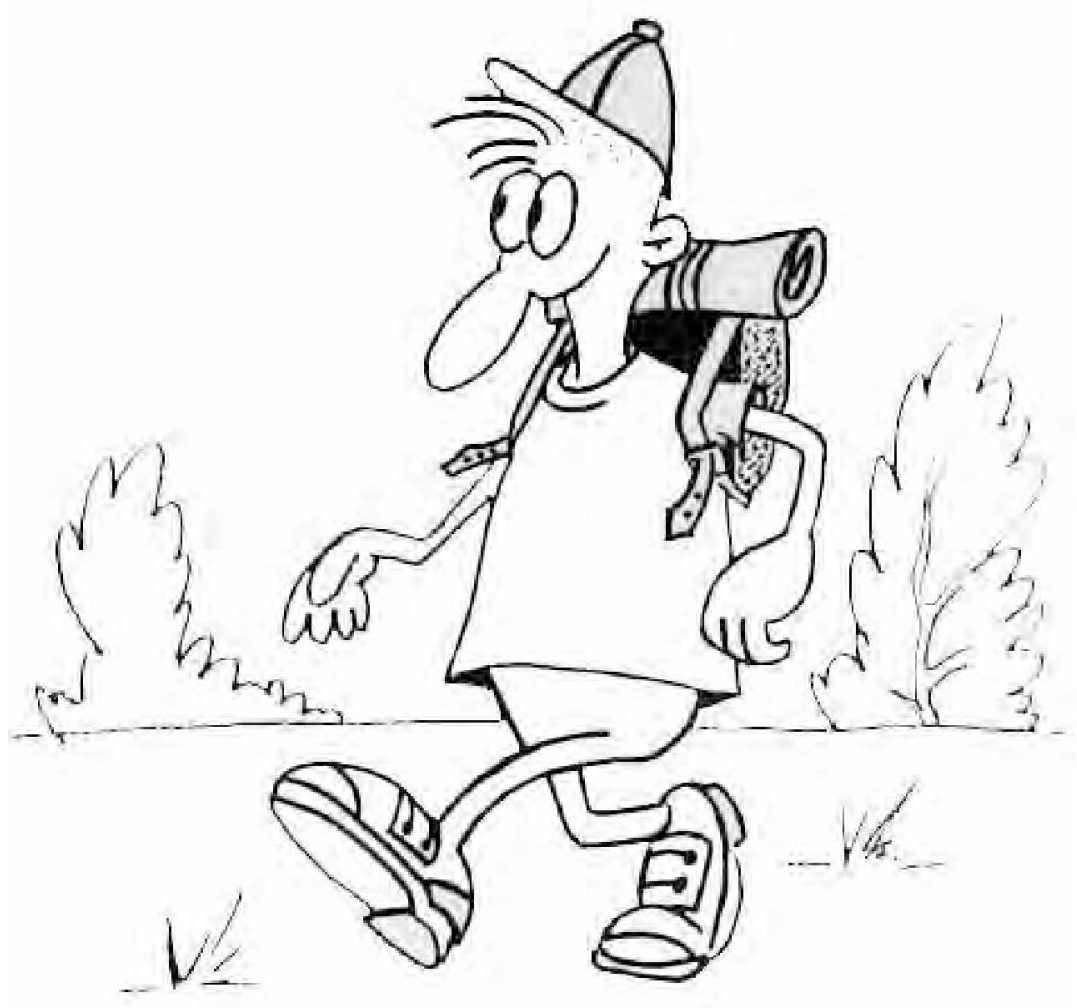

INSCRIPCIÓN E INFORMES:

- CENEXA - Centro de Endocrinología Experimental y Aplicada, Facultad de Ciencias Médicas, calle 60 y 120. Teléfono: 423-6712 Fax: 422-2081

- Servicio de Endocrinología del Hospital de Niños "Sor María Ludovica", calle 14 entre 65 y 66 . Teléfono: $453-5901$

CONVENIOS:

CENEXA - Dirección de Cultura y Educación de la Prov. de Buenos Aires CIC-Comisión de Investigaciones Científicas y Técnicas de la Provincia de Buenos Aires.

COLABORA:

Unidad de Endocrinología Hospital de Niños "Sor María Ludovica" 
CAMPAMENTO 2004

12 al 18 de diciembre CENTRO RECREATIVO SAN LUIS, AGUAS VERDES

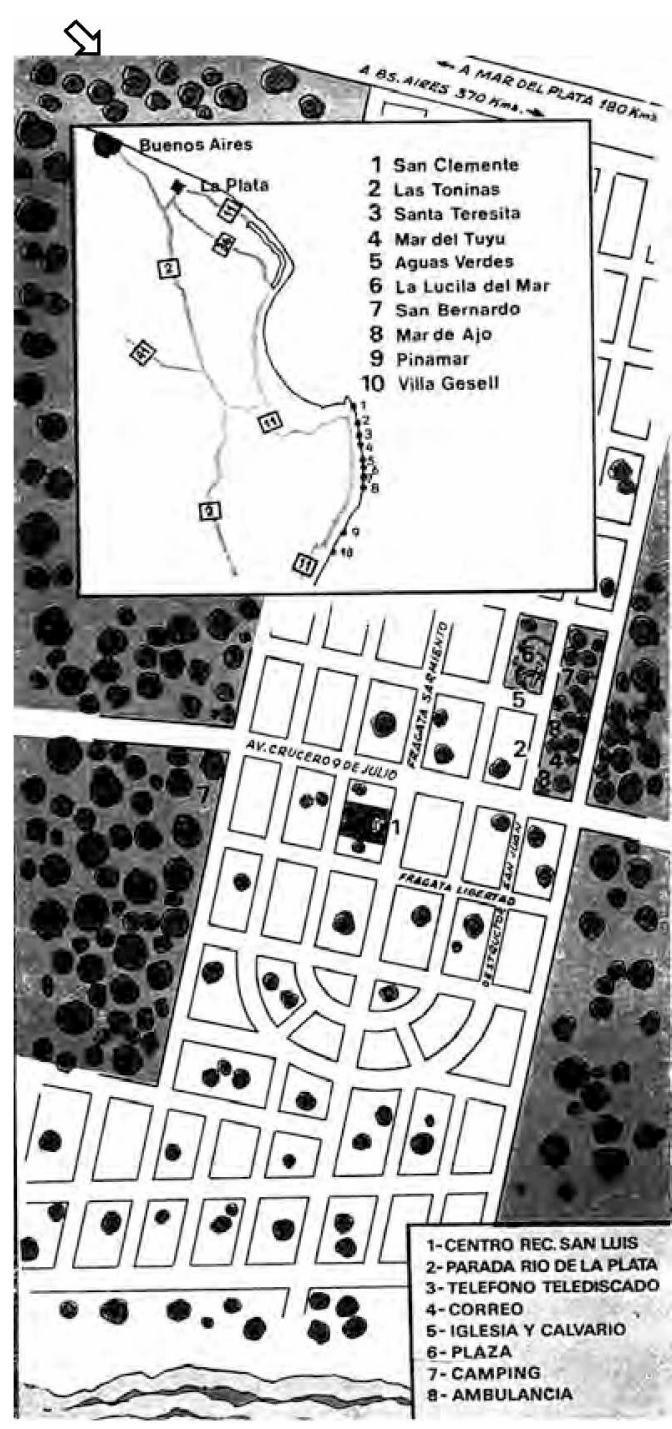

EQUIPO MULTIDISCIPLINARIO RESPONSABLE DEL CAMPAMENTO

Coordinadora General: Prof. Nora Mercuri

Médicos diabetólogos:

Dra. Irma Moreno

Dra. Viviana Balbi

Asistente de médicos: Liliana Rizzuti

Nutricionistas:

Lic. Gladys Vairetta

Prof. de Educ. Física: Prof. Nora Mercuri Prof. Viviana Arrechea

Líderes:

Ariel Decicilia

Daniel Gómez Oscoz

Soledad López

Josefina Marcuzzi

María Isabel Morgner

Hernán Salaberry

Mariela Tunisse

INFORMES E INSCRIPCION:

CENEXA, Facultad de Ciencias Médicas

Calles 60 y 120 - 1900 La Plata

T.E.: (0221) 423-6712

Horario: 9.30 a 16.00 hs.

\section{CAMPAMENTO PARA NIÑOS CON DIABETES}

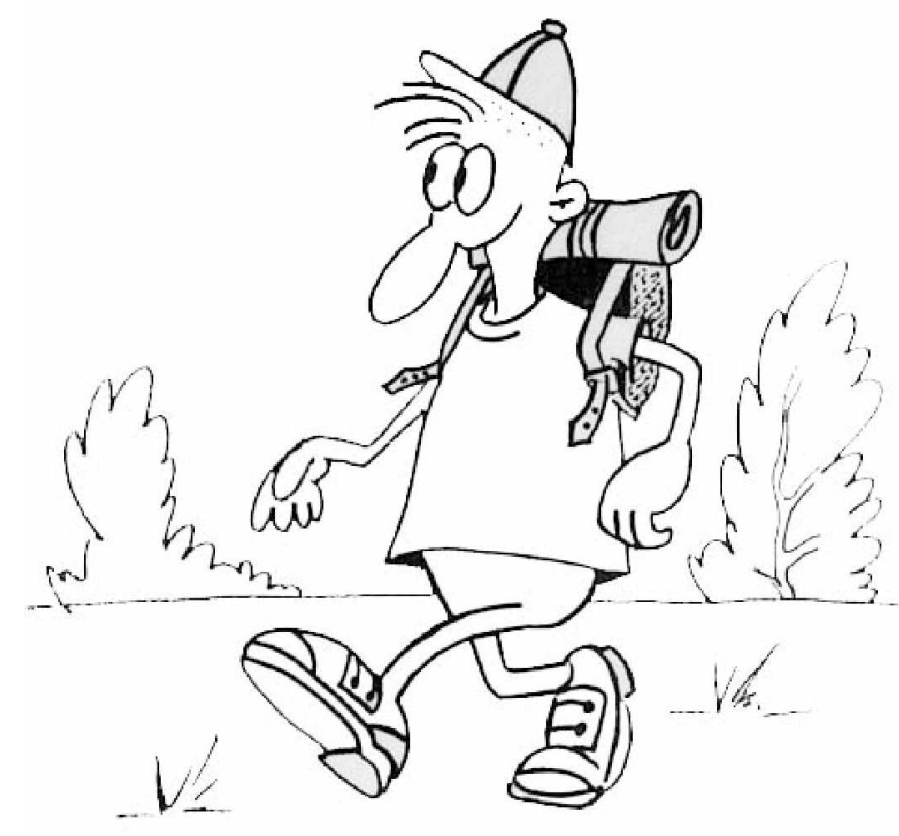

CENEXA - Centro de Endocrinología Experimental y Aplicada (UNLP-CONICET-Centro Colaborador de la OPS/OMS) Ministerio de Salud de la Provincia de Buenos Aires Dirección General de Cultura y Educación, Provincia de Buenos Aires

Colabora:

Unidad de Endocrinología, Hospital de Niños "Sor María Ludovica" 
Los Campamentos para niños con diabetes han adquirido una importancia creciente en el ámbito internacional, porque representan una experiencia de vida en donde las posibilidades de enseñanza-aprendizaje se presentan como ideales, ya que se desarrollan en un ambiente distendido y ampliamente aceptado por los niños.

Las vivencias del Campamento ayudan al niño a desarrollar independencia, autoconfianza y una actitud responsable y participativa dentro de su tratamiento, conociéndose, valiéndose y valorándose a sí mismo y al grupo humano que lo rodea.

En un clima de diversión y colaboración mutua, el niño aprende a familiarizarse con los componentes de su tratamiento específico, incorporando hábitos adecuados de alimentación, de actividad física, manejando métodos de autocontrol y aplicación de insulina.

El programa de Campamento abarca desde actividades atlético-deportivas, recreativas, estético-expresivas, de campamentismo, hasta juegos y charlas de educación diabetológica.

La organización y el desarrollo de nuestro Campamento está a cargo de un equipo de trabajo multidisciplinario integrado por diabetólogos, nutricionistas, profesores de educación física y jóvenes líderes, todos ellos con experiencia en el control y manejo de pacientes con diabetes $y$ en campamentos de este tipo.

\section{UN DIA DE CAMPAMENTO}

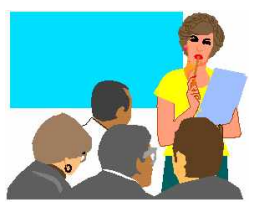

Educación diabetológica

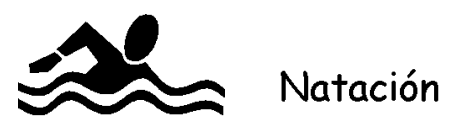

(juegos, charlas)
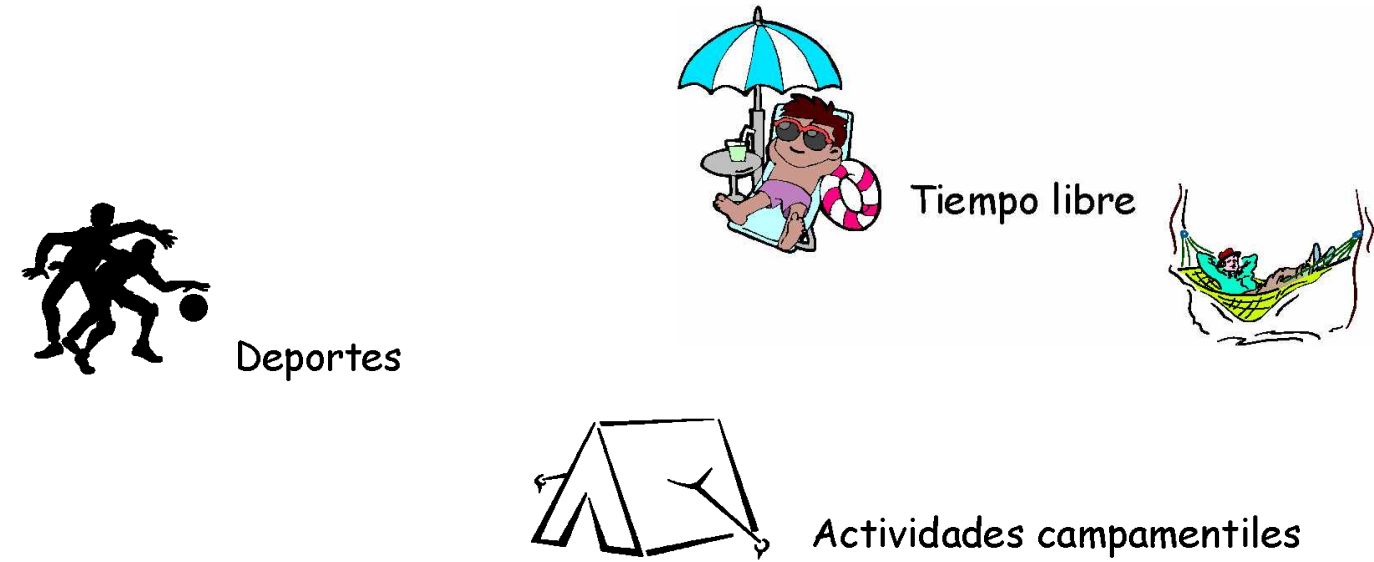

Actividades campamentiles

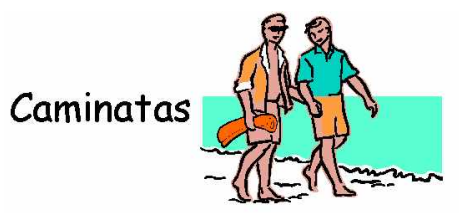

Actividades musicales

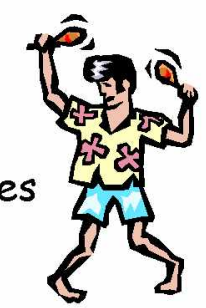

Actividades estético-expresivas

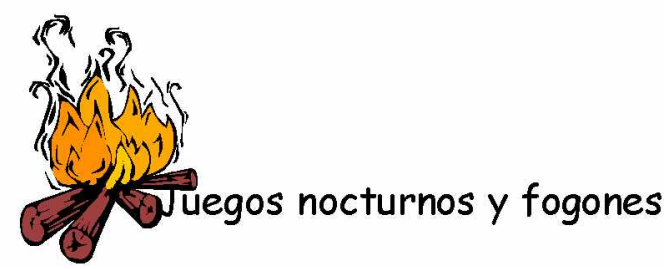




\section{Agradezco:}

- Al Dr. Juan José Gagliardino por su dirección y permanente apoyo y por la confianza depositada todos estos años para desarrollar en nombre del CENEXA los campamentos para niños con diabetes.

- A mi familia por su comprensión y tolerancia.

- A las Dras. Irma Moreno y Viviana Balbi y a todo el maravilloso equipo interdisciplinario que me acompaña año tras año en la organización y desarrollo de los campamentos y encuentros familiares.

- A los jóvenes líderes del campamento, que con su ejemplo y guía ayudan a los niños a proyectarse confiadamente al futuro.

- A mis compañeros de trabajo Joaquín Caporale, Patricia Oliver, Fernando Siri y Guillermina Pfirter por su permanente colaboración y apoyo.

- A María Inés Urrutia por la realización del análisis estadístico de los datos del presente estudio.

- A Elma Perez de Gagliardino y Alberto Natale por su colaboración en el diseño de la presentación.

- A los niños y familiares participantes, por su confianza e interés incondicional en participar de este proyecto.

A las siguientes instituciones y empresas que contribuyeron en forma desinteresada con el Campamento 2004 y el Encuentro Familiar 2005:

- Abbott Laboratories Argentina S.A.

- Aventis Pharma S.A.

- Bayer Argentina S.A.

- Eli Lilly Interamérica, Inc.

- GLAXO SMITH KLINE. 
- JANSSEN CILAG

- Johnson \& Johnson Medical S.A.

- Laboratorios BETA

- MERISANT ARGENTINA

- NOVO NORDISK PHARMA ARGENTINA S.A.

- Productos ROCHE

- Roemmers SAICF

- U.P.C.N Unión Personal Civil de la Nación -La Plata- 